\title{
Introduction to Topological Groups
}

\author{
Dikran Dikranjan \\ To the memory of Ivan Prodanov
}

\begin{abstract}
These notes provide a brief introduction to topological groups with a special emphasis on Pontryaginvan Kampen's duality theorem for locally compact abelian groups. We give a completely self-contained elementary proof of the theorem following the line from [36]. According to the classical tradition, the structure theory of the locally compact abelian groups is built parallelly.
\end{abstract}

\section{Introduction}

Let $\mathcal{L}$ denote the category of locally compact abelian groups and continuous homomorphisms and let $\mathbb{T}=\mathbb{R} / \mathbb{Z}$ be the unit circle group. For $G \in \mathcal{L}$ denote by $\widehat{G}$ the group of continuous homomorphisms (characters) $G \rightarrow \mathbb{T}$ equipped with the compact-open topology ${ }^{1}$. Then the assignment

$$
G \mapsto \widehat{G}
$$

is a contravariant endofunctor $\widehat{\imath}: \mathcal{L} \rightarrow \mathcal{L}$. The celebrated Pontryagin-van Kampen duality theorem ([82]) says that this functor is, up to natural equivalence, an involution i.e., $\widehat{\widehat{G}} \cong G$ (see Theorem 7.36 for more detail). Moreover, this functor sends compact groups to discrete ones and viceversa, i.e., it defines a duality between the subcategory $\mathcal{C}$ of compact abelian groups and the subcategory $\mathcal{D}$ of discrete abelian groups. This allows for a very efficient and fruitful tool for the study of compact abelian groups, reducing all problems to the related problems in the category of discrete groups. The reader is advised to give a look at the Mackey's beautiful survey [75] for the connection of charactres and Pontryagin-van Kampen duality to number theory, physics and elsewhere. This duality inspired a huge amount of related research also in category theory, a brief comment on a specific categorical aspect (uniqueness and representability) can be found in $\S 8.1$ of the Appendix.

The aim of these notes is to provide a self-contained proof of this remarkable duality theorem, providing all necessary steps, including basic background on topological groups and the structure theory of locally compact abelian groups. Peter-Weyl's theorem asserting that the continuous characters of the compact abelian groups separate the points of the groups (see Theorem 6.4) is certainly the most important tool in proving the duality theorem. The usual proof of Peter-Weyl's theorem involves Haar integration in order to produce sufficiently many finite-dimensional unitary representations. In the case of abelian groups the irreducible ones turn out the be one-dimensional. i.e., charactres. We prefer here a different approach. Namely, Peter-Weyl's theorem can be obtained as an immediate corollary of a theorem of Følner (Theorem 5.12) whose elementary proof uses nothing beyond elementary properties of the finite abelian groups, a local version of the Stone-Weierstraß approximation theorem proved in $\S 2$ and the Stone-Čech compactification of discrete spaces. As another application of Følner's theorem we describe the precompact groups (i.e., the subgroups of the compact groups) as having a topology generated by continuous characters. As a third application of Følner's theorem one can obtain the existence of the Haar integral on locally compact abelian groups for free (see [36, §2.4, Theorem 2.4.5]).

The notes are organized as follows. In Section 2 we recall basic results and notions on abelian groups and general topology, which will be used in the rest of the paper. Section 3 contains background on topological groups, starting from scratch. Various ways of introducing a group topology are considered ( $(3.2)$, of which the prominent one is by means of characters (§3.2.3). In $\S 3.6$ we recall the construction of Protasov and Zelenyuk [88] of topologies arising from a given sequence that is required to be convergent to 0. Connectedness and related properties in topological groups are discussed in $§ 3.5$. In $\S 3.7$ the Markov's problems on the existence of non-discrete Hausdorff group topologies is discussed. In $\$ 3.7 .1$ we introduce two topologies, the Markov topology and the Zariski topology, that allow for an easier understanding of Markov's problems. In $\S 3.7 .2$ we

\footnotetext{
${ }^{1}$ having as a base of the neighborhoods of 0 the family of sets $W(K, U)=\{\chi \in \widehat{G}: \chi(K) \subseteq U\}$, where $K \subseteq G$ is compact and $U$ is an open neighborhood of 0 in $\mathbb{T}$.
} 
describe the Markov topology of the infinite permutation groups, while $\S 7.3 .3$ contains the first two examples of non-topologizable groups, given by Shelah and Ol'shanskii, respectively. The problems arising in extension of group topologies are the topic of $\S 7.3 .4$. Several cardinal invariants (weight, character and density character) are introduced in $\S 3.8$, whereas $\S 3.9$ discuses completeness and completions. Further general information on topological groups can be found in the monographs or surveys [3, 26, 27, 28, 36, 70, 79, 82].

Section 4 is dedicated to specific properties of the (locally) compact groups used essentially in these notes. The most important property we recall in $\S 4.1$ is the open mapping theorem In $\S 4.2$ we give an internal description of the precompact groups using the notion of a big (large) set of the group. In $\S 4.3$ we recall (with complete proofs) the structure of the closed subgroups of $\mathbb{R}^{n}$ as well as the description of the closure of an arbitrary subgroup of $\mathbb{R}^{n}$. These groups play an important role in the whole theory of locally compact abelian groups. In $\S 5$ we expose the proof of Følner's theorem (see Theorem 5.12). The proof, follows the line of [36]. An important ingredient of the proof is also the crucial idea, due to Prodanov, that consists in elimination of all discontinuous characters in the uniform approximation of continuous functions via linear combinations of characters obtained by means of Stone-Weierstraß approximation theorem (Prodanov's lemma 5.10).

In Section 6 we give various applications of Følner's theorem. The main one is an immediate proof of PeterWeyl's theorem. In this chapter we give also several other applications of Følner's theorem and Peter-Weyl's theorem: a description of the precompact group topologies of the abelian groups (§6.1) and the structure of the compactly generated locally compact abelian groups ( $\S 6.3)$. Here we consider also a precompact version of Protasov and Zelenyuk's construction [88] of topologies making a fixed sequence converge to 0.

Section 7 is dedicated to Pontryagin-van Kampen duality. In $\S \S 7.1-7.3$ we construct all tools for proving the duality theorem 7.36. More specifically, $\S \S 7.1$ and 7.2 contain various properties of the dual groups that allow for an easier computation of the dual in many cases. Using further the properties of the dual, we see in $\S 7.3$ that many specific groups $G$ satisfy the duality theorem, i.e., $G \cong \widehat{\widehat{G}}$. In $\S 7.4$ we stess the fact that the isomorphism $G \cong \widehat{\widehat{G}}$ is natural by studying in detail the natural transformation $\omega_{G}: G \rightarrow \widehat{\widehat{G}}$ connecting the group with its bidual. It is shown in several steps that $\omega_{G}$ is an isomorphism, considering larger and larger classes of locally compact abelian groups $G$ where the duality theorem holds (elementary locally compact abelian groups, compact abelian groups, discrete abelian groups, compactly generated locally compact abelian groups). The last step uses the fact that the duality functor is exact, this permits us to use all previous steps in the general case.

In the Appendix we dedicate some time to several topics that are not discussed in the main body of the notes: uniqueness of the duality, dualities for non-abelian or non-locally compact-groups, some connection to the topological properties of compact group and dynamical systems.

A large number of exercises is given in the text to ease the understanding of the basic properties of group topologies and the various aspects of the duality theorem.

These notes are born out of two courses in the framework of the PhD programs at the Department of Mathematics at Milan University and the Department of Geometry and Topology at the Complutense University of Madrid held in April/May 2007. Among the participants there were various groups, interested in different fields. To partially satisfy the interest of the audience I included various parts that can be eventually skipped, at least during the first reading. For example, the reader who is not interested in non-abelian groups can skip $\S \S 3.2 .4$, the entire $\S 3.7$ and take all groups abelian in $\S \S 3$ and 4 (conversely, the reader interested in non-abelian groups or rings may dedicate more time to $\S \S 3.2 .4,3.7$ and consider the non-abelian case also in the first half of $\S 4.2$, see the footnote at the beginning of $\S 4.2$ ). For the category theorists $\S \S 4.3,5.1-5.3,6.2-6.3$ may have less interest, compared to $\S \S 3.1-3.9,4.2,6.1,7.1-7.4$ and 8.1-8.2. Finally, those interested to get as fast as possible to the proof of the duality theorem can skip $\S \S 3.2 .3,3.2 .4$ and 3.6-3.9 (in particular, the route $\S \S 5-7$ is possible for the reader with sufficient knowledge of topological groups).

Several favorable circumstances helped in creating these notes. My sincere thanks go to my colleagues V. Zambelli, E. Martín-Peinador, S. Kazangian, M. J. Chasco, M. G. Bianchi, L. Außenhofer, X. Domíngues, M. Bruguera, S. Trevijano, and E. Pacifici who made this course possible. The younger participants of the course motivated me with their constant activity and challenging questions. I thank them for their interst and patience. I thank also my PhD student at Udine University Anna Giordano Bruno who prepared a very preliminary version of these notes in 2005 .

This notes are dedicated to the memory of Ivan Prodanov whose original contributions to Pontryagin-van Kampen duality can hardly by overestimated. The line adopted here follows his approach from [84] and [36]. 
Dikran Dikranjan 


\section{Contents}

1 Introduction $\quad$ i

2 Background on topological spaces and abstract groups 1

2.1 Background on abelian groups . . . . . . . . . . . . . . . . . . . . 1

2.2 Background on topological spaces . . . . . . . . . . . . . . . . . . . . . . . 3

3 General properties of topological groups 5

3.1 Definition of a topological group . . . . . . . . . . . . . . . . . . . 5

3.2 Examples of group topologies . . . . . . . . . . . . . . . . . . . . . 6

3.2 .1 Linear topologies . . . . . . . . . . . . . . . . . . . . . . . . 7

3.2 .2 Topologies generated by characters . . . . . . . . . . . . . . . . . . 7

3.2.3 Pseudonorms and pseudometrics in a group . . . . . . . . . . . . . . . . . 8

3.2 .4 Permutation groups . . . . . . . . . . . . . . . . . . . 8

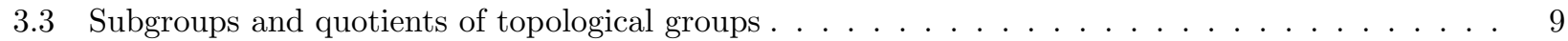

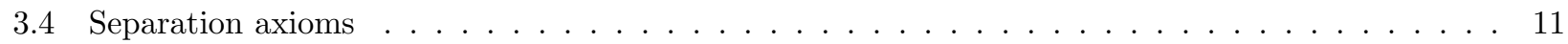

3.5 Connectedness in topological groups . . . . . . . . . . . . . . . . . . . . . . . . 12

3.6 Group topologies determined by sequences . . . . . . . . . . . . . . . . . . . . . . . 14

3.7 Markov's problems . . . . . . . . . . . . . . . . . . . . . . 15

3.7.1 The Zariski topology and the Markov topology . . . . . . . . . . . . . . . . . . 15

3.7.2 The Markov topology of the symmetric group . . . . . . . . . . . . . . . . . . 16

3.7.3 Existence of Hausdorff group topologies . . . . . . . . . . . . . . . . . . . . . . 18

3.7 .4 Extension of group topologies . . . . . . . . . . . . . . . . . . . . . . 19

3.8 Cardinal invariants of topological groups . . . . . . . . . . . . . . . . . . . . . . . 20

3.9 Completeness and completion . . . . . . . . . . . . . . . . . . . . . . . 22

4 Compactness and local compactness in topological groups 23

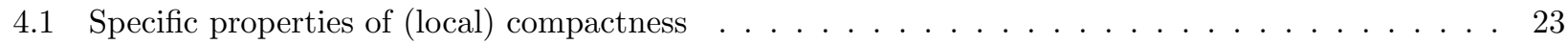

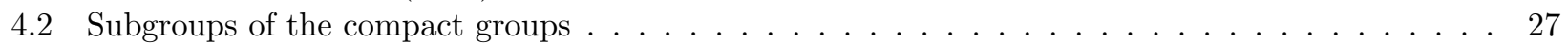

4.3 Subgroups of $\mathbb{R}^{n} \ldots \ldots \ldots \ldots \ldots \ldots$

5 Følner's theorem $\mathbf{3 2}$

5.1 Fourier theory for finite abelian groups . . . . . . . . . . . . . . . . . . . . 32

5.2 Bogoliouboff and Følner Lemmas . . . . . . . . . . . . . . . . . . . . . . . . 34

5.3 Prodanov's lemma and proof of Følner's theorem . . . . . . . . . . . . . . . . . . 37

6 Peter-Weyl's theorem and other applications of Følner's theorem 40

6.1 Precompact group topologies on abelian groups . . . . . . . . . . . . . . . . . . . . 40

6.2 Precompact group topologies determined by sequences . . . . . . . . . . . . . . . . . . . . 41

6.3 On the structure of compactly generated locally compact abelian groups . . . . . . . . . . . . . 43

7 Pontryagin-van Kampen duality 46

7.1 The dual group . . . . . . . . . . . . . . . . . . . . . . . . . . . 46

7.2 Computation of some dual groups . . . . . . . . . . . . . . . . . . . . . . . 48

7.3 Some general properties of the dual . . . . . . . . . . . . . . . . . . . . . . . 50

7.4 The natural transformation $\omega \ldots \ldots \ldots \ldots$

8 Appendix $\mathbf{5 8}$

8.1 Uniqueness of Pontryagin-van Kampen duality . . . . . . . . . . . . . . . . . . . . . . . . . . . . . . . . . . . .

8.2 Non-abelian or non-locally compact groups . . . . . . . . . . . . . . . . . . . . . 59

8.3 Relations to the topological theory of topological groups . . . . . . . . . . . . . . . . . . 59

8.4 Relations to dynamical systems . . . . . . . . . . . . . . . . . . . . . . . 59

$\begin{array}{ll}\text { Index } & 61 \\ -\end{array}$ 


\section{Notation and terminology}

We denote by $\mathbb{P}, \mathbb{N}$ and $\mathbb{N}_{+}$respectively the set of primes, the set of natural numbers and the set of positive integers. The symbol $\mathfrak{c}$ stands for the cardinality of the continuum. The symbols $\mathbb{Z}, \mathbb{Q}, \mathbb{R}, \mathbb{C}$ will denote the integers, the rationals, the reals and the complex numbers, respectively.

The quotient $\mathbb{T}=\mathbb{R} / \mathbb{Z}$ is a compact divisible abelian group, topologically isomorphic to the unitary circle $\mathbb{S}$ (i.e., the subgroup of all $z \in \mathbb{C}$ with $|z|=1$ ). For $\mathbb{S}$ we use the multiplicative notation, while for $\mathbb{T}$ we use the additive notation.

For an abelian group $G$ we denote by $\operatorname{Hom}(G, \mathbb{T})$ the group of all homomorphisms from $G$ to $\mathbb{T}$ written additively. The multiplicative form $G^{*}=\operatorname{Hom}(G, \mathbb{S}) \cong \operatorname{Hom}(G, \mathbb{T})$ will be used when necessary (e.g., concerning easier computation in $\mathbb{C}$, etc. $)$. We call the elements of $\operatorname{Hom}(G, \mathbb{T}) \cong \operatorname{Hom}(G, \mathbb{S})$ characters.

For a topological group $G$ we denote by $c(G)$ the connected component of the identity 1 in $G$. If $c(G)$ is trivial, the group $G$ is said to be totally disconnected. If $M$ is a subset of $G$ then $\langle M\rangle$ is the smallest subgroup of $G$ containing $M$ and $\bar{M}$ is the closure of $M$ in $G$. The symbol $w(G)$ stands for the weight of $G$. Moreover $\widetilde{G}$ stands for the completion of a Hausdorff topological abelian group $G$ (see $§ 3.9$ ).

\section{Background on topological spaces and abstract groups}

\subsection{Background on abelian groups}

Generally a group $G$ will be written multiplicatively and the neutral element will be denoted by $e_{G}$ or simply $e$ or 1 when there is no danger of confusion. For a subset $A, A_{1}, A_{2}, \ldots, A_{n}$ of a group $G$ we write

$$
A^{-1}=\left\{a^{-1}: a \in A\right\}, \quad \text { and } A_{1} A_{2} \ldots A_{n}=\left\{a_{1} \ldots a_{n}: a_{i} \in A_{i}, i=1,2, \ldots, n\right\}
$$

and we write $A^{n}$ for $A_{1} A_{2} \ldots A_{n}$ if all $A_{i}=A$. Moreover, for $A \subseteq G$ we denote by $c_{G}(A)$ the centralizer of $A$, i.e., the subgroup $\{x \in G: x a=a x$ for every $a \in A\}$.

We use additive notation for abelian groups, consequently 0 will denote the neutral element in such a case. Clearly, the counterpart of $(*)$ will be $-A, A_{1}+A_{2}+\ldots+A_{n}$ and $n A$.

A standard reference for abelian groups is the monograph [46]. We give here only those facts or definitions that appear very frequently in the sequel.

For $m \in \mathbb{N}_{+}$, we use $\mathbb{Z}_{m}$ or $\mathbb{Z}(m)$ for the finite cyclic group of order $m$. Let $G$ be an abelian group. The subgroup of torsion elements of $G$ is $t(G)$ and for $m \in \mathbb{N}_{+}$

$$
G[m]=\{x \in G: m x=0\} \quad \text { and } \quad m G=\{m x: x \in G\} .
$$

For a family $\left\{G_{i}: i \in I\right\}$ of groups we denote by $\prod_{i \in I} G_{i}$ the direct product $G$ of the groups $G_{i}$. The underlying set of $G$ is the Cartesian product $\prod_{i \in I} G_{i}$ and the operation is defined coordinatewise. The direct sum $\bigoplus_{i \in I} G_{i}$ is the subgroup of $\prod_{i \in I} G_{i}$ consisting of all elements of finite support. If all $G_{i}$ are isomorphic to the same group $G$ and $|I|=\alpha$, we write $\bigoplus_{\alpha} G$ (or $G^{(\alpha)}$, or $\bigoplus_{I} G$ ) for the direct sum $\bigoplus_{i \in I} G_{i}$

A subset $X$ of an abelian group $G$ is independent, if $\sum_{i=1}^{n} k_{i} x_{i}=0$ with $k_{i} \in \mathbb{Z}$ and distinct elements $x_{i}$ of $X, i=1,2, \ldots, n$, imply $k_{1}=k_{2}=\ldots=k_{n}=0$. The maximum size of an independent subset of $G$ is called free-rank of $G$ and denoted by $r_{0}(G)$. An abelian group $G$ is free, if $G$ has an independent set of generators $X$. In such a case $G \cong \bigoplus_{|X|} \mathbb{Z}$.

For an abelian group $G$ and a prime number $p$ the subgroup $G[p]$ is a vector space over the finite field $\mathbb{Z} / p \mathbb{Z}$. We denote by $r_{p}(G)$ its dimension over $\mathbb{Z} / p \mathbb{Z}$ and call it $p$-rank of $G$.

Let us start with the structure theorem for finitely generated abelian groups.

Theorem 2.1. If $G$ is a finitely generated abelian group, then $G$ is a finite direct product of cyclic groups. Moreover, if $G$ has $m$ generators, then every subgroup of $G$ is finitely generated as well and has at most $m$ generators.

Definition 2.2. An abelian group $G$ is

(a) torsion if $t(G)=G$;

(b) torsion-free if $t(G)=0$;

(c) bounded if $m G=0$ for some $m>0$;

(d) divisible if $G=m G$ for every $m>0$; 
(e) reduced if the only divisible subgroup of $G$ is the trivial one.

Example 2.3. (a) The groups $\mathbb{Z}, \mathbb{Q}, \mathbb{R}$, and $\mathbb{C}$ are torsion-free. The class of torsion-free groups is stable under taking direct products and subgroups.

(b) The groups $\mathbb{Z}_{m} \mathbb{Q} / \mathbb{Z}$ are torsion. The class of torsion groups is stable under taking direct sums, subgroups and quotients.

(c) Let $m_{1}, m_{2}, \ldots, m_{k}>1$ be naturals and let $\alpha_{1}, \alpha_{2}, \ldots, \alpha_{k}$ be cardinal numbers. Then the group $\bigoplus_{i=1}^{k} \mathbb{Z}_{m}^{\left(\alpha_{i}\right)}$ is bounded. According to a theorem of Prüfer every bounded abelian group has this form [46]. This generalizes the Frobenius-Stickelberger theorem about the structure of the finite abelian groups (see Theorem $2.1)$.

Example 2.4. (a) The groups $\mathbb{Q}, \mathbb{R}, \mathbb{C}$, and $\mathbb{T}$ are divisible.

(b) For $p \in \mathbb{P}$ we denote be $\mathbb{Z}\left(p^{\infty}\right)$ the Prüfer group, namely the $p$-primary component of the torsion group $\mathbb{Q} / \mathbb{Z}$ (so that $\mathbb{Z}\left(p^{\infty}\right)$ has generators $\left.c_{n}=1 / p^{n}+\mathbb{Z}, n \in \mathbb{N}\right)$. The group $\mathbb{Z}\left(p^{\infty}\right)$ is divisible.

(c) The class of divisible groups is stable under taking direct products, direct sums and quotients. In particular, every abelian group has a maximal divisible subgroup $d(G)$.

(d) [46] Every divisible group $G$ has the form $\left(\bigoplus_{r_{0}(G)} \mathbb{Q}\right) \oplus\left(\bigoplus_{p \in \mathbb{P}} \mathbb{Z}\left(p^{\infty}\right)^{\left(r_{p}(G)\right)}\right)$.

If $X$ is a set, a set $Y$ of functions of $X$ to a set $Z$ separates the points of $X$ if for every $x, y \in X$ with $x \neq y$, there exists $f \in Y$ such that $f(x) \neq f(y)$. Now we see that the characters separate the points of a discrete abelian groups.

Theorem 2.5. Let $G$ be an abelian group, $H$ a subgroup of $G$ and $D$ a divisible abelian group. Then for every homomorphism $f: H \rightarrow D$ there exists a homomorphism $\bar{f}: G \rightarrow D$ such that $\bar{f} \uparrow_{H}=f$.

If $a \in G \backslash H$ and $D$ contains elements of arbitrary finite order, then $\bar{f}$ can be chosen such that $\bar{f}(a) \neq 0$.

Proof. Let $H^{\prime}$ be a subgroup of $G$ such that $H^{\prime} \supseteq H$ and suppose that $g: H^{\prime} \rightarrow D$ is such that $g\lceil H=f$. We prove that for every $x \in G$, defining $N=H^{\prime}+\langle x\rangle$, there exists $\bar{g}: N \rightarrow D$ such that $\bar{g}\left\lceil_{H^{\prime}}=g\right.$. There are two cases.

If $\langle x\rangle \cap H^{\prime}=\{0\}$, then take any $y \in D$ and define $\bar{g}(h+k x)=g(h)+k y$ for every $h \in H^{\prime}$ and $k \in \mathbb{Z}$. Then $\bar{g}$ is a homomorphism. This definition is correct because every element of $N$ can be represented in a unique way as $h+k x$, where $h \in H^{\prime}$ and $k \in \mathbb{Z}$.

If $C=\langle x\rangle \cap H^{\prime} \neq\{0\}$, then $C$ is cyclic, being a subgroup of a cyclic group. So $C=\langle l x\rangle$ for some $l \in \mathbb{Z}$. In particular, $l x \in H^{\prime}$ and we can consider the element $a=g(l x) \in D$. Since $D$ is divisible, there exists $y \in D$ such that $l y=a$. Now define $\bar{g}: N \rightarrow D$ putting $\bar{g}(h+k y)=g(h)+k y$ for every $h+k x \in N$, where $h \in H^{\prime}$ and $k \in \mathbb{Z}$. To see that this definition is correct, suppose that $h+k x=h^{\prime}+k^{\prime} x$ for $h, h^{\prime} \in H^{\prime}$ and $k, k^{\prime} \in \mathbb{Z}$. Then $h-h^{\prime}=k^{\prime} x-k x=\left(k^{\prime}-k\right) x \in C$. So $k-k^{\prime}=s l$ for some $s \in \mathbb{Z}$. Since $g: H^{\prime} \rightarrow D$ is a homomorphism and $l x \in H^{\prime}$, we have

$$
g(h)-g\left(h^{\prime}\right)=g\left(h-h^{\prime}\right)=g(s(l x))=s g(l x)=s a=s l y=\left(k^{\prime}-k\right) y=k^{\prime} y-k y .
$$

Thus, from $g(h)-g\left(h^{\prime}\right)=k^{\prime} y-k y$ we conclude that $g(h)+k y=g\left(h^{\prime}\right)+k^{\prime} y$. Therefore $\bar{g}$ is correctly defined. Moreover $\bar{g}$ is a homomorphism and extends $g$.

Let $\mathcal{M}$ be the family of all subgroups $H_{i}$ of $G$ such that $H \leq H_{i}$ and of all homomorphisms $f_{i}: H_{i} \rightarrow D$ that extend $f: H \rightarrow D$. For $\left(H_{i}, f_{i}\right),\left(H_{j}, f_{j}\right) \in \mathcal{M}$ put $\left(H_{i}, f_{i}\right) \leq\left(H_{j}, f_{j}\right)$ if $H_{i} \leq H_{j}$ and $f_{j}$ extends $f_{i}$. In this way $(\mathcal{M}, \leq)$ is partially ordered. Let $\left\{\left(H_{i}, f_{i}\right)\right\}_{i \in I}$ a totally ordered subset of $(\overline{\mathcal{M}}, \leq)$. Then $H_{0}=\bigcup_{i \in I} H_{i}$ is a subgroup of $G$ and $f_{0}: H_{0} \rightarrow D$ defined by $f_{0}(x)=f_{i}(x)$ whenever $x \in H_{i}$, is a homomorphism that extends $f_{i}$ for every $i \in I$. This proves that $(\mathcal{M}, \leq)$ is inductive and so we can apply Zorn's lemma to find a maximal element $\left(H_{\max }, f_{\max }\right)$ of $(\mathcal{M}, \leq)$. It is easy to see that $H_{\max }=G$.

Suppose now that $D$ contains elements of arbitrary finite order. If $a \in G \backslash H$, we can extend $f$ to $H+\langle a\rangle$ defining it as in the first part of the proof. If $\langle a\rangle \cap H=\{0\}$ then $\bar{f}(h+k a)=f(h)+k y$ for every $k \in \mathbb{Z}$, where $y \in D \backslash\{0\}$. If $\langle a\rangle \cap H \neq\{0\}$, since $D$ contains elements of arbitrary order, we can choose $y \in D$ such that $\bar{f}(h+k a)=f(h)+k y$ with $y \neq 0$. In both cases $\bar{f}(a)=y \neq 0$.

Corollary 2.6. Let $G$ be an abelian group and $H$ a subgroup of $G$. If $\chi \in \operatorname{Hom}(H, \mathbb{T})$ and $a \in G \backslash H$, then $\chi$ can be extended to $\bar{\chi} \in \operatorname{Hom}(G, \mathbb{T})$, with $\bar{\chi}(a) \neq 0$. 
Corollary 2.7. If $G$ is an abelian group, then $\operatorname{Hom}(G, \mathbb{T})$ separates the points of $G$.

Corollary 2.8. If $G$ is an abelian group and $D$ a divisible subgroup of $G$, then there exists a subgroup $B$ of $G$ such that $G=D \times B$.

Proof. Consider the homomorphism $f: D \rightarrow G$ defined by $f(x)=x$ for every $x \in D$. By Theorem 2.5 we can extend $f$ to $\bar{f}: G \rightarrow G$. Then put $B=\operatorname{ker} \bar{f}$ and observe that $G=D+B$ and $D \cap B=\{0\}$; consequently $G \cong D \times B$.

Corollary 2.9. Every abelian group $G$ can be written as $G=d(G) \times R$, where $R T$ is a reduced subgroup of $G$.

Proof. By Corollary 2.8 there exists a subgroup $R$ of $G$ such that $G=d(G) \times R$. To conclude that $R$ is reduced it suffices to apply the definition of $d(G)$.

The ring of endomorphisms of the group $\mathbb{Z}\left(p^{\infty}\right)$ will be denoted by $\mathbb{J}_{p}$, it is isomorphic the inverse limit $\lim _{\leftarrow} \mathbb{Z} / p^{n} \mathbb{Z}$, known also as the ring of $p$-adic integers. The field of quotients of $\mathbb{J}_{p}$ (i.e., the field of $p$-adic numbers) will be denoted by $\mathbb{Q}_{p}$. Sometimes we shall consider only the underlying groups of these rings (and speak of "the group $p$-adic integers", or "the group $p$-adic numbers).

\subsection{Background on topological spaces}

We assume the reader is familiar with the basic definitions and notions related to topological spaces. For the sake of completeness we recall here some frequently used properties related to compactness.

Definition 2.10. A topological space $X$ is

- compact if for every open cover of $X$ there exists a finite subcover;

- Lindelöff if for every open cover of $X$ there exists a countable subcover;

- locally compact if every point of $X$ has compact neighborhood in $X$;

- $\sigma$-compact if $X$ is the union of countably many compact subsets;

- of first category, if $X=\bigcup_{n=1}^{\infty} A_{n}$ and every $A_{n}$ is a closed subset of $X$ with empty interior;

- of second category, if $X$ is not of first category;

- connected if for every proper open subset of $X$ with open complement is empty.

Here we recall properties of maps:

Definition 2.11. For a map $f:(X, \tau) \rightarrow\left(Y, \tau^{\prime}\right)$ between topological spaces and a point $x \in X$ we say:

- $f$ is continuous at $x$ if for every neighborhood $U$ of $f(x)$ in $Y$ there exists a neighborhood $V$ of $x$ in $X$ such that $f(V) \subseteq U$,

- $f$ is open in $x \in X$ if for every neighborhood $V$ of $x$ in $X$ there exists a neighborhood $U$ of $f(x)$ in $Y$ such that $f(V) \supseteq U$,

- $f$ is continuous (resp., open) if $f$ is continuous (resp., open) at every point $x \in X$.

- $f$ is closed if the subset $f(A)$ of $Y$ is closed for every closed subset $A \subseteq X$.

Some basic properties relating spaces to continuous maps are collected in the next lemma:

Lemma 2.12. - If $f: X \rightarrow Y$ is a continuous surjective map, then $Y$ is compact (resp., Lindelöff, $\sigma$ compact, connected) whenever $X$ has the same property.

- If $X$ is a closed subspace of a space $Y$, then $X$ is compact (resp., Lindelöff, $\sigma$-compact, locally compact) whenever $Y$ has the same property.

- If $X=\prod_{i \in I} X_{i}$, then $X$ is compact (resp., connected) iff every space $X_{i}$ has the same property. If I is finite, the same holds for local compactness and $\sigma$-compactness. 
A partially ordered set $(A, \leq)$ is directed if for every $\alpha, \beta \in A$ there exists $\gamma \in A$ such that $\gamma \geq \alpha$ and $\gamma \geq \beta$. A subset $B$ of $A$ is cofinal, if for every $\alpha \in A$ there exists $\beta \in B$ with $\beta \geq \alpha$.

A net in a topological space $X$ is a map from a directed set $A$ to $X$. We write $x_{\alpha}$ for the image of $\alpha \in A$ so that the net can be written in the form $N=\left\{x_{\alpha}\right\}_{\alpha \in A}$. A subnet of a net $N$ is $S=\left\{x_{\beta}\right\}_{\beta \in B}$ such that $B$ is a cofinal subset of $A$.

A net $\left\{x_{\alpha}\right\}_{\alpha \in A}$ in $X$ converges to $x \in X$ if for every neighborhood $U$ of $x$ in $X$ there exists $\beta \in A$ such that $\alpha \in A$ and $\alpha \geq \beta$ implies $\alpha \in U$.

Lemma 2.13. Let $X$ be a topological space.

(a) If $Z$ is a subset of $X$, then $x \in \bar{Z}$ if and only if there exists a net in $Z$ converging to $x$.

(b) $X$ is compact if and only if every net in $X$ has a convergent subnet.

(c) A function $f: X \rightarrow Y$ (where $Y$ is a topological space) is continuous if and only if $f\left(x_{\alpha}\right) \rightarrow f(x)$ in $Y$ for every net $\left\{x_{\alpha}\right\}_{\alpha \in A}$ in $X$ with $x_{\alpha} \rightarrow x$.

(d) The space $X$ is Hausdorff if and only if every net in $X$ converges to at most one point in $X$.

Let us recall that the connected component of a point $x$ in a topological space $X$ is the largest connected subset of $X$ containing $x$. It is always a closed subset of $X$. The space $X$ is called totally disconnected if all connected components are singletons.

In a topological space $X$ the quasi-component of a point $x \in X$ is the intersection of all clopen sets of $X$ containing $x$.

Lemma 2.14. (Shura-Bura) In a compact space $X$ the quasi-components and the connected components coincide.

A topological space $X$ zero-dimensional if $X$ has a base of clopen sets. Zero-dimensional $T_{2}$ spaces are totally disconnected (as every point is an intersection of clopen sets).

Theorem 2.15. (Vedenissov) Every totally disconnected locally compact space is zero-dimensional.

By $\beta X$ we denote the $\check{C}$ ech-Stone compactification of a topological Tychonov space $X$, that is the compact space $\beta X$ together with the dense immersion $i: X \rightarrow \beta X$, such that for every function $f: X \rightarrow[0,1]$ there exists $f^{\beta}: \beta X \rightarrow[0,1]$ which extends $f$ (this is equivalent to ask that every function of $X$ to a compact space $Y$ can be extended to $\beta X)$. Here $\beta X$ will be used only for a discrete space $X$.

Theorem 2.16 (Baire category theorem). A Hausdorff locally compact space $X$ is of second category.

Proof. Suppose that $X=\bigcup_{n=1}^{\infty} A_{n}$ and assume that every $A_{n}$ is closed with empty interior. Then the sets $D_{n}=G \backslash A_{n}$ are open and dense in $X$. To get a contradiction, we show that $\bigcap_{n=1}^{\infty} D_{n}$ is dense, in particular non-empty (so $G \neq \bigcup_{n=1}^{\infty} A_{n}$, a contradiction).

We use the fact that a Hausdorff locally compact space is regular. Pick an arbitrary open set $V \neq \emptyset$. Then there exists an open set $U_{0} \neq \emptyset$ with $\bar{U}_{0}$ compact and $\bar{U}_{0} \subseteq V$. Since $D_{1}$ is dense, $U_{0} \cap D_{1} \neq \emptyset$. Pick $x_{1} \in U_{0} \cap D_{1}$ and an open set $U_{1} \ni x_{1}$ in $X$ with $\bar{U}_{1}$ compact and $\bar{U}_{1} \subseteq U_{0} \cap D_{1}$. Proceeding in this way, for every $n \in \mathbb{N}_{+}$ we can find an open set $U_{n} \neq \emptyset$ in $G$ with $\bar{U}_{n}$ compact and $\bar{U}_{n} \subseteq U_{n-1} \cap D_{n}$. By the compactness of every $\bar{U}_{n}$ there exists a point $x \in \bigcap_{n=1}^{\infty} \bar{U}_{n}$. Obviously, $x \in V \cap \bigcap_{n=1}^{\infty} D_{n}$.

Lemma 2.17. If $G$ is a locally compact $\sigma$-compact space, then $G$ is a Lindelöff space.

Proof. Let $G=\bigcup_{\alpha \in I} U_{\alpha}$. Since $G$ is $\sigma$-compact, $G=\bigcup_{n=1}^{\infty} K_{n}$ where each $K_{n}$ is a compact subset of $G$. Thus for every $n \in \mathbb{N}_{+}$there exists a finite subset $F_{n}$ of $I$ such that $K_{n} \subseteq \bigcup_{n \in F_{n}} U_{n}$. Now $I_{0}=\bigcup_{n=1}^{\infty} F_{n}$ is a countable subset of $I$ and $K_{n} \subseteq \bigcup_{\alpha \in I_{0}} U_{\alpha}$ for every $N \in \mathbb{N}_{+}$yields $G=\bigcup_{\alpha \in I_{0}} U_{\alpha}^{n}$.

Let $X$ be a topological space. Let $\mathcal{C}(X, \mathbb{C})$ be the $\mathbb{C}$-algebra of all continuous complex valued functions on $X$. If $f \in \mathcal{C}(X, \mathbb{C})$ let

$$
\|f\|_{\infty}=\sup \{|f(x)|: x \in X\} .
$$

Theorem 2.18 (Stone-Weierstraß theorem). Let $X$ be a compact topological space. A $\mathbb{C}$-subalgebra $\mathcal{A}$ of $\mathcal{C}(X, \mathbb{C})$ containing all constants and closed under conjugation is dense in $\mathcal{C}(X, \mathbb{C})$ for the norm \|\|$_{\infty}$ if and only if $\mathcal{A}$ separates the points of $X$.

We shall need in the sequel the following local form of Stone-Weierstraß theorem. 
Corollary 2.19. Let $X$ be a compact topological space and $f \in \mathcal{C}(X, \mathbb{C})$. Then $f$ can be uniformly approximated by a $\mathbb{C}$-subalgebra $\mathcal{A}$ of $\mathcal{C}(X, \mathbb{C})$ containing all constants and closed under the complex conjugation if and only if $\mathcal{A}$ separates the points of $X$ separated by $f \in \mathcal{C}(X, \mathbb{C})$.

Proof. Denote by $G: X \rightarrow \mathbb{C}^{\mathcal{A}}$ the diagonal map of the family $\{g: g \in \mathcal{A}\}$. Then $Y=G(X)$ is a compact subspace of $\mathbb{C}^{\mathcal{A}}$ and by the compactness of $X$, its subspace topology coincides with the quotient topology of the map $G: X \rightarrow Y$. The equivalence relation $\sim$ in $X$ determined by this quotient is as follows: $x \sim y$ for $x, y \in X$ by if and only if $G(x)=G(y)$ (if and only if $g(x)=g(y)$ for every $g \in \mathcal{A}$ ). Clearly, every continuous function $h: X \rightarrow \mathbb{C}$, such that $h(x)=h(y)$ for every pair $x, y$ with $x \sim y$, can be factorized as $h=\bar{h} \circ q$, where $\bar{h} \in \mathcal{C}(Y, \mathbb{C})$. In particular, this holds true for all $g \in \mathcal{A}$ and for $f$ (for the latter case this follows from our hypothesis). The $\mathbb{C}$-subalgebra $\overline{\mathcal{A}} \subseteq \mathcal{C}(Y, \mathbb{C})$ is closed under the complex conjugation and contains all constants. It is easy to see that it separates the points of $Y$. Hence we can apply Stone - Weierstraß theorem 2.18 to $Y$ and $\overline{\mathcal{A}}$ to deduce that we can uniformly approximate the function $\bar{f}$ by functions of $\overline{\mathcal{A}}$. This produces uniform approximation of the function $f$ by functions of $\mathcal{A}$.

\section{General properties of topological groups}

\subsection{Definition of a topological group}

Let us start with the following fundamental concept:

Definition 3.1. Let $G$ be a group.

- A topology $\tau$ on $G$ is said to be a group topology if the map $f: G \times G \rightarrow G$ defined by $f(x, y)=x y^{-1}$ is continuous.

- A topological group is a pair $(G, \tau)$ of a group $G$ and a group topology $\tau$ on $G$.

If $\tau$ is Hausdorff (resp., compact, locally compact, connected, etc.), then the topological group $(G, \tau)$ is called Hausdorff (resp., compact, locally compact, connected, etc.). Analogously, if $G$ is cyclic (resp., abelian, nilpotent, etc.) the topological group $(G, \tau)$ is called cyclic (resp. abelian, nilpotent, etc.). Obviously, a topology $\tau$ on a group $G$ is a group topology iff the maps

$$
\mu: G \times G \rightarrow G \text { and } \iota: G \rightarrow G
$$

defined by $\mu(x, y)=x y$ and $\iota(x)=x^{-1}$ are continuous when $G \times G$ carries the product topology.

Here are some examples, starting with two trivial ones: for every group $G$ the discrete topology and the indiscrete topology on $G$ are group topologies. Non-trivial examples of a topological group are provided by the additive group $\mathbb{R}$ of the reals and by the multiplicative group $\mathbb{S}$ of the complex numbers $z$ with $|z|=1$, equipped both with their usual topology. This extends to all powers $\mathbb{R}^{n}$ and $\mathbb{S}^{n}$. These are abelian topological groups. For every $n$ the linear group $G L_{n}(\mathbb{R})$ equipped with the topology induced by $\mathbb{R}^{n^{2}}$ is a non-abelian topological group. The groups $\mathbb{R}^{n}$ and $G L_{n}(\mathbb{R})$ are locally compact, while $\mathbb{S}$ is compact.

Example 3.2. For every prime $p$ the group $\mathbb{J}_{p}$ of $p$-adic integers carries the topology induced by $\prod_{n=1}^{\infty} \mathbb{Z}\left(p^{n}\right)$, when we consider it as the inverse limit $\lim _{\leftarrow} \mathbb{Z} / p^{n} \mathbb{Z}$. The same topology can be obtained also when we consider $\mathbb{J}_{p}$ as the ring of all endomorphims of the group $\mathbb{Z}\left(p^{\infty}\right)$. Now $\mathbb{J}_{p}$ embeds into the product $\mathbb{Z}\left(p^{\infty}\right)^{\mathbb{Z}\left(p^{\infty}\right)}$ carrying the product topology, while $\mathbb{Z}\left(p^{\infty}\right)$ is discrete. We leave to the reader the verification that this is a compact group topology on $\mathbb{J}_{p}$. Basic open neighborhoods of 0 in this topology are the subgroups $p^{n} \mathbb{J}_{p}$ of $\left(\mathbb{J}_{p},+\right)$ (actually, these are ideals of the ring $\mathbb{J}_{p}$ ) for $n \in \mathbb{N}$. The field $\mathbb{Q}_{p}$ becomes a locally compact group by declaring $\mathbb{J}_{p}$ open in $\mathbb{Q}_{p}$ (i.e., an element $x \in \mathbb{Q}_{p}$ has as typical neighborhoods the cosets $x+p^{n} \mathbb{J}_{p}, n \in \mathbb{N}$.

Other examples of group topologies will be given in $\S 3.2$.

If $G$ is a topological group written multiplicatively and $a \in G$, then the translations $x \mapsto a x$ and $x \mapsto x a$ as well as the internal automorphism $x \mapsto a x a^{-1}$ are homeomorphisms. Consequently, the group $G$ is discrete iff the point 1 is isolated, i.e., the singleton $\{1\}$ is open. In the sequel $a M$ will denote the image of a subset $M \subseteq G$ under the (left) translation $x \mapsto a x$, i.e., $a M:=\{a m: m \in M\}$. This notation will be extended also to families of subsets of $G$, in particular, for every filter $\mathcal{F}$ we denote by $a \mathcal{F}$ the filter $\{a F: F \in \mathcal{F}\}$.

Making use of the homeomorphisms $x \mapsto a x$ one can prove:

Exercise 3.3. Let $f: G \rightarrow H$ be a homomorphism between topological groups. Prove that $f$ is continuous (resp., open) iff $f$ is continuous (resp., open) at $1 \in G$. 
For a topological group $G$ and $g \in G$ we denote by $\mathcal{V}_{G, \tau}(g)$ the filter of all neighborhoods of the element $g$ of $G$. When no confusion is possible, we shall write briefly also $\mathcal{V}_{G}(g), \mathcal{V}_{\tau}(g)$ or even $\mathcal{V}(g)$. Among these filters the filter $\mathcal{V}_{G, \tau}(1)$, obtained for the neutral element $g=1$, plays a central role. It is useful to note that for every $a \in G$ the filter $\mathcal{V}_{G}(a)$ coincides with $a \mathcal{V}_{G}(1)=\mathcal{V}_{G}(1) a$. More precisely, we have the following:

Theorem 3.4. Let $G$ be a group and let $\mathcal{V}(1)$ be the filter of all neighborhoods of 1 in some group topology $\tau$ on $G$. Then:

(a) for every $U \in \mathcal{V}(1)$ there exists $V \in \mathcal{V}(1)$ with $V \cdot V \subseteq U$;

(b) for every $U \in \mathcal{V}(1)$ there exists $V \in \mathcal{V}(1)$ with $V^{-1} \subseteq U$;

(c) for every $U \in \mathcal{V}(1)$ and for every $a \in G$ there exists $V \in \mathcal{V}(1)$ with $a V a^{-1} \subseteq U$.

Conversely, if $\mathcal{V}$ is a filter on $G$ satisfying (a), (b) and (c), then there exists a unique group topology $\tau$ on $G$ such that $\mathcal{V}$ coincides with the filter of all $\tau$-neighborhoods of 1 in $G$.

Proof. To prove (a) it suffices to apply the definition of the continuity of the multiplication $\mu: G \times G \rightarrow G$ at $(1,1) \in G \times G$. Analogously, for (b) use the continuity of the map $\iota: G \rightarrow G$ at $1 \in G$. For item (c) use the continuity of the internal automorphism $x \mapsto a x a^{-1}$ at $1 \in G$.

Let $\mathcal{V}$ be a filter on $G$ satisfying all conditions (a), (b) and (c). Let us see first that every $U \in \mathcal{V}$ contains 1 . In fact, take $W \in \mathcal{V}$ with $W \cdot W \subseteq U$ and choose $V \in \mathcal{V}(1)$ with $V \subseteq W$ and $V^{-1} \subseteq W$. Then $1 \in V \cdot V^{-1} \subseteq U$.

Now define a topology $\tau$ on $G$ whose open sets $O$ are defined by the following property:

$$
\tau:=\{O \subseteq G:(\forall a \in O)(\exists U \in \mathcal{V}) \text { such that } a U \subseteq O\}
$$

It is easy to see that $\tau$ is a topology on $G$. Let us see now that for every $g \in G$ the filter $g \mathcal{V}$ coincides with the filter $\mathcal{V}_{(G, \tau)}(g)$ of all $\tau$-neighborhoods of $g$ in $(G, \tau)$. The inclusion $g \mathcal{V} \supseteq \mathcal{V}_{(G, \tau)}(g)$ is obvious. Assume $U \in \mathcal{V}$. To see that $g U \in \mathcal{V}_{(G, \tau)}(g)$ we have to find a $\tau$-open $O \subseteq g U$ that contains $g$. Let $O:=\{h \in g U:(\exists W \in \mathcal{V}) h W \subseteq g U\}$. Obviously $g \in O$. To see that $O \in \tau$ pick $x \in O$. Then there exists $W \in \mathcal{V}$ with $x W \subseteq g U$. Let $V \in \mathcal{V}$ with $V \cdot V \subseteq W$, then $x V \subseteq O$ since $x v V \subseteq g U$ for every $v \in V$.

We have seen that $\tau$ is a topology on $G$ such that the $\tau$-neighborhoods of any $x \in G$ are given by the filter $x \mathcal{V}$. It remains to see that $\tau$ is a group topology. To this end we have to prove that the map $(x, y) \mapsto x y^{-1}$ is continuous. Fix $x, y$ and pick a $U \in \mathcal{V}$. By (c) there exists a $W \in \mathcal{V}$ with $W y^{-1} \subseteq y^{-1} U$. Now choose $V \in \mathcal{V}$ with $V \cdot V^{-1} \subseteq W$. Then $O=x V \times y V$ is a neighborhood of $(x, y)$ in $G \times G$ and $f(O) \subseteq x V \cdot V^{-1} y^{-1} \subseteq$ $x W y^{-1} \subseteq x y^{-1} U$

In the above theorem one can take instead of a filter $\mathcal{V}$ also a filter base, i.e., a family $\mathcal{V}$ with the property

$$
(\forall U \in \mathcal{V})(\forall V \in \mathcal{V})(\exists W \in \mathcal{V}) W \subseteq U \cap V
$$

beyond the proprieties (a)-(c).

A neighborhood $U \in \mathcal{V}(1)$ is symmetric, if $U=U^{-1}$. Obviously, for every $U \in \mathcal{V}(1)$ the intersection $U \cap U^{-1} \in \mathcal{V}(1)$ is a symmetric neighborhood, hence every neighborhood of 1 contains a symmetric one.

Let $\left\{\tau_{i}: i \in I\right\}$ be a family of group topologies on a group $G$. Then their supremum $\tau=\sup _{i \in I} \tau_{i}$ is a group topology on $G$ with a base of neighborhoods of 1 formed by the family of all finite intersection $U_{1} \cap U_{2} \cap \ldots \cap U_{n}$, where $U_{k} \in \mathcal{V}_{\tau_{i_{k}}}(1)$ for $k=1,2, \ldots, n$ and the $n$-tuple $i_{1}, i_{2}, \ldots, i_{n}$ runs over all finite subsets if $I$.

Exercise 3.5. If $\left(a_{n}\right)$ is a sequence in $G$ such that $a_{n} \rightarrow 1$ for every member $\tau_{i}$ of a family $\left\{\tau_{i}: i \in I\right\}$ of group topologies on a group $G$, then $a_{n} \rightarrow 1$ also for the supremum $\sup _{i \in I} \tau_{i}$.

\subsection{Examples of group topologies}

Now we give several series of examples of group topologies, introducing them by means of the filter $\mathcal{V}(1)$ of neighborhoods of 1 as explained above. However, in all cases we avoid the treat the whole filter $\mathcal{V}(1)$ and we prefer to deal with an essential part of it, namely a base. Let us recall the precise definition of a base of neighborhoods.

Definition 3.6. Let $G$ be a topological group. A family $\mathcal{B} \subseteq \mathcal{V}(1)$ is said to be a base of neighborhoods of 1 (or briefly, a base at 1) if for every $U \in \mathcal{V}(1)$ there exists a $V \in \mathcal{B}$ contained in $U$ (such a family will necessarily be a filterbase). 


\subsubsection{Linear topologies}

Let $\mathcal{V}=\left\{N_{i}: i \in I\right\}$ be a filter base consisting of normal subgroups of a group $G$. Then $\mathcal{V}$ satisfies (a)-(c), hence generates a group topology on $G$ having as basic neighborhoods of a point $g \in G$ the family of cosets $\left\{g N_{i}: i \in I\right\}$. Group topologies of this type will be called linear topologies. Let us see now various examples of linear topologies.

Example 3.7. Let $G$ be a group and let $p$ be a prime:

- the pro-finite topology, with $\left\{N_{i}: i \in I\right\}$ all normal subgroups of finite index of $G$;

- the pro-p-finite topology, with $\left\{N_{i}: i \in I\right\}$ all normal subgroups of $G$ of finite index that is a power of $p$;

- the $p$-adic topology, with $I=\mathbb{N}$ and for $n \in \mathbb{N}, N_{n}$ is the subgroup (necessarily normal) of $G$ generated by all powers $\left\{g^{p^{n}}: g \in G\right\}$.

- the natural topology (or $\mathbb{Z}$-topology), with $I=\mathbb{N}$ and for $n \in \mathbb{N}, N_{n}$ is the subgroup (necessarily normal) of $G$ generated by all powers $\left\{g^{n}: g \in G\right\}$.

- the pro-countable topology, with $\left\{N_{i}: i \in I\right\}$ all normal subgroups of at most countable index $\left[G: N_{i}\right]$.

The next simple construction belongs to Taimanov. Now neighborhoods of 1 are subgroups, that are not necessarily normal.

Exercise 3.8. Let $G$ be a group with trivial center. Then $G$ can be considered as a subgroup of Aut $(G)$ making use of the internal automorphisms. Identify Aut $(G)$ with a subgroup of the power $G^{G}$ and equip Aut $(G)$ with the group topology $\tau$ induced by the product topology of $G^{G}$, where $G$ carries the discrete topology. Prove that:

- the filter of all $\tau$-neighborhoods of 1 has as base the family of centralizers $\left\{c_{G}(F)\right\}$, where $F$ runs over all finite subsets of $G$;

- $\tau$ is Hausdorff;

- $\tau$ is discrete iff there exists a finite subset of $G$ with trivial centralizer.

\subsubsection{Topologies generated by characters}

Let $G$ be an abelian group. A character of $G$ is a homomorphism $\chi: G \rightarrow \mathbb{S}$. For characters $\chi_{i}, i=1, \ldots, n$, of $G$ and $\delta>0$ let

$$
U_{G}\left(\chi_{1}, \ldots, \chi_{n} ; \delta\right):=\left\{x \in G:\left|\operatorname{Arg}\left(\chi_{i}(x)\right)\right|<\delta, i=1, \ldots, n\right\},
$$

where the argument $\operatorname{Arg}(z)$ of a complex number $z$ is taken in $(-\pi, \pi]$.

Exercise 3.9. Let $G$ be an abelian group and let $H$ be a family of characters of $G$. Then the family

$$
\left\{U_{G}\left(\chi_{1}, \ldots, \chi_{n} ; \delta\right): \delta>0, \chi_{i} \in H, i=1, \ldots, n\right\}
$$

is a filter base satisfying the conditions (a)-(c) of Theorem 3.4, hence it gives rise to a group topology $\mathcal{T}_{H}$ on $G$ (this is the initial topology of the family $H$, i.e., the coarsest topology that makes continuous all the characters of $H$ ).

We refer to the group topology $\mathcal{T}_{H}$ as topology generated by the characters of $H$. The topology $\mathcal{T}_{G^{*}}$, generated by all characters of $G$, is called Bohr topology of $G$.

For an abelian group $G$ some of the linear topologies on $G$ are also generated by appropriate families of characters.

Exercise 3.10. Let $G$ be an abelian group.

1. Prove that the profinite topology of $G$ is contained in the Bohr topology of $G$. Give an example of a group $G$ where these two topologies differ.

2. Let $H$ be the family of all characters $\chi$ of $G$ such that the subgroup $\chi(G)$ is finite. Prove that the topology $\mathcal{T}_{H}$ coincides with the pro-finite topology on $G$.

3. Let $H$ be the family of all characters $\chi$ of $G$ such that the subgroup $\chi(G)$ is finite and contained in the subgroup $\mathbb{Z}\left(p^{\infty}\right)$ of $\mathbb{T}$. Prove that the topology $\mathcal{T}_{H}$ coincides with the pro-p-finite topology on $G$. 
This exercise suggests to call a character $\chi: G \rightarrow \mathbb{T}$ torsion is there exists $n>0$ such that $\chi$ vanishes on the subgroup $n G:=\{n x: x \in G\}$. (Equivalently, the character $n \cdot \chi$ coincides with the trivial character, where the character $n \cdot \chi: G \rightarrow \mathbb{T}$ is defined by $(n \cdot \chi)(x):=n \chi(x)$.)

Exercise 3.11. Let $G$ be an abelian group. Prove that:

1. if $H$ is a family of characters of $G$, then the topology $\mathcal{T}_{H}$ is contained in the pro-finite topology of $G$ iff every character of $H$ is torsion.

2. if $G$ is bounded, then the Bohr topology of $G$ coincides with the profinite topology of $G$.

3. if the Bohr topology of $G$ coincides with the profinite topology of $G$, then $G$ is bounded.

\subsubsection{Pseudonorms and pseudometrics in a group}

According to Markov a pseudonorm in an abelian group $G$ is a map $\nu: G \rightarrow \mathbb{R}_{+}$such that for every $x, y \in G$ :

(1) $\nu(1)=0$;

(2) $\nu\left(x^{-1}\right)=\nu(x)$;

(3) $\nu(x y) \leq \nu(x)+\nu(y)$.

The norms defined in a real vector space are obviously pseudonorms (with the additional property, in additive notation, $\nu(0)=0$ iff $x=0)$.

Every pseudonorm $\nu$ generates a pseudometric $d_{\nu}$ on $G$ defined by $d_{\nu}(x, y):=\nu\left(x^{-1} y\right)$. This pseudometric is left invariant in the sense that $d_{\nu}(a x, a y)=d_{\nu}(x, y)$ for every $a, x, y \in G$. Denote by $\tau_{\nu}$ the topology induced on $G$ by this pseudometric. A base of $\mathcal{V}_{\tau_{\nu}}(1)$ is given by the open balls $\left\{B_{1 / n}(1): n \in \mathbb{N}_{+}\right\}$.

In order to build metrics inducing the topology of a given topological group $(G, \tau)$ we need the following lemma (for a proof see $[67,8.2],[79]$ ). We say that a pseudometric $d$ on $G$ is continuous if the map $d: G \times G \rightarrow \mathbb{R}_{+}$ is continuous. This is equivalent to have the topology induced by the metric $d$ coarser than the topology $\tau$ (i.e., every open set with respect to the metric $d$ is $\tau$-open).

Lemma 3.12. Let $G$ be a topological group and let

$$
U_{0} \supseteq U_{1} \supseteq \ldots \supseteq U_{n} \supseteq \ldots
$$

be symmetric neighborhoods of 1 with $U_{n}^{3} \subseteq U_{n-1}$ for every $n \in \mathbb{N}$. Then there exists a continuous left invariant pseudometric d on $G$ such that $U_{n} \subseteq B_{1 / n}(1) \subseteq U_{n-1}$ for every $n$.

Exercise 3.13. Prove that in the previous lemma $H=\bigcap_{n=1}^{\infty} U_{n}$ is a closed subgroup of $G$ with the property $H=\{x \in G: d(x, 1)=0\}$. In particular, $d$ is a metric iff $H=\{1\}$.

If the chain (2) has also the property $x U_{n} x^{-1} \subseteq U_{n-1}$ for every $x \in G$ and for every $n$, the subgroup $H$ is normal and $d$ defines a metric on the quotient group letting $\tilde{d}(x H, y H):=d(x, y)$. The metric $\tilde{d}$ induces the quotient topology on $G / H$.

\subsubsection{Permutation groups}

Let $X$ be an infinite set and let $G$ briefly denotes the group $S(X)$ of all permutations of $X$. A very natural topology on $G$ is defined by taking as filter of neighborhoods of $1=i d_{X}$ the family of all subgroups of $G$ of the form

where $F$ is a finite subset of $X$.

$$
S_{F}=\{f \in G:(\forall x \in F) f(x)=x\}
$$

This topology can be described also as the topology induced by the natural embedding of $G$ into the Cartesian power $X^{X}$ equipped with the product topology, where $X$ has the discrete topology.

This topology is also the point-wise convergence topology on $G$. Namely, if $\left(f_{i}\right)_{i \in I}$ is a net in $G$, then $f_{i}$ converges to $f \in G$ precisely when for every $x \in X$ there exists an $i_{0} \in I$ such that for all $i \geq i_{0}$ in $I$ one has $f_{i}(x)=f(x)$.

Exercise 3.14. If $S_{\omega}(X)$ denotes the subset of all permutations of finite support in $S(X)$ prove that $S_{\omega}$ is a dense normal subgroup of $G$.

Exercise 3.15. Prove that $S(X)$ has no proper closed normal subgroups. 


\subsection{Subgroups and quotients of topological groups}

Let $G$ be a topological group and let $H$ be a subgroup of $G$. Then $H$ becomes a topological group when endowed with the topology induced by $G$. Sometimes we refer to this situation by saying $H$ is a topological subgroup of $G$.

Let $G$ and $H$ be topological groups and let $f: G \rightarrow H$ be a continuous homomorphism. If $f$ is simultaneously an isomorphism and a homeomorphism, then $f$ is called a topological isomorphism. If $f: G \rightarrow f(G) \subseteq H$ is a topological isomorphism, where $f(G)$ carries the topology induced by $H$, then $f$ is called topological group embedding, or shortly embedding.

Proposition 3.16. Let $G$ be a topological group and let $H$ be a subgroup of $G$. Then:

(a) $H$ is open in $G$ iff $H$ has a non-empty interior;

(b) if $H$ is open, then $H$ is also closed;

(c) if $H$ is discrete and $G$ is $T_{1}$, then $H$ is closed.

Proof. (a) Let $\emptyset \neq V \subseteq H$ be an open set and let $h_{0} \in V$. Then $1 \in h_{0}^{-1} V \subseteq H=h_{0}^{-1} H$. Now $U=h_{0}^{-1} V$ is open, contains 1 and $h \in h U \subseteq H$ for every $h \in H$. Therefore $H$ is open.

(b) If $H$ is open then every coset $g H$ is open and consequently the complement $G \backslash H$ is open. So $H$ is closed.

(c) Since $H$ is discrete there exists $U \in \mathcal{V}(1)$ with $U \cap H=\{1\}$. Choose $V \in \mathcal{V}(1)$ with $V^{-1} \cdot V \subseteq U$. Then $|x V \cap H| \leq 1$ for every $x \in G$, as $h_{1}=x v_{1} \in x V \cap H$ and $h_{2}=x v_{2} \in x V \cap H$ give $h_{1}^{-1} h_{2} \in V^{-1} \cdot V \cap H=\{1\}$, hence $h_{1}=h_{2}$. Therefore, if $x \notin H$ one can find a neighborhood $W \subseteq x V$ of $x$ with $W \cap H=\emptyset$, i.e., $x \notin \bar{H}$. Indeed, if $x V \cap H=\emptyset$, just take $W=x V$. In case $x V \cap H=\{h\}$ for some $h \in H$, one has $h \neq x$ as $x \notin H$. Then $W=x V \backslash\{x\}$ is the desired neighborhood of $x$.

Exercise 3.17. Let $H$ be a discrete non-trivial group and let $G=H \times N$, where $N$ is an indiscrete non-trivial group. Prove that $H \times\{1\}$ is a discrete non-closed subgroup of $G$.

Let us see now how the closure $\bar{H}$ of a subset $H$ of a topological group $G$ can be computed.

Lemma 3.18. Let $H$ be a subset of $G$. Then with $\mathcal{V}=\mathcal{V}(1)$ one has

(a) $\bar{H}=\bigcap_{U \in \mathcal{V}} U H=\bigcap_{U \in \mathcal{V}} H U=\bigcap_{U, V \in \mathcal{V}} U H V$;

(b) if $H$ is a subgroup of $G$, then $\bar{H}$ is a subgroup of $G$; if $H$ a normal subgroup, then also $\bar{H}$ is normal subgroup;

(c) $N=\overline{\{1\}}$ is a closed normal subgroup.

Proof. (a) For $x \in G$ one has $x \notin \bar{H}$ iff there exists $U \in \mathcal{V}$ such that $x U \cap H=\emptyset=U x \cap H$. Pick a symmetric $U$, i.e., $U=U^{-1}$. Then the latter property is equivalent to $x \notin U H \cup H U$. This proves $\bar{H}=\bigcap_{U \in \mathcal{V}} U H=\bigcap_{U \in \mathcal{V}} H U$. To prove the last equality in (a) note that the already established equalities yield

$$
\bigcap_{U, V \in \mathcal{V}} U H V=\bigcap_{U \in \mathcal{V}}\left(\bigcap_{V \in \mathcal{V}} U H V\right)=\bigcap_{U \in \mathcal{V}} \overline{U H} \subseteq \bigcap_{U \in \mathcal{V}} U^{2} H=\bigcap_{W \in \mathcal{V}} W H=\bar{H} .
$$

(b) Let $x, y \in \bar{H}$. According to (a), to verify $x y \in \bar{H}$ it suffices to see that $x y \in U H U$ for every $U \in \mathcal{V}$. This follows from $x \in U H$ and $y \in H U$ for every $U \in \mathcal{V}$. If $H$ is normal, then for every $a \in G$ and for $U \in \mathcal{V}$ there exists a symmetric $V \in \mathcal{V}$ with $a V \subseteq U a$ and $V a^{-1} \subseteq a^{-1} U$. Now for every $x \in \bar{H}$ one has $x \in V H V^{-1}$, hence $a x a^{-1} \in a V H V^{-1} a^{-1} \subseteq U a H a^{-1} U \subseteq U H U$. This proves $a x a^{-1} \in \bar{H}$ according to (a).

(c) follows from (b) with $H=\{1\}$.

Exercise 3.19. Prove that:

- the subgroup $H \times\{1\}$ from Exercise 3.17 of $G$ is dense.

- for every infinite set $X$ and every group topology on the permutation group $S(X)$ the subgroups $S_{x}=\{f \in$ $S(X): f(x)=x\}, x \in X$, are either closed or dense. (Hint. Prove that $S_{x}$ is a maximal subgroup of $S(X)$, see Fact 3.56.)

Exercise 3.20. Prove that every proper closed subgroup of $\mathbb{R}$ is cyclic. 
(Hint. If $H$ is a proper closed non-trivial subgroup of $\mathbb{R}$ prove that the set $\{h \in H: h>0\}$ has a greatest lower bound $h_{0}$ and conclude that $H=\left\langle h_{0}\right\rangle$.)

Let $G$ be a topological group and $H$ a normal subgroup of $G$. Consider the quotient $G / H$ with the quotient topology, namely the finest topology on $G / H$ that makes the canonical projection $q: G \rightarrow G / H$ continuous. Since we have a group topology on $G$, the quotient topology consists of all sets $q(U)$, where $U$ runs over the family of all open sets of $G$ (as $q^{-1}(q(U))$ is open in $G$ in such a case). In particular, the canonical projection $q$ is open.

The next theorem is due to Frobenius.

Theorem 3.21. If $G$ and $H$ are topological groups, $f: G \rightarrow H$ is a continuous surjective homomorphism and $q: G \rightarrow G / \operatorname{ker} f$ is the canonical homomorphism, then the unique homomorphism $f_{1}: G / \operatorname{ker} f \rightarrow H$, such that $f=f_{1} \circ q$, is a continuous isomorphism. Moreover, $f_{1}$ is a topological isomorphism iff $f$ is open.

Proof. Follows immediately from the definitions of quotient topology and open map.

Independently on its simplicity, this theorem is very important since it produces topological isomorphisms. Openness of the map $f$ is its main ingredient, so from now on we shall be interested in providing conditions that ensure openness (see also $§ 4.1$ ).

Lemma 3.22. Let $X, Y$ be topological spaces and let $\varphi: X \rightarrow Y$ be a continuous open map. Then for every subspace $P$ of $Y$ with $P \cap \varphi(X) \neq \emptyset$ the restriction $\psi: H_{1} \rightarrow P$ of the map $\varphi$ to the subspace $H_{1}=\varphi^{-1}(P)$ is open.

Proof. To see that $\psi$ is open choose a point $x \in H_{1}$ and a neighborhood $U$ of $x$ in $H_{1}$. Then there exists a neighborhood $W$ of $x$ in $X$ such that $U=H_{1} \cap W$. To see that $\psi(U)$ is a neighborhood of $\psi(x)$ in $P$ it suffices to note that if $\varphi(w) \in P$ for $w \in W$, then $w \in H_{1}$, hence $w \in H_{1} \cap W=U$. Therefore $\varphi(W) \cap P \subseteq \varphi(U)=\psi(U)$.

We shall apply this lemma when $X=G$ and $Y=H$ are topological group and $\varphi=q: G \rightarrow H$ is a continuous open homomorphism. Then the restriction $q^{-1}(P) \rightarrow P$ of $q$ is open for every subgroup $P$ of $H$. Nevertheless, even in the particular case when $q$ is surjective, the restriction $H_{1} \rightarrow \varphi\left(H_{1}\right)$ of $q$ to an arbitrary closed subgroup $H_{1}$ of $G$ need not be open.

In the next theorem we see some isomorphisms related the quotient groups.

Teoema 3.23. Let $G$ be a topological group, let $N$ be a normal closed subgroup of $G$ and let $p: G \rightarrow G / N$ be the canonical homomorphism.

(a) If $H$ is a subgroup of $G$, then the homomorphism $i: H N / N \rightarrow p(H)$, defined by $i(x N)=p(x)$, is a topological isomorphism.

(b) If $H$ is a closed normal subgroup of $G$ with $N \subseteq H$, then $p(H)=H / N$ is a closed normal subgroup of $G / N$ and the map $j: G / H \rightarrow(G / N) /(H / N)$, defined by $j(x H)=(x N) .(H / N)$, is a topological isomorphism.

(Both in (a) and (b) the quotient groups are equipped with the quotient topology.)

Proof. (a) As $H N=p^{-1}(p(H))$ we can apply Lemma 3.22 and conclude that $p^{\prime}$ is an open map. Now Theorem 3.21 applies to the restriction $p^{\prime}: H N \rightarrow p(H)$ of $p$.

(b) Since $H=H N$, item (a) implies that the induced topology of $p(H)$ coincides with the quotient topology of $H / N$. Hence we can identify $H / N$ with the topological subgroup $p(H)$ of $G / N$. Since $H=H N$, the set $(G / N) \backslash p(H N)=p(G \backslash H N)$ is open, hence $p(H)$ is closed. Finally note that the composition $f: G \rightarrow$ $(G / N) /(H / N)$ of $p$ with the canonical homomorphism $G / N \rightarrow(G / N) /(H / N)$ is open, being the latter open. Applying to the open homomorphism $f$ with ker $f=H$ Theorem 3.21 we can conclude that $j$ is a topological isomorphism.

Exercise 3.24. Let $G$ be an abelian group equipped with its Bohr topology and let $H$ be a subgroup of $G$. Prove that:

- $H$ is closed in $G$;

- the topological subgroup topology of $H$ coincides with its Bohr topology;

- the quotient topology of $G / H$ coincides with the Bohr topology of $G / H$.

- * G has no convergent sequences [36, §3.4]. 
Exercise 3.25. Let $H$ be a discrete subgroup of a topological group G. Prove that:

- $H \cap \overline{\{1\}}=\{1\}$;

- $\bar{H}$ is isomorphic to the semi-direct product of $H$ and $\overline{\{1\}}$, carrying the product topology, where $H$ is discrete and $\overline{\{1\}}$ is indiscrete.

\subsection{Separation axioms}

Lemma 3.18 easily implies that every topological group is regular, hence:

Proposition 3.26. For a topological group $G$ the following are equivalent:

(a) $G$ is Hausdorff;

(b) $G$ is $T_{0}$.

(c) $G$ is $T_{3}$ (where $T_{3}$ stands for "regular and $T_{1}$ ").

(d) $\overline{\{1\}}=\{1\}$.

A topological group $G$ is monothetic if there exists $x \in G$ with $\langle x\rangle$ dense in $G$.

Exercise 3.27. Prove that:

- a Hausdorff monothetic group is necessarily abelian.

- $\mathbb{T}$ is monothetic.

Is $\mathbb{T}^{2}$ monothetic? What about $\mathbb{T}^{\mathbb{N}}$ ?

Now we relate proprieties of the quotient $G / H$ to those of the subgroup $H$ of $G$.

Lemma 3.28. Let $G$ be a topological group and let $H$ be a normal subgroup of $G$. Then:

(1) the quotient $G / H$ is discrete if and only if $H$ is open;

(2) the quotient $G / H$ is Hausdorff if and only if $H$ is closed.

Let us see now that every $T_{0}$ topological group is also a Tychonov space.

Theorem 3.29. Every Hausdorff topological group is a Tychonov space.

Proof. Let $F$ be a closed set with $1 \notin F$. Then we can find a chain (2) of open neighborhoods of 1 as in Lemma 3.12 such that $F \cap U_{0}=\emptyset$. Let $d$ be the pseudometric defined in Lemma 3.12 and let $f_{F}(x)=d(x, F)$ be the distance function from $F$. This function is continuous in the topology induced by the pseudometric. By the continuity of $d$ it will be continuous also with respect to the topology of $G$. It suffices to note now that $f_{F}(F)=0$, while $f_{F}(1)=1$. This proves that the space $G$ is Tychonov, as the pseudometric is left invariant, so the same argument provides separation of a generic point $a \in G$ from a closed set $F$ that does not contain $a$.

Let $G$ be an abelian group and let $H$ be a family of characters of $G$. Then the characters of $H$ separate the points of $G$ iff for every $x \in G, x \neq 0$, there exists a character $\chi \in H$ with $\chi(x) \neq 1$.

Exercise 3.30. Let $G$ be an abelian group and let $H$ be a family of characters of $G$. Prove that the topology $\mathcal{T}_{H}$ is Hausdorff iff the characters of $H$ separate the points of $G$.

Proposition 3.31. Let $G$ be an infinite abelian group and let $H=\operatorname{Hom}(G, \mathbb{S})$. Then the following holds true:

(a) the characters of $H$ separate the points of $G$,

(b) the Bohr topology $\mathcal{T}_{H}$ is Hausdorff and non-discrete.

Proof. (a) This is Corollary 2.7.

(b) According to Exercise 3.30 item (a) implies that the topology $\mathcal{T}_{H}$ is Hausdorff. Suppose, for a contradiction, that $\mathcal{T}_{H}$ is discrete. Then there exist $\chi_{i} \in H, i=1, \ldots, n$ and $\delta>0$ such that $U\left(\chi_{1}, \ldots, \chi_{n} ; \delta\right)=\{0\}$. In particular, $H=\bigcap_{i=1}^{n} \operatorname{ker} \chi_{i}=\{0\}$. Hence the diagonal homomorphism $f=\chi_{1} \times \ldots \times \chi_{n}: G \rightarrow \mathbb{S}^{n}$ is injective and $f(G) \cong G$ is an infinite discrete subgroup of $\mathbb{S}^{n}$. According to Proposition $3.16 f(G)$ is closed in $\mathbb{S}^{n}$ and consequently, compact. The compact discrete spaces are finite, a contradiction. 
Most often the topological groups in the sequel will be assumed to be Hausdorff.

Example 3.32. Contrary to what we proved in Theorem 3.29 Hausdorff topological groups need not be normal as topological spaces (see Exercise 3.37). A nice "uniform" counter-example to this was given by Trigos: for every uncountable group $G$ the topological group $G^{\#}$ is not normal as a topological space (countable groups are ruled out since every every countable Hausdorff topological group is normal, being a regular Lindelff space).

Theorem 3.33. (Birkhoff-Kakutani) A topological group is metrizable iff it has a countable base of neighborhoods of 1 .

Proof. The necessity is obvious as every point $x$ in a metric space has a countable base of neighborhoods. Suppose now that $G$ has countable base of neighborhoods of 1 . Then one can build a chain (2) of neighborhoods of 1 as in Lemma 3.12 that form a base of $\mathcal{V}(1)$, in particular, $\bigcap_{n=1}^{\infty} U_{n}=\{1\}$. Then the pseudometric produced by the lemma is a metric that induces the topology of the group $G$ because of the inclusions $U_{n} \subseteq B_{1 / n} \subseteq U_{n-1}$.

Exercise 3.34. Prove that subgroups and quotients of metrizable topological groups are metrizable.

Exercise 3.35. Prove that every topological abelian group admits a continuous isomorphism into a product of metrizable abelian groups.

[Hint. For $x \in G, x \neq 0$ choose an open neighborhood $U$ of 0 with $x \in U$. Build a sequence $\left\{U_{n}\right\}$ of symmetric open neighborhoods of 0 with $U_{0} \subseteq U$ and $U_{n}+U_{n} \subseteq U_{n-1}$. Then $H_{U}=\bigcap_{n=1}^{\infty} U_{n}$ is a closed subgroup of $G$.Let $\tau_{U}$ be the group topology on the quotient $G / H_{U}$ having as a local base at 0 the family $\left\{f_{U}\left(U_{n}\right)\right\}$, where $f_{U}: F \rightarrow G / H_{U}$ is the canonical homomorphism. Show that $\left(G / H, \tau_{U}\right)$ is metrizable. Now take the product of all groups $\left(G / H, \tau_{U}\right)$. To conclude observe that the diagonal map of the family $f_{U}$ into the product of all groups $\left(G / H, \tau_{U}\right)$ is continuous and injective. ]

Exercise 3.36. Let $G$ be a Hausdorff topological group. Prove that the centralizer of an element $g \in G$ is a closed subgroup. In particular, the center $Z(G)$ is a closed subgroup of $G$.

Exercise 3.37.* The group $\mathbb{Z}^{\aleph_{1}}$ equipped with the Tychonov topology (where $\mathbb{Z}$ is discrete) is not a normal space [67].

Furstenberg used the natural topology $\nu$ of $\mathbb{Z}$ (see Example 3.7) to find a new proof of the infinitude of prime numbers.

Exercise 3.38. Prove that there are infinitely many primes in $\mathbb{Z}$ using the natural topology $\nu$ of $\mathbb{Z}$.

(Hint. If $p_{1}, p_{2}, \ldots, p_{n}$ were the only primes, then consider the union of the open subgroups $p_{1} \mathbb{Z}, \ldots, p_{n} \mathbb{Z}$ and use the fact that every integer $n \neq 0, \pm 1$ has a prime divisor, so belongs to $\bigcup_{i=1}^{n} p_{i} \mathbb{Z}$.)

\subsection{Connectedness in topological groups}

For a topological group $G$ we denote by $c(G)$ the connected component of 1 and we call it briefly connected component of $G$.

Before proving some basic facts about the connected component, we need an elementary property of the connected sets in a topological groups.

Lemma 3.39. Let $G$ be a topological group.

(a) If $C_{1}, C_{2}, \ldots, C_{n}$ are connected sets in $G$, then also $C_{1} C_{2} \ldots C_{n}$ is connected.

(b) If $C$ is a connected set in $G$, then the set $C^{-1}$ as well as the subgroup generated by $C$ are connected.

Proof. (a) Let us conisder the case $n=2$, the general case easily follows from this one by induction. The subset $C_{1} \times C_{2}$ of $G \times G$ is connected. Now the map $\mu: G \times G \rightarrow G$ defined $\mu(x, y)=x y$ is continuous and $\mu\left(C_{1} \times C_{2}\right)=C_{1} C_{2}$.

(a) For the first part it suffices to note that $C^{-1}$ is a continuous image of $C$ under the continuous map $x \mapsto x^{-1}$.

To prove the second assertion consider the set $C_{1}=C C^{-1}$. It is connected by the previous lemma and obviously $1 \in C_{1}$. Moreover, $C_{1}^{2} \supseteq C \cup C^{-1}$. It remains to note now that the subgroup generated by $C_{1}$ coincides with the subgroup generated by $C$. Since the former is the union of all sets $C_{1}^{n}, n \in \mathbb{N}$ and each set $C_{1}^{n}$ is connected by item (a), we are done. 
Proposition 3.40. The connected component $c(G)$ a topological group $G$ is a closed normal subgroup of $G$. The connected component of an element $x \in G$ is simply the coset $x c(G)=c(G) x$.

Proof. To prove that $c(G)$ is stable under multiplication it suffices to note that $c(G) c(G)$ is still connected (applying item (a) of the above lemma) and contains 1, so must be contained in the connected component $c(G)$. Similarly, an application of item (b) implies that $c(G)$ is stable also w.r.t. the operation $x \mapsto x^{-1}$, so $c(G)$ is a subgroup of $G$. Moreover, for every $a \in G$ the image $a c(G) a^{-1}$ under the conjugation is connected and contains 1 , so must be contains in the connected component $c(G)$. So $c(G)$ is stable also under conjugation. Therefore $c(G)$ is a normal subgroup. The fact that $c(G)$ is closed is well known.

To prove the last assertion it suffices to recall that the maps $y \mapsto x y$ and $y \mapsto y x$ are homeomorphisms.

Our next aim is to see that the quotient $G / c(G)$ is totally disconnected. We need first to see that connectedness and total connectedness are properties stable under extension:

Proposition 3.41. Let $G$ be a topological group and let $N$ be a closed normal subgroup of $G$.

(a) If both $N$ and $G / N$ are connected, then also $G$ is connected.

(b) If both $N$ and $G / N$ are totally disconnected, then also $G$ is totally disconnected.

Proof. Let $q: G \rightarrow G / N$ be the canonical homomorphism.

(a) Let $A \neq \emptyset$ be a clopen set of $G$. As every coset $a N$ is connected, one has either $a N \subseteq A$ or $a N \cap A=\emptyset$. Hence, $A=q^{-1}(q(A))$. This implies that $q(A)$ is a non-empty clopen set of the connected group $G / N$. Thus $q(A)=G / N$. Consequently $A=G$.

(b) Assume $C$ is a connected set in $G$. Then $q(C)$ is a connected set of $G / N$, so by our hypothesis, $q(C)$ is a singleton. This means that $C$ is contained in some coset $x N$. Since $x N$ is totally disconnected as well, we conclude that $C$ is a singleton. This proves that $G$ is totally disconnected.

Lemma 3.42. If $G$ is a topological group, then the group $G / c(G)$ is totally disconnected.

Proof. Let $q: G \rightarrow G / c(G)$ be the canonical homomorphism and let $H$ be the inverse image of $c(G / c(G))$ under $q$. Now apply Proposition 3.41 to the group $H$ and the quotient group $H / c(G) \cong c(G / c(G))$ to conclude that $H$ is connected. Since it contains $c(G)$, we have $H=c(G)$. Hence $G / c(G)$ is totally disconnected.

For a topological group $G$ denote by $Q(G)$ the quasi-component of the neutral element 1 of $G$ (i.e., the intersection of all clopen sets of $G$ containing 1 ) and call it quasi-component of $G$.

Proposition 3.43. For a topological group $G$ the quasi-component $Q(G)$ is a closed normal subgroup of $G$. The quasi-component of $x \in G$ coincides with the coset $x Q(G)=Q(G) x$.

Proof. Let $x, y \in Q(G)$. To prove that $x y \in Q(G)$ we need to verify that $x y \in O$ for every clopen set $O$ containing 1 . Let $O$ be such a set, then $x, y \in O$. Obviously $O y^{-1}$ is a clopen set containing 1 , hence $x \in O y^{-1}$. This implies $x y \in O$. Hence $Q(G)$ is stable under multiplication. For every clopen set $O$ containing 1 the set $O^{-1}$ has the same propriety, hence $Q(G)$ is stable also w.r.t. the operation $a \mapsto a^{-1}$. This implies that $Q(G)$ is a subgroup. Moreover, for every $a \in G$ and for every clopen set $O$ containing 1 also its image $a O a^{-1}$ under the conjugation is a clopen set containing 1 . So $Q(G)$ is stable also under conjugation. Therefore $Q(G)$ is a normal subgroup. Finally, as an intersection of closen sets, $Q(G)$ is closed.

Remark 3.44. It follows from Lemma 2.14 that $c(G)=Q(G)$ for every compact topological group $G$. Actually, this remains true also in the case of locally compact groups $G$ (cf. 4.22).

In the next remark we discuss zero-dimensionality.

Remark 3.45. (a) It follows immediately from Proposition 3.16 that every linear group topology is zerodimensional; in particular, totally disconnected.

(b) Every countable Hausdorff topological group is zero-dimensional (this is true for topological spaces as well).

We shall see in the sequel that for locally compact abelian groups or compact groups the implication from item (a) can be inverted (see Theorem 4.18). On the other hand, the next example shows that local connectedness is essential.

Example 3.46. The group $\mathbb{Q} / \mathbb{Z}$ is zero-dimensional but has no proper open subgroups. 


\subsection{Group topologies determined by sequences}

Let $G$ be an abelian group and let $\left(a_{n}\right)$ be a sequence in $G$. The question of the existence of a Hausdorff group topology that makes the sequence $\left(a_{n}\right)$ converge to 0 is not only a mere curiosity. Indeed, assume that some Hausdorff group topology $\tau$ makes the sequence $\left(p_{n}\right)$ of all primes converge to zero. Then $p_{n} \rightarrow 0$ would yield $p_{n}-p_{n+1} \rightarrow 0$ in $\tau$, so this sequence cannot contain infinitely many entries equal to 2 . This would provide a very easy negative solution to the celebrated problem of the infinitude of twin primes (actually this argument would show that the shortest distance between two consecutive primes converges to $\infty$ ).

Definition 3.47. [89] A sequence $A=\left\{a_{n}\right\}_{n}$ in an abelian group $G$ is called a $T$-sequence is there exists a Hausdorff group topology on $G$ such that $a_{n} \rightarrow 0 .^{2}$

Let $\left(a_{n}\right)$ be a T-sequence in an abelian group $G$. Hence the family $\left\{\tau_{i}: i \in I\right\}$ of Hausdorff group topologies on the group $G$ such that $a_{n} \rightarrow 0$ in $\tau_{i}$ is non-empty. Let $\tau=\sup _{i \in I} \tau_{i}$, then by Exercise $3.5 a_{n} \rightarrow 0$ in $\tau$ as well. Clearly, this is the finest group topology in which $a_{n}$ converges to 0 . This is why we denote it by $\tau_{A}$ or $\tau_{\left(a_{n}\right) \cdot{ }^{3}}$

Before discussing the topology $\tau_{\left(a_{n}\right)}$ and how $T$-sequences can be described in general we consider a couple of examples:

Example 3.48. (a) Let us see that the sequences $\left(n^{2}\right)$ and $\left(n^{3}\right)$ are not a $T$-sequence in $\mathbb{Z}$. Indeed, suppose for a contradiction that some Hausdorff group topology $\tau$ on $\mathbb{Z}$ makes $n^{2}$ converge to 0 . Then $(n+1)^{2}$ converges to 0 as well. Taking the difference we conclude that $2 n+1$ converges to 0 as well. Since obviously also $2 n+3$ converges to 0 , we conclude, after substraction, that the constant sequence 2 converges to 0 . This is a contradiction, since $\tau$ is Hausdroff. We leave the case $\left(n^{3}\right)$ as an exercise to the reader.

(b) A similar argument proves that the sequence $P_{d}(n)$, where $P_{d}(x) \in \mathbb{Z}[x]$ is a fixed polynomial with $\operatorname{deg} P_{d}=d>0$, is not a $T$-sequence in $\mathbb{Z}$.

Protasov and Zelenyuk [88] established a number of nice properties of the finest group topology $\tau_{\left(a_{n}\right)}$ on $G$ that makes $\left(a_{n}\right)$ converge to 0 .

For an abelian group $G$ and subsets $A_{1}, \ldots, A_{n} \ldots$ of $G$ we denote by $\pm A_{1} \pm \ldots \pm A_{n}$ the set of all sums $g=g_{1}+\ldots+g_{n}$, where $g_{i} \in\{0\} \cup A_{i} \cup-A_{i}$ for every $i=1, \ldots, n$. Let

$$
\pm A_{1} \pm \ldots \pm A_{n} \pm \ldots=\bigcup_{n=1}^{\infty} \pm A_{1} \pm \ldots \pm A_{n} .
$$

If $A=\left\{a_{n}\right\}_{n}$ is a sequence in $G$, for $m \in \mathbb{N}$ denote by $A_{m}$ the "tail" $\left\{a_{m}, a_{m+1}, \ldots\right\}$. For $k \in \mathbb{N}$ let $A(k, m)= \pm A_{m} \pm \ldots \pm A_{m}$ ( $k$ times).

Remark 3.49. The existence of a finest group topology $\tau_{A}$ on an abelian group $G$ that makes an arbitrary given sequence $A=\left\{a_{n}\right\}_{n}$ in $G$ converge to 0 is easy to prove as far as we are not interested on imposing the Hausdorff axiom. Indeed, as $a_{n}$ converges to 0 in the indiscrete topology, $\tau_{A}$ is simply the supremum of all group topologies $\tau$ on $G$ such that $a_{n}$ converges to 0 in $\tau$. This gives no idea on how this topology looks like. One can easily describe it as follows.

Let $m_{1}, \ldots, m_{n}, \ldots$ be a sequence of natural numbers. Denote by $A\left(m_{1}, \ldots, m_{n}, \ldots\right)$ the set

$$
\pm A_{m_{1}} \pm \ldots \pm A_{m_{n}} \pm \ldots
$$

and by $\mathcal{B}_{A}$ the family of all sets $A\left(m_{1}, \ldots, m_{n}, \ldots\right)$ when $m_{1}, \ldots, m_{n}, \ldots$ vary in $\mathbb{N}^{\mathbb{N}}$. Then $\mathcal{B}_{A}$ is a filter base, satisfying the axioms of group topology. The group topology $\tau$ defined in this way satisfies the required conditions. Indeed, obviously $a_{n} \rightarrow 0$ in $(G, \tau)$ and $\tau$ contains any other group topology with this property. Consequently, $\tau=\tau_{A}$.

Note that

$$
A(k, m) \subseteq A\left(m_{1}, \ldots, m_{n}, \ldots\right),
$$

for every $k \in \mathbb{N}$, where $m=\max \left\{m_{1}, \ldots, m_{k}\right\}$. The sets $A(k, m)$, for $k, m \in \mathbb{N}$, form a filter base, but the filter they generate need not be the filter of neighborhoods of 0 in a group topology. The utility of this family becomes clear now.

\footnotetext{
${ }^{2}$ We shall see below that the sequence $\left(p_{n}\right)$ of all primes is not a $T$-sequence in the group $\mathbb{Z}$ (see Exercise 4.32 ). So the above mentioned possibility to resolve the problem of the infinitude of twin primes does not work.

${ }^{3}$ To simplify things we consider only sequences without repetition, hence the convergence to zero $a_{n} \rightarrow 0$ depends only on the set $A=\left\{a_{n}\right\}_{n}$, it does not depend on the enumeration of the sequence.
} 
Theorem 3.50. A sequence $A=\left\{a_{n}\right\}_{n}$ in an abelian group $G$ is a T-sequence iff

$$
\bigcap_{m=1}^{\infty} A(k, m)=0 \text { for every } k \in \mathbb{N} .
$$

Proof. Obviously the sequence $A=\left\{a_{n}\right\}_{n}$ is a T-sequence iff the topology $\tau_{A}$ is Hausdorff. Clearly, $\tau_{A}$ is Hausdorff iff $\bigcap_{m_{1}, \ldots, m_{n}, \ldots}^{\infty} A\left(m_{1}, \ldots, m_{n}, \ldots\right)=0$. If $\tau_{A}$ is Hausdorff, then (2) holds by (1). It remains to see that (2) implies $\bigcap_{m_{1}, \ldots, m_{n}, \ldots}^{\infty} A\left(m_{1}, \ldots, m_{n}, \ldots\right)=0$. First of all note that $A\left(m_{1}, \ldots, m_{n}, \ldots\right) \supseteq A\left(m_{1}^{*}, \ldots, m_{n}^{*}, \ldots\right)$, where $m_{n}^{*}=\max \left\{m_{1}, \ldots, m_{n}\right\}$. Moreover, the sequence $\left(m_{n}^{*}\right)$ is increasing. Hence

$$
\bigcap_{m_{1}, \ldots, m_{n}, \ldots}^{\infty} A\left(m_{1}, \ldots, m_{n}, \ldots\right)=\bigcap_{m_{1}^{*}, \ldots, m_{n}^{*}, \ldots}^{\infty} A\left(m_{1}^{*}, \ldots, m_{n}^{*}, \ldots\right)
$$

where the second intersection is taken only over the increasing sequences $\left(m_{n}^{*}\right)$. Obviously, for every increasing sequence $\left(m_{n}^{*}\right)$ one has

$$
A\left(m_{1}^{*}, \ldots, m_{n}^{*}, \ldots\right) \subseteq \bigcup_{k=1}^{\infty} A\left(k, m_{1}^{*}\right)
$$

This yields

$$
\bigcap_{m_{1}^{*}, \ldots, m_{n}^{*}, \ldots}^{\infty} A\left(m_{1}^{*}, \ldots, m_{n}^{*}, \ldots\right) \subseteq \bigcap_{m_{1}^{*}=1}^{\infty} \bigcup_{k=1}^{\infty} A\left(k, m_{1}^{*}\right)=\bigcup_{k=1}^{\infty} \bigcap_{m_{1}^{*}=1}^{\infty} A\left(k, m_{1}^{*}\right)=0
$$

Since every infinite abelian group $G$ admits a non-discrete metrizable group topology, there exist non-trivial (i.e., having all members non-zero) $T$-sequences.

A notion similar to $T$-sequence, but defined with respect to only topologies induced by characters, will be given in $\S 6.2$. From many points of view it turns out to be easier to deal with than $T$-sequence. In particular, we shall see easy sufficient condition for a sequence of integers to be a $T$-sequence.

We give without proof the following technical lemma that will be useful in $\S 6.2$.

Lemma 3.51. [89] For every $T$-sequence $A=\left\{a_{n}\right\}$ in $\mathbb{Z}$ there exists a sequence $\left\{b_{n}\right\}$ in $\mathbb{Z}$ such that for every choice of the sequence $\left(e_{n}\right)$, where $e_{n} \in\{0,1\}$, the sequence $q_{n}$ defined by $q_{2 n}=b_{n}+e_{n}$ and $q_{2 n-1}=a_{n}$, is a T-sequence.

Exercise 3.52. (a) Prove that there exists a T-sequence $\left(a_{n}\right)$ in $\mathbb{Z}$ with $\lim _{n} \frac{a_{n+1}}{a_{n}}=1$ [89] (see also Example 6.12).

$(b)^{*}$ Every sequence $\left(a_{n}\right)$ in $\mathbb{Z}$ with $\lim _{n} \frac{a_{n+1}}{a_{n}}=+\infty$ is a T-sequence [89, 7] (see Theorem 6.11).

(c)* Every sequence $\left(a_{n}\right)$ in $\mathbb{Z}$ such that $\lim _{n} \frac{a_{n+1}}{a_{n}} \in \mathbb{R}$ is transcendental is a T-sequence [89].

\subsection{Markov's problems}

\subsubsection{The Zariski topology and the Markov topology}

Let $G$ be a Hausdorff topological group, $a \in G$ and $n \in \mathbb{N}$. Then the set $\left\{x \in G: x^{n}=a\right\}$ is obviously closed in $G$. This simple fact motivated the following notions due to to Markov [76].

A subset $S$ of a group $G$ is called:

(a) elementary algebraic if there exist an integer $n>0, a_{1}, \ldots, a_{n} \in G$ and $\varepsilon_{1}, \ldots, \varepsilon_{n} \in\{-1,1\}$ such that

$$
S=\left\{x \in G: x^{\varepsilon_{1}} a_{1} x^{\varepsilon_{2}} a_{2} \ldots a_{n-1} x^{\varepsilon_{n}}=a_{n}\right\}
$$

(b) algebraic if $S$ is an intersection of finite unions of elementary algebraic subsets,

(c) unconditionally closed if $S$ is closed in every Hausdorff group topology of $G$. 
Since the family of all finite unions of elementary algebraic subsets is closed under finite unions and contains all finite sets, it is a base of closed sets of some $T_{1}$ topology $\mathfrak{Z}_{G}$ on $G$, called the Zariski topology ${ }^{4}$. Clearly, the $\mathfrak{Z}_{G}$-clsoed sets are precisely the algebraic sets in $G$.

Analogously, the family of all unconditionally closed subsets of $G$ coincides with the family of closed subsets of a $T_{1}$ topology $\mathfrak{M}_{G}$ on $G$, namely the infimum (taken in the lattice of all topologies on $G$ ) of all Hausdorff group topologies on $G$. We call $\mathfrak{M}_{G}$ the Markov topology of $G$. Note that $\left(G, \mathfrak{Z}_{G}\right)$ and $\left(G, \mathfrak{M}_{G}\right)$ are quasi-topological groups, i.e., the inversion and translations are continuous. Nevertheless, when $G$ is abelian $\left(G, \mathfrak{Z}_{G}\right)$ and $\left(G, \mathfrak{M}_{G}\right)$ are not group topologies unless they are discrete.

Since an elementary algebraic set of $G$ must be closed in every Hausdorff group topology on $G$, one always has $\mathfrak{Z}_{G} \subseteq \mathfrak{M}_{G}$. In 1944 Markov [76] asked if the equality $\mathfrak{Z}_{G}=\mathfrak{M}_{G}$ holds for every group $G$. He himself showed that the answer is positive in case $G$ is countable [76]. Moreover, in the same manuscript Markov attributes to Perel'man the fact that $\mathfrak{Z}_{G}=\mathfrak{M}_{G}$ for every Abelian group $G$ (a proof has never appeared in print until [37]). An example of a group $G$ with $\mathfrak{Z}_{G} \neq \mathfrak{M}_{G}$ was given by Gerchard Hesse [66].

Exercise 3.53. Show that if $(G, \cdot)$ is an abelian group, then every elementary algebraic set of $G$ has the form $\left\{x \in G: x^{n}=a\right\}, a \in G$.

\subsubsection{The Markov topology of the symmetric group}

Let $X$ be an infinite set. In the sequel we denote by $\tau_{X}$ the pointwise convergence topology of the infinite symmetric group $S(X)$ defined in $§ 3.2 .4$. It turns out that the Markov topology of $S(X)$ coincides with $\tau_{X}$ :

Theorem 3.54. Then Markov topology on $S(X)$ coincides with the topology $\tau_{X}$ of pointwise convergence of $S(X)$.

This theorem follows immediately from the following old result due to Gaughan.

Theorem 3.55. ([36]) Every Hausdorff group topology of the infinite permutation group $S(X)$ contains the topology $\tau_{X}$.

The proof of this theorem follows more or less the line of the proof exposed in [36, $\S 7.1]$ with several simplifications. The final stage of the proof is preceded by a number of claims (and their corollaries) and two facts about purely algebraic properties of the group $S(X)$ (3.56 and 3.59). The claims and their corollaries are given with complete proofs. To give an idea about the proofs of the two algebraic facts, we prove the first one; the proof of the second one can be found in [36, Lemmas 7.1.4, 7.1.8] (actually, only a fragment of the proof of [36, Lemmas 7.1.8] is needed for the proof of item (b) of Fact 3.59).

We say for a subset $A$ of $S(X)$ that $A$ is $m$-transitive for some positive integer $m$ if for every $Y \subseteq X$ of size at most $m$ and every injection $f: Y \rightarrow X$ there exists $a \in A$ that extends $f$. ${ }^{5}$ The leading idea is that a transitive subset $A$ of $S(X)$ is placed "generically" in $S(X)$, whereas a non-tranisitve one is a subset of some subgroup of $S(X)$ that is a direct product $S(Y) \times S(X \backslash Y)$. (Here and in the sequel, for a subset $Y$ of $X$ we tacitly identify the group $S(Y)$ with the subgroup of $S(X)$ consisting of all permutations of $S(X)$ that are identical on $X \backslash Y$.)

The first fact concerns the stabilizers $S_{x}=S_{\{x\}}=\{f \in S(X): f(x)=x\}$ of points $x \in X$. They consitute a prebase of the filter of neighborhoods of $i d_{X}$ in $\tau_{X}$.

Fact 3.56. For every $x \in X$ the subgroup $S_{x}$ of $S(X)$ is maximal.

Proof. Assume $H$ is a subgroup of $S(X)$ properly containing $S_{x}$. To show that $H=S(X)$ take any $f \in S(X)$. If $y=f(x)$ coincides with $x$, then $f \in S_{x} \subseteq H$ and we are done. Assume $y \neq x$. Get $h \in H \backslash S_{x}$. Then $z=h(x) \neq x$, so $x \notin\{z, y\}$. There exists $g \in S(X)$ such that $g(x)=x, g(y)=z$ and $g(z)=y$. Then $g \in S_{x} \subseteq H$ and $f(x)=g(h(x))=y$, so $h^{-1} g^{-1} f(x)=x$ and $h^{-1} g^{-1} f \in S_{x} \cap G \subseteq H$. So $f \in g h H=H$.

Claim 3.57. Let $T$ be a Hausdorff group topology on $S(X)$. If a subgroups of $S(X)$ of the form $S_{x}$ is $T$-closed, then it is also T-open.

Proof. As $S_{x}$ is $T$-closed, for every fixed $y \neq x$ the set $V_{y}=\{f \in S(X): f(x) \neq y\}$ is $T$-open and contains 1 . So there exists a symmetric neighborhood $W$ of 1 in $T$ such that $W . W \subseteq V_{y}$. By the definition of $V_{y}$ this gives $W x \cap W y=\emptyset$. Then either $|X \backslash W x|=|X|$ or $|X \backslash W y|=|X|$. Suppose this occurs with $x$, i.e., $|X \backslash W x|=|X|$.

\footnotetext{
${ }^{4}$ Some authors call it also the verbal topology [20], we prefer here Zariski topology coined by most authors [10].

${ }^{5}$ Note that a countable subset $H$ of $S(X)$ cannot be transitive unless $X$ itself is countable.
} 
Then one can find a permutation $f \in S(X)$ that sends $W x \backslash\{x\}$ to the complement of $W x$ and $f(x)=x$. Such an $f$ satisfies:

$$
f W f^{-1} \cap W \subseteq S_{x}
$$

as $f W f^{-1}(x)$ meets $W x$ precisely in the singleton $\{x\}$ by the choice of $f$. This proves that $S_{x}$ is $T$-open.

Analogous argument works for $S_{y}$ when $|X \backslash W y|=|X|$.

Corollary 3.58. If $T$ be a Hausdorff group topology on $S(X)$ that does not contain $\tau_{X}$, then all subgroups of $S(X)$ of the form $S_{x}$ are $T$-dense.

Proof. Since the subgroups $S_{x}$ of $S(X)$ form a prebase of the filter of neighborhoods of $i d_{X}$ in $S(X)$, out hypothesis implies that some subgroup $S_{x}$ is not $T$-open. By Claim $3.57 S_{x}$ is not $T$-closed either. By Fact $3.56 S_{x}$ is $T$-dense. Since all subgroups of the form $S_{y}$ are conjugated, this implies that stabilizers $S_{y}$ are $T$-dense.

This was the first step in the proof. The next step will be establishing that $S_{x, y}$ are never dense in any Hausdorff group topology on $S(X)$ (Corollary 3.62).

In the sequel we need the subgroup $\widetilde{S}_{x, y}:=S_{x, y} \times S(\{x, y\})$ of $S(X)$ that contains $S_{x, y}$ as a subgroup of index 2. Note that $\widetilde{S}_{x, y}$ is precisely the subgroup of all permutations in $S(X)$ that leave the doubleton $\{x, y\}$ set-wise invariant.

Fact 3.59. For any doubleton $x, y$ in $X$ the following holds true:

(a) the subgroup $\widetilde{S}_{x, y}$ of $S(X)$ is maximal;

(b) every proper subgroup of $S(X)$ properly containing $S_{x, y}$ coincides with one of the subgroups $S_{x}, S_{y}$ or $\widetilde{S}_{x, y}$.

Claim 3.60. Let $T$ be a Hausdroff group topology on $S(X)$, then there exists a $T$-nbd of 1 that is not 2-transitive.

Proof. Assume for a contradiction that all $T$-neighborhoods of $i d_{X}$ that are 2-transitive. Fix distinct $u, v, w \in$ $X$. We show now that the 3-cycle $(u, v, w) \in V$ for every arbitrarily fixed $T$-neighborhood of $i d_{X}$. Indeed, choose a symmetric $T$-neighborhood $W$ of $i d_{X}$ such that $W^{2} \subseteq V$. Let $f$ be the transposition (uv). Then $U=f W f \cap W \in T$ is a neighborhood of 1 and $f U f=U$. Since $U$ is 2-transitive there exists $g \in U$ such that $g(u)=u$ and $g(v)=w$. Then $(u, v, w)=g f g^{-1} f \in W \cdot(f U f) \subseteq W^{2} \subseteq V$.

Claim 3.61. Let $T$ be a group topology on $S(X)$. Then

(a) every $T$-nbd $V$ of $i d_{X}$ in $S(X)$ is transitive iff every stabilizer $S_{x}$ is $T$-dense;

(b) every $T$-nbd $V$ of $i d_{X}$ in $S(X)$ is m-transitive iff every stabilizer $S_{F}$ with $|F| \leq m$ is $T$-dense.

Proof. Assume that some (hence all) $S_{z}$ is $T$-dense in $S(X)$. To prove that $V$ is transitive consider a pair $x, y \in X$. Let $t=(x y)$. By the $T$-density of $S_{x}$ the $T$-nbd $t^{-1} V$ of $t^{-1}$ meets $S_{x}$, i.e., for some $v \in V$ one has $t^{-1} v \in S_{x}$. Then $v \in t S_{x}$ obviously satisfies $v x=y$.

A similar argument proves that transitivity of each T-nbd of 1 entails that every stabilizer $S_{x}$ is $T$-dense.

(b) The proof in the case $m>1$ is similar.

What we really need further on (in particular, in the next corollary) is that the density of the stabilizers $S_{x, y}$ imply that every $T$-nbd $V$ of $i d_{X}$ in $S(X)$ is 2-transitive.

Corollary 3.62. Let $T$ be a Hausdroff group topology on $S(X)$. Then $S_{x, y}$ is $T$-dense for no pair $x, y$ in $X$.

Proof. Follows from claims 3.60 and 3.61

Proof of Theorem 3.55. Assume for a contradiction that $T$ is a Hausdroff group topology on $S(X)$ that does not contain $\tau_{X}$. Then by corollaries 3.58 and 3.62 all subgroups of the form $S_{x}$ are $T$-dense and no subgroup of the form $S_{x, y}$ is $T$-dense. Now fix a pair $x, y \in X$ and let $G_{x, y}$ denote the $T$-closure of $S_{x, y}$. Then $G_{x, y}$ is a proper subgroup of $S(X)$ containing $S_{x, y}$. Since $S_{x}$ is dense, $G_{x, y}$ cannot contain $S_{x}$, so $S_{x} \cap G_{x, y}$ is a proper subgroup of $S_{x}$ containing $S_{x, y}$. By Claim 3.56 applied to $S_{x}=S(X \backslash\{x\})$ and its subgroup $S_{x, y}$ (the stabilizer of $y$ in $S_{x}$ ), we conclude that $S_{x, y}$ is a maximal subgroup of $S_{x}$. Therefore, $S_{x} \cap G_{x, y}=S_{x, y}$. This shows that $S_{x, y}$ is a $T$-closed subgroup of $S_{x}$. By Claim 3.57 applied to $S_{x}=S(X \backslash\{x\})$ and its subgroup $S_{x, y}$, we conclude that $S_{x, y}$ is a $T$-open subgroup of $S_{x}$. Since $S_{x}$ is dense in $S(X)$, we can claim that $G_{x, y}$ is a $T$-open subgroup of $S(X)$. Since $S_{x}$ is a proper dense subgroup of $S(X)$, it is clear that $S_{x}$ cannot contain $G_{x, y}$. Analogously, $S_{y}$ 
cannot contain $G_{x, y}$ either. So $G_{x, y} \neq S_{x, y}$ is a proper subgroup of $S(X)$ containing $S_{x, y}$ that does not coincide with $S_{x}$ or $S_{y}$. Therefore $G_{x, y}=\widetilde{S}_{x, y}$ by Fact 3.59 . This proves that $\widetilde{S}_{x, y}$ is $T$-open. Since all subgroups of the form $\widetilde{S}_{x, y}$ are pairwise conjugated, we can claim that all subgroups $\widetilde{S}_{x, y}$ is $T$-open.

Now we can see that the stabilizers $S_{F}$ with $|F|>2$ are T-open, as

$$
S_{F}=\bigcap\left\{\widetilde{S}_{x, y}: x, y \in F, x \neq y\right\} .
$$

This proves that all basic neighborhoods $S_{F}$ of 1 in $\tau_{X}$ are $T$-open. In particular, also the subgroups $S_{x}$ are $T$-open, contrary to our hypothesis.

\subsubsection{Existence of Hausdorff group topologies}

According to Proposition 3.31 every infinite abelian group admits a non-discrete Hausdorff group topology, for example the Bohr topology. This gives immediately the following

Corollary 3.63. Every group with infinite center admits a non-discrete Hausdorff group topology.

Proof. The center $Z(G)$ of the group $G$ has a non-discrete Hausdorff group topology $\tau$ by the above remark. Now consider the family $\mathcal{B}$ of all sets of the form $a U$, where $a \in G$ and $U$ is a non-empty $\tau$-subset of $Z(G)$. It is easy to see that it is a base of a non-discrete Hausdorff group topology on $G$.

In 1946 Markov set the problem of the existence of a (countably) infinite group $G$ that admits no Hausdorff group topology beyond the discrete one. Let us call such a group a Markov group. Obviously, $G$ is a Markov group precisely when $\mathfrak{M}_{G}$ is discrete. A Markov group must have finite center by Corollary 3.63.

According to Proposition 3.26, the closure of the neutral element of every topological group is always a normal subgroup of $G$. Therefore, a simple topological group is either Hausdorff, or indiscrete. So a simple Markov group $G$ admits only two group topologies, the discrete and the indiscrete ones.

The equality $\mathfrak{Z}_{G}=\mathfrak{M}_{G}$ established by Markov in the countable case was intended to help in finding a countably infinite Markov group $G$. Indeed, a countable group $G$ is Markov precisely when $\mathfrak{Z}_{G}$ is discrete. Nevertheless, Markov failed in building a countable group $G$ with discrete Zariski topology; this was done much later, in 1980, by Ol'shanskii [78] who made use of the so called Adian groups $A=A(m, n)$ (constructed by Adian to negatively resolve the famous 1902 Burnside problem on finitely generated groups of finite exponent). Let us sketch here Ol'shanskii's elegant short proof.

Example 3.64. [78] Let $m$ and $n$ be odd integers $\geq 665$, and let $A=A(m, n)$ be Adian's group having the following properties

(a) $A$ is generated by $n$-elements;

(b) $A$ is torsion-free;

(c) the center $C$ of $A$ is infinite cyclic.

(d) the quotient $A / C$ is infinite, of exponent $m$, i.e., $y^{m} \in C$ for every $y \in A .^{6}$

By (a) the group $A$ is countable. Denote by $C^{m}$ the subgroup $\left\{c^{m}: c \in C\right\}$ of $A$. Let us see that (b), (c) and (d) jointly imply that the Zariski topology of the infinite quotient $G=A / C^{m}$ is discrete (so $G$ is a countably infinite Markov group). Let $d$ be a generator of $C$. Then for every $x \in A \backslash C$ one has $x^{m} \in C \backslash C^{m}$. Indeed, if $x^{m}=d^{m s}$, then $\left(x d^{-s}\right)^{m}=1$ for some $s \in \mathbb{Z}$, so $x d^{-s}=1$ and $x \in C$ by (b). Hence

for every $u \in G \backslash\{1\}$ there exists $a \in C \backslash C^{m}$, such that either $u=a$ or $u^{m}=a$.

As $\left|C / C^{m}\right|=m$, every $u \in G \backslash\{1\}$ is a solution of some of the $2(m-1)$ equations in (3). Thus, $G \backslash\{e\}$ is closed in the Zariski topology $\mathfrak{Z}_{G}$ of $G$. Therefore, $\mathfrak{Z}_{G}$ is discrete.

Now we recall an example, due to Shelah [92], of an uncountable group which is non-topologizable. It appeared about a year or two earlier than the ZFC-example of Ol'shanskii exposed above.

Example 3.65. [92] Under the assumption of $\mathrm{CH}$ there exists a group $G$ of size $\omega_{1}$ satisfying the following conditions (a) (with $m=10000$ ) and (b) (with $n=2$ ):

(a) there exists $m \in \mathbb{N}$ such that $A^{m}=G$ for every subset $A$ of $G$ with $|A|=|G|$;

\footnotetext{
${ }^{6}$ i.e., the finitely generated infinite quotient $A / C$ negatively resolves Burnside's problem.
} 
(b) for every subgroup $H$ of $G$ with $|H|<|G|$ there exist $n \in \mathbb{N}$ and $x_{1}, \ldots, x_{n} \in G$ such that the intersection $\bigcap_{i=1}^{n} x_{i}^{-1} H x_{i}$ is finite.

Let us see that $G$ is a Markov group (i.e., $\mathfrak{M}_{G}$ is discrete) $)^{7}$. Assume $\mathcal{T}$ be a Hausdorff group topology on $G$. There exists a $\mathcal{T}$-neighbourhood $V$ of $e_{G}$ with $V \neq G$. Choose a $\mathcal{T}$-neighbourhood $W$ of $e_{G}$ with $W^{m} \subseteq V$. Now $V \neq G$ and (a) yield $|W|<|G|$. Let $H=\langle W\rangle$. Then $|H|=|W| \cdot \omega<|G|$. By (b) the intersection $O=\bigcap_{i=1}^{n} x_{i}^{-1} H x_{i}$ is finite for some $n \in \mathbb{N}$ and elements $x_{1}, \ldots, x_{n} \in G$. Since each $x_{i}^{-1} H x_{i}$ is a $\mathcal{T}$-neighbourhood of $e_{G}$, this proves that $e_{G} \in O \in \mathcal{T}$. Since $\mathcal{T}$ is Hausdorff, it follows that $\left\{e_{G}\right\}$ is $\mathcal{T}$-open, and therefore $\mathcal{T}$ is discrete.

One can see that even the weaker form of (a) (with $m$ depending on $A \in[G]^{|G|}$ ), yields that every proper subgroup of $G$ has size $<|G|$. In the case $|G|=\omega_{1}$, the groups with this property are known as Kurosh groups (in particular, this is a Jonsson semigroup of size $\omega_{1}$, i.e., an uncountable semigroup whose proper subsemigroups are countable).

Finally, this remarkable construction from [92] furnished also the first consistent example to a third open problem. Namely, a closer look at the above argument shows that the group $G$ is simple. As $G$ has no maximal subgroups, it shows also that taking Frattini subgroup " "does not commute" with taking finite direct products (indeed, $\operatorname{Fratt}(G)=G$, while $\operatorname{Fratt}(G \times G)=\Delta_{G}$ the "diagonal" subgroup of $G \times G$ ).

\subsubsection{Extension of group topologies}

The problem of the existence of (Hausdorff non-discrete) group topologies can be considered also as a problem of extension of (Hausdorff non-discrete) group topologies.

The theory of extension of topological spaces is well understood. If a subset $Y$ of a set $X$ carries a topology $\tau$, then it is easy to extend $\tau$ to a topology $\tau^{*}$ on $X$ such that $(Y, \tau)$ is a subspace of $\left(X, \tau^{*}\right)$. The easiest way to do it is to consider $X=Y \cup(X \backslash Y)$ as a partition of the new space $\left(X, \tau^{*}\right)$ into clopen sets and define the topology of $X \backslash Y$ arbitrarily. Usually, one prefers to define the extension topology $\tau^{*}$ on $X$ in such a way to have $Y$ dense in $X$. In such a case the extensions of a given space $(Y, \tau)$ can be described by means of appropriate families of open filters of $Y$ (i.e., filters on $Y$ having a base of $\tau$-open sets).

The counterpart of this problem for groups and group topologies is much more complicated because of the presence of group structure. Indeed, let $H$ be a subgroup of a group $G$ and assume that $\tau$ is a group topology of $H$. Now one has to build a group topology $\tau^{*}$ on $G$ such that $(H, \tau)$ is a topological subgroup of $\left(G, \tau^{*}\right)$. The first idea to extend $\tau$ is to imitate the first case of extension considered above by declaring the subgroup $H$ a $\tau^{*}$-open topological subgroup of the new topological group $\left(G, \tau^{*}\right)$. Let us note that this would immediately determine the topology $\tau^{*}$ in a unique way. Indeed, every coset $g H$ of $H$ must carry the topology transported from $H$ to $g H$ by the translation $x \mapsto g x$, i.e., the $\tau^{*}$-open subsets of $g H$ must have the form $g U$, where $U$ is an open subset of $(H, \tau)$. In other words, the family $\{g U: \emptyset \neq U \in \tau\}$ is a base of $\tau^{*}$. This idea has worked in the proof of Corollary 3.63 where $H$ was the center of $G$. Indeed, this idea works in the following more general case.

Lemma 3.66. Let $H$ be a subgroup of a group $G$ such that $G=H c_{G}(G)$. Then for every group topology $\tau$ on $H$ the above described topology $\tau^{*}$ is a group topology of $G$ such that $(H, \tau)$ is a topological subgroup of $\left(G, \tau^{*}\right)$.

Proof. The first two axioms on the neighborhood base are easy to check. For the third one pick a basic $\tau^{*}-$ neighborhood $U$ of 1 in $G$. Since $H$ is $\tau^{*}$-open, we can assume wlog that $U \subseteq H$, so $U$ is a $\tau$-neighborhood of 1. Let $x \in G$. We have to produce a $\tau^{*}$-neighborhood $V$ of 1 in $G$ such that $x^{-1} V x \subseteq U$. By our hypothesis there exist $h \in H, z \in c_{G}(G)$, such that $x=h z$. Since $\tau$ is a group topology on $H$ there exist $V \in \mathcal{V}_{H, \tau}(1)$ such that $h^{-1} V h \subseteq U$. Then

$$
x^{-1} V x=z^{-1} h^{-1} V h z \subseteq z^{-1} U z=U
$$

as $z \in c_{G}(G)$. This proves that $\tau^{*}$ is a group topology of $G$.

Clearly, the condition $G=H c_{G}(G)$ is satisfied when $H$ is a central subgroup of $G$. It is satisfied also when $H$ is a direct summand of $G$. On the other hand, subgroups $H$ satisfying $G=H c_{G}(G)$ are normal.

Two questions are in order here:

- is the condition $G=H c_{G}(G)$ really necessary for the extension problems;

- is it possible to definite the extension $\tau^{*}$ in a different way in order to have always the possibility to extend a group topology?

\footnotetext{
${ }^{7}$ Hesse [66] showed that the use of $C H$ in Shelah's construction of a Markov group of size $\omega_{1}$ can be avoided.

${ }^{8}$ the Frattini subgroup of a group $G$ is the intersection of all maximal subgroups of $G$.
} 
Our next theorem shows that the difficulty of the extension problem are not hidden in the special features of the extension $\tau^{*}$.

Theorem 3.67. Let $H$ be a normal subgroup of the group $G$ and let $\tau$ be a group topology on $H$. Then the following are equivalent:

(a) the extension $\tau^{*}$ is a group topology on $G$;

(b) $\tau$ can be extended to a group topology of $G$;

(c) for every $x \in G$ the automorphism of $H$ induced by the conjugation by $x$ is $\tau$-continuous.

Proof. The implication $(\mathrm{a}) \rightarrow(\mathrm{b})$ is obvious, while the implication $(\mathrm{b}) \rightarrow(\mathrm{c})$ follows from the fact that the conjugations are continuous in any topological group. To prove the implication (c) $\rightarrow($ a) assume now that all automorphisms of $N$ induced by the conjugation by elements of $G$ are $\tau$-continuous. Take the filter of all neighborhoods of 1 in $\left(H, \tau^{*}\right)$ as a base of neighborhoods of 1 in the group topology $\tau^{*}$ of $G$. This works since the only axiom to check is to find for every $x \in G$ and every $\tau^{*}$-nbd $U$ of 1 a $\tau^{*}$-neighborhood $V$ of 1 such that $V^{x}:=x^{-1} V x \subseteq U$. Since we can choose $U, V$ contained in $H$, this immediately follows from our assumption of $\tau$-continuity of the restrictions to $H$ of the conjugations in $G$.

Now we give an example showing that the extension problem cannot be resolved for certain triples $G, H, \tau$ of a group $G$, its subgroup $H$ and a group topology $\tau$ on $H$.

Example 3.68. In order to produce an example when the extension is not possible we need to produce a triple $G, H, \tau$ such that at least some conjugation by an element of $G$ is not $\tau$-continuous when considered as an automorphism of $H$. The best tool to face this issue is the use of semi-direct products.

Let us recall that for groups $K, H$ and a group homomorphism $\theta: K \rightarrow A u t(H)$ one defines the semi-direct product $G=H \rtimes_{\theta} K$, where we shall identify $H$ with the subgroup $H \times\{1\}$ of $G$. In such a case, the conjugation in $G$ by an element $k$ of $K$ restricted to $H$ is precisely the automorphism $\theta(k)$ of $H$. Now consider a group topology $\tau$ on $H$. According to Theorem $3.67 \tau$ can be extended to a group topology of $G$ iff for every $k \in K$ the automorphism $\theta(k)$ of $H$ is $\tau$-continuous. (Indeed, every element $x \in G$ has the form $x=h k$, where $h \in H$ and $k \in K$; hence it remains to note that the conjugation by $x$ is composition of the (continuous) conjugation by $h$ and the conjugation by $k$.)

In order to produce the required example of a triple $G, H, \tau$ such that $\tau$ cannot be extended to $G$ it suffices to find a group $K$ and a group homomorphism $\theta: K \rightarrow A u t(H)$ such that at least one of the automorphisms $\theta(k)$ of $H$ is $\tau$-discontinuous. Of course, one can simplify the construction by taking the cyclic group $K_{1}=\langle k\rangle$ instead of the whole group $K$, where $k \in K$ is chosen such that the automorphisms $\theta(k)$ of $H$ is $\tau$-discontinuous. A further simplification can be arranged by taking $k$ in such a way that the automorphism $f=\theta(k)$ of $H$ is also an involition, i.e., $f^{2}=i d_{H}$. Then $H$ will be an index two subgroup of $G$.

Here is an example of a topological abelian group $(H, \tau)$ admitting a $\tau$-discontuous involition $f$. Then the triple $G, H, \tau$ such that $\tau$ cannot be extended to $G$ is obtained by simply taking $G=H \rtimes\langle f\rangle$, where the involition $f$ acts on $H$. Take as $(H, \tau)$ the torus group $\mathbb{T}$ with the usual topology. Then $\mathbb{T}$ is algebraically isomorphic to $(\mathbb{Q} / \mathbb{Z}) \oplus_{\mathfrak{c}} \bigoplus \mathbb{Q}$, so $\mathbb{T}$ has $2^{\mathfrak{c}}$ many involutions. Of these only the involutions $\pm i d_{\mathbb{T}}$ of $\mathbb{T}$ are continuous.

Let us conclude now with a series of examples when the extension problem has always a positive solution.

Example 3.69. Let $p$ be a prime number. If the group of $p$-adic integers $N=\mathbb{Z}_{p}$ is a normal subgroup of some group $G$, then the $p$-adic topology of $N$ can be extended to a group topology on $G$. Indeed, it suffices to note that if $\xi: N \rightarrow N$ is an automorphism of $N$, then $\xi\left(p^{n} N\right)=p^{n} N$. Since the subgroups $p^{n} N$ define the topology of $N$, this proves that every automorphism of $N$ is continuous. Now Theorem 3.67 applies.

Clearly, the $p$-adic integers can be replaced by any topological group $N$ such that every automorphism of $N$ is continuous (e.g., products of the form $\prod_{p} \mathbb{Z}_{p}^{k_{p}} \times F_{p}$, where $k_{p}<\omega$ and $F_{p}$ is a finite abelian $p$-group).

\subsection{Cardinal invariants of topological groups}

Here we shall be interested in measuring the minimum size of a base (of neighborhoods of 1) in a topological group $H$, as well as other cardinal functions related to $H$.

It is important to relate the bases (of neighborhoods of 1) in $H$ to those of a subgroup $G$ of $H$.

Exercise 3.70. If $G$ is a subgroup of a topological group $H$ and if $\mathcal{B}$ is a base (of neighborhoods of 1 ) in $H$ then a base (of neighborhoods of 1 ) in $G$ is given by $\{U \cap G: U \in \mathcal{B}\}$. 
Now we consider the case when $G$ is a dense subgroup of $H$.

Lemma 3.71. If $G$ is a dense subgroup of a topological group $H$ and $\mathcal{B}$ is a base of neighborhoods of 1 in $G$, then $\left\{\bar{U}^{H}: U \in \mathcal{B}\right\}$ is a base of neighborhoods of 1 in $H$.

Proof. Since the topological group $H$ is regular, the closed neighborhoods form a base at 1 in $H$. Hence for a neighborhood $V \ni 1$ in $H$ one can find another neighborhood $V_{0} \ni 1$ such that $\overline{V_{0}} \subseteq V$. Since $G \cap V_{0}$ is a neighborhood of 1 in $G$, there exists $U \in \mathcal{B}$ such that $U \subseteq G \cap V_{0}$. There exists also an open neighborhood $W$ of 1 in $H$ such that $U=W \cap G$. Obviously, one can choose $W \subseteq V_{0}$. Hence $\bar{U}^{H}=\bar{W}$ as $G$ is dense in $H$ and $W$ is open in $H$. Thus $\bar{U}^{H}=\bar{W} \subseteq \bar{V}_{0} \subseteq V$ is a neighborhood of 1 in $H$.

Lemma 3.72. Let $G$ be a dense subgroup of a topological group $H$ and let $\mathcal{B}$ be a base of neighborhoods of 1 in $H$. Then $\{g U: U \in \mathcal{B}, g \in G\}$ is a base of the topology of $H$.

Proof. Let $x \in H$ and let $x \in O$ be an open set. Then there exists a $U \in \mathcal{U}$ symmetric with $x U^{2} \subseteq O$. Pick a $g \in G \cap x U$. Then $x \in g U \subseteq O$.

For a topological group $G \operatorname{set} d(G)=\min \{|X|: X$ is dense in $G\}$,

$$
w(G)=\min \{|\mathcal{B}|: \mathcal{B} \text { is a base of } G\} \text { and } \chi(G)=\min \{|\mathcal{B}|: \mathcal{B} \text { a base of neighborhoods of } 1 \text { in } G\} .
$$

Lemma 3.73. Let $H$ be a subgroup of a topological group $G$. Then:

(a) $w(H) \leq w(G)$ and $\chi(H) \leq \chi(G)$;

(b) if $H$ is dense in $G$, then $w(G)=w(H)$ and $\chi(G)=\chi(H)$.

Lemma 3.74. $w(G)=\chi(G) \cdot d(G)$ for every topological group $G$.

Proof. The inequality $w(G) \geq \chi(G)$ is obvious. To see that $w(G) \geq d(G)$ choose a base $\mathcal{B}$ of size $w(G)$ and for every $U \in \mathcal{B}$ pick a point $d_{U} \in U$. Then the set $D=\left\{d_{U}: U \in \mathcal{B}\right\}$ is dense in $G$ and $|D| \leq w(G)$. This proves the inequality $w(G) \geq \chi(G) \cdot d(G)$.

The inequality $w(G) \leq \chi(G) \cdot d(G)$ follows from the previous lemma.

The cardinal invariants of the topological groups are cardinal numbers, say $\rho(G)$, associated to every topological group $G$ such that if $G$ is topologically isomorphic to the topological group $H$, then $\rho(G)=\rho(H)$. For example, the size $|G|$ is the simplest cardinal invariant of a topological group, it does not depend on the topology of $G$. Other cardinal invariants are the weight $w(G)$, the character $\chi(G)$ and the density character $d(G)$ defined above. Beyond the equality $w(G)=\chi(G) \cdot d(G)$ proved in Lemma 3.74, one has also the following inequalities:

Lemma 3.75. Let $G$ be a topological group. Then:

(a) $d(G) \leq w(G) \leq 2^{d(G)}$;

(b) $|G| \leq 2^{w(G)}$ if $G$ is Hausdorff.

Proof. (a) $d(G) \leq w(G)$ has already been proved in Lemma 3.74 (a). To prove $w(G) \leq 2^{d(G)}$ note that $G$ is regular, hence every open base $\mathcal{B}$ on $G$ contains a base $\mathcal{B}_{r}$ of the same size consisting of regular open sets ${ }^{9}$. Let $\mathcal{B}$ be a base of $G$ of regular open sets and let $D$ be a dense subgroup of $G$ of size $d(G)$. If $U, V \in \mathcal{B}$, with $U \cap D=V \cap D$, then $\bar{U}=\overline{U \cap D}=\overline{V \cap D}=\bar{V}$. Being $U$ and $V$ regular open, the equality $\bar{U}=\bar{V}$ implies $U=V$. Hence the map $U \mapsto U \cap D$ from $\mathcal{B}$ to the power set $P(D)$ is injective. Therefore $w(G) \leq 2^{d(G)}$.

(b) To every point $x \in G$ assign the set $O_{x}=\{U \in \mathcal{B}: x \in U\}$. Then the axiom $T_{2}$ guarantees that map $x \mapsto O_{x}$ from $G$ to the power set $P(\mathcal{B})$ is injective. Therefore, $|G| \leq 2^{w(G)}$.

Remark 3.76. Two observations related to item (b) of the above lemma are in order here.

- The equality in item (b) can be attained (see Theorem 4.46).

- One cannot remove Hausdorffness in item (b) (any large indiscrete group provides a counter-example). This dependence on separation axioms is due to that the presence of the size of the group in (b). We see in the next exercise that the Hausdorff axiom is not relevant as far as the other cardinal invariants are involved.

\footnotetext{
${ }^{9}$ an open set is said to be regular open if it coincides with the interior of its closure.
} 
Exercise 3.77. Let $G$ be a topological group. Prove that:

- $w(G)=w(G / \overline{\{1\}}), \chi(G)=\chi(G / \overline{\{1\}})$ and $d(G)=d(G / \overline{\{1\}})$;

- $d(U)=D(G)$ for every non-empty open set $U$, if $G$ is Lindelöff;

- $w(G)=\chi(G)$ if locally compact and $\sigma$-compact.

\subsection{Completeness and completion}

A net $\left\{g_{\alpha}\right\}_{\alpha \in A}$ in a topological group $G$ is a Cauchy net if for every neighborhood $U$ of 1 in $G$ there exists $\alpha_{0} \in A$ such that $g_{\alpha}^{-1} g_{\beta} \in U$ and $g_{\beta} g_{\alpha}^{-1} \in U$ for every $\alpha, \beta>\alpha_{0}$.

Exercise 3.78. Let $G$ be a dense subgroup of a topological group $H$. If $\left(g_{\alpha}\right)$ is a net in $G$ that converges to some element $h \in H$, then $\left(g_{\alpha}\right)$ is a Cauchy net.

By the previous exercise, the convergent nets are Cauchy nets. A topological group $G$ is complete (in the sense of Raikov) if every Cauchy net in $G$ converges in $G$. We omit the tedious proof of the next theorem.

Theorem 3.79. For every topological Hausdorff group $G$ there exists a complete topological group $\widetilde{G}$ and a topological embedding $i: G \rightarrow \widetilde{G}$ such that $i(G)$ is dense in $\widetilde{G}$. Moreover, if $f: G \rightarrow H$ is a continuous homomorphism and $H$ is a complete topological group, then there is a unique continuous homomorphism $\widetilde{f}$ : $\widetilde{G} \rightarrow H$ with $f=\widetilde{f} \circ i$.

Therefore every Hausdorff topological abelian group has a unique, up to topological isomorphisms, (Raikovcompletion $(\widetilde{G}, i)$ and we can assume that $G$ is a dense subgroup of $\widetilde{G}$.

Definition 3.80. A net $\left\{g_{\alpha}\right\}_{\alpha \in A}$ in $G$ is a left [resp., right] Cauchy net if for every neighborhood $U$ of 1 in $G$ there exists $\alpha_{0} \in A$ such that $g_{\alpha}^{-1} g_{\beta} \in U$ [resp., $g_{\beta} g_{\alpha}^{-1} \in U$ ] for every $\alpha, \beta>\alpha_{0}$.

Lemma 3.81. Let $G$ be a Hausdorff topological group. Every left (resp., right) Cauchy net in $G$ with a convergent subnet is convergent.

Proof. Let $\left\{g_{\alpha}\right\}_{\alpha \in A}$ be a left Cauchy net in $G$ and let $\left\{g_{\beta}\right\}_{\beta \in B}$ be a subnet convergent to $x \in G$, where $B$ is a cofinal subset of $A$. Let $U$ be a neighborhood of 1 in $G$ and $V$ a symmetric neighborhood of 1 in $G$ such that $V V \subseteq U$. Since $g_{\beta} \rightarrow x$, there exists $\beta_{0} \in B$ such that $g_{\beta} \in x V$ for every $\beta>\beta_{0}$. On the other hand, there exists $\alpha_{0} \in A$ such that $\alpha_{0} \geq \beta_{0}$ and $g_{\alpha}^{-1} g_{\gamma} \in V$ for every $\alpha, \gamma>\alpha_{0}$. With $\gamma=\beta_{0}$ we have $g_{\alpha} \in x V V \subseteq x U$ for every $\alpha>\alpha_{0}$, that is $g_{\alpha} \rightarrow x$.

A topological group $G$ is complete in the sense of Weil if every left Cauchy net converges in $G$.

Every Weil-complete group is also complete, but the converse does not hold in general. It is possible to define the Weil-completion of a Hausdorff topological group in analogy with the Ralkov-completion.

Exercise 3.82. Prove that if a Hausdorff topological group $G$ admits a Weil-completion, then in $G$ the left Cauchy and the right Cauchy nets coincide.

Exercise 3.83. Let $X$ be an infinite set and let $G=S(X)$ equipped with the topology described in $\S 3.2 .4$. Prove that:

(a) a net $\left\{f_{\alpha}\right\}_{\alpha \in A}$ in $G$ is left Cauchy iff there exists $f \in X^{X}$ so that $f_{\alpha} \rightarrow f$ in $X^{X}$, prove that such an $f$ must necessarily be injective;

(b) a net $\left\{f_{\alpha}\right\}_{\alpha \in A}$ in $G$ is right Cauchy iff there exists $g \in X^{X}$ so that $f_{\alpha}^{-1} \rightarrow g$ in $X^{X}$;

(c) the group $S(X)$ admits no Weil-completion. (Hint. Build a left Cauchy net in $S(X)$ that is not right Cauchy and use items (a) and (b), as well as the previous exercise.)

(d) $S(X)$ is Raikov-complete. (Hint. Use items (a) and (b).)

Exercise 3.84. (a) Let $G$ be a linearly topologized group and let $\left\{N_{i}: i \in I\right\}$ be its system of neighborhoods of 1 consisting of open normal subgroups. Then the completion of $G$ is isomorphic to the inverse limit $\lim _{\leftarrow} G / N_{i}$ of the discrete quotients $G / N_{i}$.

(b) Show that the completion in (a) is compact iff all $N_{i}$ have finite index in $G$. 
(c) Let $p$ be a prime number. Prove that the completion of $\mathbb{Z}$ equipped with the p-adic topology (see Example 3.7) is the compact group $\mathbb{J}_{p}$ of p-adic integers.

(d) Prove that the completion of $\mathbb{Z}$ equipped with the natural topology (see Example 3.7) is isomorphic to $\prod_{p} \mathbb{J}_{p}$.

Exercise 3.85. Let $p$ be a prime number. Prove that:

(a) $\mathbb{Z}$ admits a finest group topology $\tau$ such that $p^{n}$ converges to 0 in $\tau$ (this is $\tau_{\left(p^{n}\right)}$ in the notation of $\left.\S 3.4\right)$;

(b)* [89, 88$](\mathbb{Z}, \tau)$ is complete;

(c) conclude that $\tau$ is not metrizable.

Exercise 3.86. Let $G$ be a Hausdroff topologized group. Call a filter $\mathcal{F}$ on $G$ Cauchy if for every $U \in \mathcal{V}_{G}(1)$ there exists $g \in G$ such that $g U \in \mathcal{F}$. Prove that:

(a) a filter $\mathcal{F}$ on $G$ Cauchy iff for every $U \in \mathcal{V}_{G}(1)$ there exists $g \in G$ such that $U g \in \mathcal{F}$.

(b) if $\mathcal{F}$ is a Cauchy filter on $G$ and $x_{F} \in F$ for every $F \in \mathcal{F}$, then the net $\left\{x_{F}: F \in \mathcal{F}\right\}$ is a Cauchy net (here $\mathcal{F}$ is considered as a directed partially ordered set w.r.t. inclusion);

(c) if $\left\{x_{i}: i \in I\right\}$ is a Cauchy net in $G$ and $F_{i}=\left\{x_{j}: j \in I, j \geq i\right\}$, then the family $\left\{F_{i}: i \in I\right\}$ is a filter base of a Cauchy filter on $G$;

(d) $G$ is complete iff every Cauchy filter in $G$ converges.

\section{Compactness and local compactness in topological groups}

Clearly, a topological group $G$ is locally compact if there exists a compact neighborhood of $e_{G}$ in $G$ (compare with Definition 2.10). We shall assume without explicitly mentioning it, that all locally compact groups are Hausdorff.

As an immediate consequence of Tychonov's theorem of compactness of products we obtain the following the first example of a compact abelian group (it will become clear with the duality theorem that this is the most general one).

Remark 4.1. Let us see that for every abelian group $G$ the group $G^{*}=\operatorname{Hom}(G, \mathbb{S})$ is closed in the product $\mathbb{S}^{G}$, hence $G^{*}$ is compact. Consider the projections $\pi_{x}: \mathbb{S}^{G} \rightarrow \mathbb{T}$ for every $x \in G$ and the following equalities

$$
\begin{aligned}
G^{*} & =\bigcap_{h, g \in G}\left\{f \in \mathbb{S}^{G}: f(h+g)=f(h) f(g)\right\}=\bigcap_{h, g \in G}\left\{f \in \mathbb{S}^{G}: \pi_{h+g}(f)=\pi_{h}(f) \pi_{g}(f)\right\} \\
& =\bigcap_{h, g \in G}\left\{f \in \mathbb{S}^{G}:\left(\pi_{h+g}^{-1} \pi_{h} \pi_{g}\right)(f)=1\right\}=\bigcap_{h, g \in G} \operatorname{ker}\left(\pi_{h+g}^{-1} \pi_{h} \pi_{g}\right) .
\end{aligned}
$$

Since $\pi_{x}$ is continuous for every $x \in G$ and $\{1\}$ is closed in $\mathbb{S}$, then all $\operatorname{ker}\left(\pi_{h+g}^{-1} \pi_{h} \pi_{g}\right)$ are closed; so $\operatorname{Hom}(G, \mathbb{S})$ is closed too.

The next lemma contains a well known useful fact - the existence of a "diagonal subnet".

Lemma 4.2. Let $G$ be an abelian group and let $N=\left\{\chi_{\alpha}\right\}_{\alpha}$ be a net in $G^{*}$. Then there exist $\chi \in G^{*}$ and a subnet $S=\left\{\chi_{\alpha_{\beta}}\right\}_{\beta}$ of $N$ such that $\chi_{\alpha_{\beta}}(x) \rightarrow \chi(x)$ for every $x \in G$.

Proof. By Tychonov's theorem, the group $\mathbb{S}^{G}$ endowed with the product topology is compact. Then $N$ has a convergent (to $\chi$ ) subnet $S$. Therefore $\chi_{\alpha_{\beta}}(x) \rightarrow \chi(x)$ for every $x \in G$ and $\chi \in G^{*}$, because $G^{*}$ is closed in $\mathbb{S}^{G}$ by 4.1 .

\subsection{Specific properties of (local) compactness}

Here we shall see the impact of local compactness in various directions (the open mapping theorem, properties related to connectedness, etc.).

Lemma 4.3. Let $G$ be a topological group and let $C$ and $K$ be closed subsets of $G$ : 
(a) if $K$ is compact, then both $C K$ and $K C$ are closed;

(b) if both $C$ and $K$ are compact, then $C K$ and $K C$ are compact;

(c) if $K$ is contained in an open subset $U$ of $G$, then there exists an open neighborhood $V$ of 1 such that $K V \subseteq U$

Proof. (a) Let $\left\{x_{\alpha}\right\}_{\alpha \in A}$ be a net in $C K$ such that $x_{\alpha} \rightarrow x_{0} \in G$. It is sufficient to show that $x_{0} \in C K$. For every $\alpha \in A$ we have $x_{\alpha}=y_{\alpha} z_{\alpha}$, where $y_{\alpha} \in C$ and $z_{\alpha} \in K$. Since $K$ is compact, then there exist $z_{0} \in K$ and a subnet $\left\{z_{\alpha_{\beta}}\right\}_{\beta \in B}$ such that $z_{\alpha_{\beta}} \rightarrow z_{0}$. Thus $\left(x_{\alpha_{\beta}}, z_{\alpha_{\beta}}\right)_{\beta \in B}$ is a net in $G \times G$ which converges to $\left(x_{0}, z_{0}\right)$. Therefore $y_{\alpha_{\beta}}=x_{\alpha_{\beta}} z_{\alpha_{\beta}}^{-1}$ converges to $x_{0} z_{0}^{-1}$ because the function $(x, y) \mapsto x y^{-1}$ is continuous. Since $y_{\alpha_{\beta}} \in C$ for every $\beta \in B$ and $C$ is closed, $x_{0} z_{0}^{-1} \in C$. Now $x_{0}=\left(x_{0} z_{0}^{-1}\right) z_{0} \in C K$. Analogously it is possible to prove that $K C$ is closed.

(b) The product $C \times K$ is compact by the Tychonov theorem and the function $(x, y) \mapsto x y$ is continuous and maps $C \times K$ onto $C K$. Thus $C K$ is compact.

(c) Let $C=G \backslash U$. Then $C$ is a closed subset of $G$ disjoint with $K$. Therefore, for the compact subset $K^{-1}$ of $G$ one has $1 \notin K^{-1} C$. By (a) $K^{-1} C$ is closed, so there exists a symmetric neighborhood $V$ of 1 that misses $K^{-1} C$. Then $K V$ misses $C$ and consequently $K V$ is contained in $U$.

Compactness of $K$ cannot be omitted in item (a). Indeed, $K=\mathbb{Z}$ and $C=\langle\sqrt{2}\rangle$ are closed subgroups of $G=\mathbb{R}$ but the subgroup $K+C$ of $\mathbb{R}$ is dense (see Exercie 3.20 or Proposition 4.45 ).

Lemma 4.4. Let $G$ be a topological group and $K$ a compact subgroup of $G$. Then the canonical projection $\pi: G \rightarrow G / K$ is closed.

Proof. Let $C$ be a closed subset of $G$. Then $C K$ is closed by Lemma 4.3 and so $U=G \backslash C K$ is open. For every $x \notin C K$, that is $\pi(x) \notin \pi(C), \pi(U)$ is an open neighborhood of $\pi(x)$ such that $\pi(U) \cap \pi(C)$ is empty. So $\pi(C)$ is closed.

Lemma 4.5. Let $G$ be a topological group and let $H$ be a closed subgroup of $G$.

(1) If $G$ is compact, then $G / H$ is compact.

(2) If $H$ and $G / H$ are compact, then $G$ is compact.

Proof. (1) is obvious.

(2) Let $\mathcal{F}=\left\{F_{\alpha}: \alpha \in A\right\}$ be a family of closed sets of $G$ with the finite intersection property. If $\pi: G \rightarrow G / H$ is the canonical projection, $\pi(\mathcal{F})$ is a family of closed subsets with the finite intersection property in $G / H$ by Lemma 4.4. By the compactness of $G / H$ there exists $\pi(x) \in \pi\left(F_{\alpha}\right)$ for every $\alpha \in A$. So $x \in \bigcap_{\alpha \in A} F_{\alpha} H$. Let $x=f_{\alpha} h_{\alpha}$ with $h_{\alpha} \in H$ and $f_{\alpha} \in F_{\alpha}$. It is not restrictive to assume that $\mathcal{F}$ is closed for finite intersections. Define a partial order on $A$ by $\alpha \leq \alpha^{\prime}$ if $F_{\alpha} \supseteq F_{\alpha^{\prime}}$. Then $(A, \leq)$ is a right-filtered partially ordered set and so $\left\{f_{\alpha}\right\}_{\alpha \in A}$ is a net in $G$. By the compactness of $H$ we can assume wlog that $h_{\alpha}$ converges to $h \in H$ (otherwise pass to a convergent subnet). But then $f_{\alpha}=x h_{\alpha}^{-1} \rightarrow x h^{-1}$. Since $f_{\alpha}$ is contained definitively in $F_{\alpha}$, also the limit $x h^{-1} \in F_{\alpha}$. So the intersection of all $F_{\alpha}$ is not empty.

Lemma 4.6. Let $G$ be a locally compact group, $H$ be a closed subgroup of $G$ and $\pi: G \rightarrow G / H$ be the canonical projection. Then:

(a) $\mathrm{G} / \mathrm{H}$ is locally compact too;

(b) If $C$ is a compact subset of $G / H$, then there exists a compact subset $K$ of $G$ such that $\pi(K)=C$.

Proof. Let $U$ be an open neighborhood of 1 in $G$ with compact closure. Consider the open neighborhood $\pi(U)$ of 1 in $G / H$. Then $\pi(\bar{U}) \subseteq \overline{\pi(U)}$ by the continuity of $\pi$. Now $\pi(\bar{U})$ is compact in $G / H$, which is Hausdorff, and so $\pi(\bar{U})$ is closed. Since $\pi(U)$ is dense in $\pi(\bar{U})$, we have $\overline{\pi(U)}=\overline{\pi(\bar{U})}=\pi(\bar{U})$. So $G / H$ is locally compact.

(b) Let $U$ be an open neighborhood of 1 in $G$ with compact closure. Then $\{\pi(s U): s \in G\}$ is an open covering of $G / H$. Since $C$ is compact, a finite subfamily $\left\{\pi\left(s_{i} U\right): i=1, \ldots, m\right\}$ covers $C$. Then we can take $K=\left(s_{1} U \cup \cdots \cup s_{m} U\right) \cap \pi^{-1}(C)$.

Lemma 4.7. A locally compact group is Weil-complete. 
Proof. Let $U$ be a neighborhood of 1 in $G$ with compact closure and let $\left\{g_{\alpha}\right\}_{\alpha \in A}$ be a left Cauchy net in $G$. Then there exists $\alpha_{0} \in A$ such that $g_{\alpha}^{-1} g_{\beta} \in U$ for every $\alpha, \beta \geq \alpha_{0}$. In particular, $g_{\beta} \in g_{\alpha_{0}} U$ for every $\beta>\alpha_{0}$. By the compactness of $g_{\alpha_{0}} \bar{U}$, we can conclude that there exists a convergent subnet $\left\{g_{\beta}\right\}_{\beta \in B}$ (for some cofinal $B \subseteq A$ ) such that $g_{\beta} \rightarrow g \in G$. Then also $g_{\alpha}$ converges to $g$ by Lemma 3.81 .

Lemma 4.8. A locally compact countable group is discrete.

Proof. By the Baire category theorem $2.16 G$ is of second category. Since $G=\left\{g_{1}, \ldots, g_{n}, \ldots\right\}=\bigcup_{n=1}^{\infty}\left\{g_{n}\right\}$, there exists $n \in \mathbb{N}_{+}$such that Int $\left\{g_{n}\right\}$ is not empty and so $\left\{g_{n}\right\}$ is open.

Now we prove the open mapping theorem for topological groups.

Theorem 4.9 (Open mapping theorem). Let $G$ and $H$ be locally compact topological groups and let $f$ be a continuous homomorphism of $G$ onto $H$. If $G$ is $\sigma$-compact, then $f$ is open.

Proof. Let $U$ be an open neighborhood of 1 in $G$. There exists an open symmetric neighborhood $V$ of 1 in $G$ such that $\overline{V V} \subseteq U$ and $\bar{V}$ is compact. Since $G=\bigcup_{x \in G} x V$ and $G$ is Lindelöff by Lemma 2.17, we have $G=\bigcup_{n=1}^{\infty} x_{n} V$. Therefore $H=\bigcup_{n=1}^{\infty} h\left(x_{n} \bar{V}\right)$, because $h$ is surjective. Put $y_{n}=h\left(x_{n}\right)$, hence $H=\bigcup_{n=1}^{\infty} y_{n} h(\bar{V})$ where each $h(\bar{V})$ is compact and so closed in $H$. Since $H$ is locally compact, Theorem 2.16 yields that there exists $n \in \mathbb{N}_{+}$such that Int $h(\bar{V})$ is not empty. So there exists a non-empty open subset $W$ of $H$ such that $W \subseteq h(\bar{V})$. If $w \in W$, then $w \in h(\bar{V})$ and so $w=h(v)$ for some $v \in \bar{V}=\bar{V}^{-1}$. Hence

$$
1 \in w^{-1} W \subseteq w^{-1} h(\bar{V})=h\left(v^{-1}\right) h(\bar{V}) \subseteq h(\overline{V V}) \subseteq h(U)
$$

and this implies that $h(U)$ is an open neighborhood of 1 in $H$.

The following immediate corollary is frequently used:

Corollary 4.10. If $f: G \rightarrow H$ is a continuous surjective homomorphism of Hausdorff topological groups and $G$ is compact, then $f$ is open.

Now we introduce a special class of $\sigma$-compact groups that will play an essential role in determining the structure of the locally compact abelian groups.

Definition 4.11. A group $G$ is compactly generated if there exists a compact subset $K$ of $G$ which generates $G$, that is $G=\langle K\rangle=\bigcup_{n=1}^{\infty}\left(K \cup K^{-1}\right)^{n}$.

Lemma 4.12. If $G$ is a compactly generated group then $G$ is $\sigma$-compact.

Proof. By the definition $G=\bigcup_{n=1}^{\infty}\left(K \cup K^{-1}\right)^{n}$, where every $\left(K \cup K^{-1}\right)^{n}$ is compact, since $K$ is compact.

It should be emphasized that while $\sigma$-compactness is a purely topological property, being compactly generated involves essentially the algebraic structure of the group.

Exercise 4.13. (a) Give examples of $\sigma$-compact groups that are not compactly generated.

(b) Show that every connected locally compact group is compactly generated.

Lemma 4.14. Let $G$ be a locally compact group.

(a) If $K$ a compact subset of $G$ and $U$ an open subset of $G$ such that $K \subseteq U$, then there exists an open neighborhood $V$ of 1 in $G$ such that $(K V) \cup(V K) \subseteq U$ and $\overline{(K V) \cup(V K)}$ is compact.

(b) If $G$ is compactly generated, then there exists an open neighborhood $U$ of 1 in $G$ such that $\bar{U}$ is compact and $U$ generates $G$.

Proof. (a) By Lemma 4.3 (c) there exists an open neighborhood $V$ of 1 in $G$ such that $(K V) \cup(V K) \subseteq U$. Since $G$ is locally compact, we can choose $V$ with compact closure. Thus $K \bar{V}$ is compact by Lemma 4.3 . Since $K V \subseteq K \bar{V}$, then $\overline{K V} \subseteq K \bar{V}$ and so $\overline{K V}$ is compact. Analogously $\overline{V K}$ is compact, so $\overline{(K V) \cup(V K)}=\overline{K V} \cup \overline{V K}$ is compact.

(b) Let $K$ be a compact subset of $G$ such that $K$ generates $G$. So $K \cup\{1\}$ is compact and by (a) there exists an open neighborhood $U$ of 1 in $G$ such that $U \supseteq K \cup\{1\}$ and $\bar{U}$ is compact.

In the case of first countable topological groups Fujita and Shakmatov [49] have described the precise relationship between $\sigma$-compactness and the property of being compactly generated. 
Theorem 4.15. A metrizable topological group $G$ is compactly generated if and only if $G$ is $\sigma$-compact and, for every open subgroup $H$ of $G$, there exists a finite set $F \subseteq G$ such that $F \cup H$ algebraically generates $G$ [49].

This gives the following:

Corollary 4.16. A $\sigma$-compact metrizable group $G$ is compactly generated in each of the following cases (for the definition of total boundedness see Definition 4.25):

- $G$ has no open subgroups

- the completion $\widetilde{G}$ is connected;

- $G$ is totally bounded.

Moreover,

Theorem 4.17. A countable metrizable group is compactly generated iff it is algebraically generated by a sequence (possibly eventually constant) converging to its neutral element.

Examples showing that the various conditions above cannot be omitted can be found in [49]. $[50]$.

The question when will a topological group contain a compactly generated dense subgroup is considered in

Now we see that linearity and total disconnectedness of group topologies coincide for compact groups and for locally compact abelian groups.

Theorem 4.18. Every locally compact totally disconnected group has a base of neighborhoods of 1 consisting of open subgroups. In particular, a locally compact totally disconnected group that is either abelian or compact has linear topology.

This can be derived from the followis more precise result:

Theorem 4.19. Let $G$ be a locally compact topological group and let $C=c(G)$. Then :

(a) $C$ coincides with the intersection of all open subgroups of $G$;

(b) if $G$ is totally disconnected, then every neighborhoodof 1 contains an open subgroup of $G$.

If $G$ is compact, then the open subgroups in items (a) and (b) can be chosen normal.

Proof. (a) follows from (b) as $G / C$ is totally disconnected hence the neutral element of $G / C$ is intersection of open (resp. open normal) subgroups of $G / C$. Now the intersection of the inverse images, w.r.t. the canonical homomorphism $G \rightarrow G / C$, of these subgroups coincides with $C$.

(b) Let $G$ be a locally compact totally disconnted group. By Vedenissov's Theorem $G$ has a base $\mathcal{O}$ of clopen symmetric compact neighborhoods of 1 . Let $U \in \mathcal{O}$. The $U=\bar{U}=\bigcap_{V \in \mathcal{O}} U V$. Then every set $U \cdot V$ is compact by Lemma 4.3, hence closed. According to Lemma 2.14 there exist $V_{1}, \ldots, V_{n} \in \mathcal{O}$ such that $U=\bigcap_{k=1}^{n} U V_{k}$. Then for $V:=U \cap \bigcap_{k=1}^{n} V_{k}$ one has $U V=U$. This implies also $V V \subseteq U, V V V \subseteq U$ etc. Since $V$ is symmetric, the subgroup $H=\langle V\rangle$ is contained in $U$ as well. From $V \subseteq H$ one can deduce that $H$ is open (cf. 3.16). In case $G$ is compact, note that the heart $H_{G}=\bigcap_{x \in G} x^{-1} H x$ of $H$ is an open normal subgroup as the number of all conjugates $x^{-1} H x$ of $H$ is finite (being equal to $\left[G: N_{G}(H)\right] \leq[G: H]<\infty$ ). Hence $H_{G}$ is an open normal subgroup of $G$ contained in $H$, hence also in $U$.

Corollary 4.20. The quotient of a locally compact totally disconnected group is totally disconnected.

Proof. Let $G$ be a locally compact totally disconnected group and let $N$ be a closed normal subgroup of $G$. It follows from the above theorem that $G$ has a linear topology. This yields that the quotient $G / N$ has a linear topology too. Thus $G / N$ is totally disconnected.

Corollary 4.21. The continuous homomorphic images of compact totally disconnected groups are totally disconnected.

Proof. Follows from the above corollary and the open mapping theorem.

According to Example 3.46 none of the items (a) and (b) of the theorem remain true without the hypothesis "locally compact".

Corollary 4.22. Let $G$ be a locally compact group. Then $Q(G)=c(G)$.

Proof. By item (a) of the above theorema $C(G)$ is an intersection of open subgroups, that are clopen being open subgroups (cf. Proposizione 3.16). Hence $c(G)$ contains $Q(G)$ which in turn coincides with the intersection of all clopen sets of $G$ containing 1 . The inclusion $C(G) \subseteq Q(G)$ is always true. 


\subsection{Subgroups of the compact groups}

For a subset $E$ of an abelian group $G$ we set $E_{(2)}=E-E, E_{(4)}=E-E+E-E, E_{(6)}=E-E+E-E+E-E$ and so on.

A subset $X$ of an abelian group $(G,+)$ is $b i g^{10}$ if there exists a finite subset $F$ of $G$ such that $G=X+F$. Obviously, every non-empty set of a finite group is big; on the other hand, every big set in an infinite group is necessarily infinite.

Exercise 4.23. Let $B$ be an infinite subset of $\mathbb{Z}$. Show that $B$ is big iff the following two conditions hold:

(a) $B$ is unbounded from above and from below;

(b) if $B=\left\{b_{n}\right\}_{n=-\infty}^{\infty}$ is a one-to-one monotone enumeration of $B$ then the differences $b_{n+1}-b_{n}$ are bounded.

Exercise 4.24. (a) Assume $B_{\nu}$ is a big set of the group $G_{\nu}$ for $\nu=1,2, \ldots, n$. Prove that $B_{1} \times \ldots \times B_{n}$ is a big set of $G_{1} \times \ldots \times G_{n}$.

(b) if $f: G \rightarrow H$ is a surjective group homomorphism and $B$ is a big subset of $H$, then $f^{-1}(B)$ is a big subset of $G$.

Definition 4.25. A topological group $G$ is totally bounded if every open non-empty subset $U$ of $G$ is big. A Hausdorff totally bounded group will be called precompact.

Clearly, compact groups are precompact.

Note that if $f$ in item (b) of 4.24 is not surjective, then the property may fail. The next proposition gives an easy remedy to this.

Proposition 4.26. Let $A$ be an abelian group and let $B$ be a big subset of $A$. Then $(B-B) \cap H$ is big with respect to $H$ for every subgroup $H$ of $A$.

If $a \in A$ then there exists a sufficiently large positive integer $n$ such that na $\in B-B$.

Proof. There exists a finite subset $F$ of $A$ such that $B+F=A$. For every $f \in F$, if $(B+f) \cap H$ is not empty, choose $a_{f} \in(B+f) \cap H$, and if $(B+f) \cap H$ is empty, choose an arbitrary $a_{f} \in H$. On the other hand, for every $x \in H$ there exists $f \in F$ such that $x \in B+f$; since $a_{f} \in B+f$, we have $x-a_{f} \in B-B$ and so $H \subseteq(B-B) \cap H+\left\{a_{f}: f \in F\right\}$, that is $(B-B) \cap H$ is big in $H$.

For the last assertion it suffices to take $H=\langle a\rangle$. If $H$ is finite, then there is nothing to prove as $0 \in B-B$. Otherwise $H \cong \mathbb{Z}$ so the first item of Exercise 4.23 applies.

Combining this proposition with item (b) of 4.24 we get:

Corollary 4.27. For every group homomorphism $f: G \rightarrow H$ and every big subset $B$ of $H$, the subset $f^{-1}(B-B)$ of $G$ is a big.

Here comes the most important consequence of the above proposition.

Corollary 4.28. Subgroups of precompact groups are precompact. In particular, all subgroups of compact groups are precompact.

One can show that the precompact groups are precisely the subgroups of the compact groups. This requires two steps as the next theorem shows:

Theorem 4.29. (a) A group having a dense precompact subgroup is necessarily precompact.

(b) The compact groups are precisely the complete precompact groups.

Proof. (a) Indeed, assume that $H$ is a dense precompact subgroup of a group $G$. Then for every $U \in \mathcal{V}_{G}(0)$ choose an open $V \in \mathcal{V}_{G}(0)$ with $V+V \subseteq U$. By the precompactness of $H$ there exists a finite set $F \subseteq H$ such that $H=F+V \cap H$. Then

$$
G=V+H \subseteq V+F+V \cap H \subseteq F+V+V \subseteq F+U .
$$

\footnotetext{
${ }^{10}$ Some authors use also the terminology large, relatively dense, or syndetically dense. This notion can be given for non-abelian groups as well, but then both versions, left large and right large, do not coincide. This creates some technical difficulties that we prefer to avoid since the second part of this section is relevant only for abelian groups. The first half, including the characterization 4.29 , remains valid in the non-abelian case as well (since, fortunately, the "left" and "right" versions of total boundedness coincide).
} 
(b) Compact groups are complete and precompact. To prove the other implication take a complete precompact group $G$. To prove that $G$ is compact it sufficies to prove that every ultrafilre on $G$ converges. Assume $\mathcal{U}$ is such an ultrafiler. We show first that it is a Cauchy filter. Indeed, if $U \in \mathcal{V}_{G}(0)$, then $U$ is a big set of $G$ so there exists $g_{1}, g_{2}, \ldots, g_{n} \in G$ such that $G=\bigcup_{i=1}^{n} g_{i}+U$. Since $\mathcal{U}$ is an ultrafilter, $g_{i}+U \in \mathcal{U}$ for some $i$. Hence $\mathcal{U}$ is a is a Cauchy filter. According to Exercise $3.86 \mathcal{U}$ converges.

In this way we have described the precompact groups internally (as the Hausdorff topological groups having big non-empty open sets), or externally (as the subgroups of the compact groups).

Now we adopt a different approach to describe the precompact groups, based on the use of characters. Our first aim will be to see that the topologies induced by characters are always totally bounded.

Proposition 4.30. If $A$ is an abelian group, $\delta>0$ and $\chi_{1}, \ldots, \chi_{s} \in A^{*}\left(s \in \mathbb{N}_{+}\right)$, then $U\left(\chi_{1}, \ldots, \chi_{s} ; \delta\right)$ is big in A. Moreover for every $a \in A$ there exists a sufficiently large positive integer $n$ such that na $\in U\left(\chi_{1}, \ldots, \chi_{s} ; \delta\right)$.

Proof. Define $h: A \rightarrow \mathbb{T}^{s}$ such that $h(x)=\left(\chi_{1}(x), \ldots, \chi_{s}(x)\right)$ and

$$
B=\left\{\left(z_{1}, \ldots, z_{n}\right) \in \mathbb{S}^{s}:\left|\operatorname{Arg} z_{i}\right|<\frac{\delta}{2} \text { for } i=1, \ldots, s\right\}=\left\{z \in \mathbb{S}:|\operatorname{Arg} z|<\frac{\delta}{2}\right\}^{s} .
$$

Then $B$ is big in $\mathbb{S}^{s}$ and by Proposition 4.26 the set $(B-B) \cap h(A)$ is big with respect to $h(A)$. Since

$$
B-B \subseteq C=\left\{\left(z_{1}, \ldots, z_{s}\right) \in \mathbb{S}^{s}:\left\|\operatorname{Arg} z_{i}\right\|<\delta \text { for } i=1, \ldots, s\right\}
$$

we have that $C \cap h(A)$ is big with respect to $h(A)$. Therefore $U\left(\chi_{1}, \ldots, \chi_{s} ; \delta\right)=h^{-1}(C)$ is big in $A$.

The second statement follows from Proposition 4.26, since

$$
U\left(\chi_{1}, \ldots, \chi_{s} ; \frac{\delta}{2}\right)-U\left(\chi_{1}, \ldots, \chi_{s} ; \frac{\delta}{2}\right) \subseteq U\left(\chi_{1}, \ldots, \chi_{s} ; \delta\right) .
$$

Corollary 4.31. For an abelian group $G$ all topologies of the form $\mathcal{T}_{H}$, where $H \leq G^{*}$, are totally bounded. Moreover, $\mathcal{T}_{H}$ is precompact iff $H$ separates the points of $G$.

It requires a considerable effort to prove that, conversely, every totally bounded group topology has the form $\mathcal{T}_{H}$ for some $H$ (see Remark 6.3).

It follows easily from Corollary 4.31 and Proposition 4.30 that for every neighborhood $E$ of 0 in the Bohr topology (namely, a set $E$ containing a subset of the form $U\left(\chi_{1}, \ldots, \chi_{n} ; \varepsilon\right.$ ) with characters $\chi_{i}: G \rightarrow \mathbb{S}$, $i=1,2, \ldots, n$, and $\varepsilon>0$ ) there exists a big set $B$ of $G$ such that $B_{(8)} \subseteq E$ (just take $B=U\left(\chi_{1}, \ldots, \chi_{n} ; \varepsilon / 8\right)$ ). Surprisingly, the converse is also true. Namely, we shall obtain as a corollary of Følner's lemma that every set $E$ satisfying $B_{(8)} \subseteq E$ for some big set $B$ of $G$ must be a neighborhood of 0 in the Bohr topology of $G$ (see Corollary 5.8).

Exercise 4.32. If $G$ is a countably infinite Hausdorff abelian group, then for every compact set $K$ in $G$ the set $K_{(2 n)}$ is big for no $n \in \mathbb{N}$.

(Hint. By Lemma 4.3 every set $K_{(2 n)}$ is compact. So if $K_{(2 n)}$ were big for some $n$, then $G$ itself would be compact. Now Lemma 4.8 applies.)

Exercise 4.33. Call a subset $S$ of an infinite abelian group $G$ small if there exist (necessarily distinct) elements $g_{1}, g_{2}, \ldots, g_{n}, \ldots$ of $G$ such that $\left(g_{n}+S\right) \cap\left(g_{m}+S\right)=\emptyset$ whenever $m \neq n$.

(a) Show that a subset $S$ of $G$ such that $S-S$ is not big is necessarily small.

(b) Show that every finite subset is small.

(c) Show that the group $\mathbb{Z}$ is not a finite union of small sets.

(d) * Show that no infinite abelian group $G$ is a finite union of small sets.

(e) If $S=\left(a_{n}\right)$ is a one-to-one $T$-sequence in an abelian group $G$, then for every $n \in \mathbb{N}$ the set $S_{(2 n)}$ is small in $G$.

(f) Show that the sequence $\left(p_{n}\right)$ of prime numbers in $\mathbb{Z}$ is not a $T$-sequence. 
(Hint. (d) Use a finitely additive invariant (Banach) measure on $G$. For (e) consider the (countable) subgroup generated by $S$ and note that if $a_{n} \rightarrow 0$ in some Hausdorff group topology $\tau$ on $G$, then the set $S \cup\{0\}$ would be compact in $\tau$, so item (a) and Exercise 4.32 apply. For (f) use (e) and the fact that there exists a constant $m$ such that every integer number is a sum of at most $m^{11}$ prime numbers.)

Exercise 4.34. Show that for an infinite abelian group $G$ and a subgroup $H$ of $G$ the following are equivalent:

(a) H has infinite index;

(b) $H$ is not big;

(c) $H$ is small.

Exercise 4.35. * Every infinite abelian group has a small set of generators.

This can be extended to arbitrary groups [30]. One can find in the literature also different (weaker) forms of smallness $([4,11])$.

Exercise 4.36. (a) If $f: G \rightarrow H$ is a continuous surjective homomorphism of topological groups, then $H$ is totally bounded whenever $G$ is totally bounded.

(b) Prove that a topological group $G$ is totally bounded iff $G / \overline{\{1\}}$ is totally bounded.

(c) If $\left\{G_{i}: i \in I\right\}$ is a family of topological groups, then $\prod_{i} G_{i}$ is totally bounded iff each $G_{i}$ is totally bounded.

(d) Prove that every topological abelian group $G$ admits a "universal" totally bounded continuous surjective homomorphic image $q: G \rightarrow q(G)$ (i.e., every continuous homomorphsm $G \rightarrow P$, where $P$ is a totally bounded group, factors through $\left.q^{12}\right)$.

\subsection{Subgroups of $\mathbb{R}^{n}$}

Our main goal here is to prove that every closed subgroup of $\mathbb{R}^{n}$ is topologically isomorphic to $\mathbb{R}^{s} \times \mathbb{Z}^{m}$, with $s, m \in \mathbb{N}$ and $s+m \leq n$. More precisely:

Theorem 4.37. Let $n \in \mathbb{N}_{+}$and let $H$ be a closed subgroup of $\mathbb{R}^{n}$. Then there exist closed subgroups $V$ and $D$ of $\mathbb{R}^{n}$ such that $H=V+D \cong V \times D, V \cong \mathbb{R}^{s}, D \cong \mathbb{Z}^{m}$ and $s+m \leq n$.

The proof is split in several steps. Before starting it, we note the following curious dichotomy hidden in this theorem:

- the closed connected subgroups of $\mathbb{R}^{n}$ are always isomorphic to some $\mathbb{R}^{s}$ with $s \leq n$;

- the totally disconnected closed subgroups $D$ of $\mathbb{R}^{n}$ must be free and have free-rank $r_{0}(D) \leq n$; in particular they are discrete.

In the general case, for every closed subgroup $H$ of $\mathbb{R}^{n}$ the connected component $c(H)$ is open in $H$ and isomorphic to $\mathbb{R}^{s}$ for some $s \leq n$. Therefore, by the divisibility of $\mathbb{R}^{s}$ one can write $H=c(H) \times D$ for some discrete subgroup $D$ of $H$ (see Corollary 2.8). Necessarily $r_{0}(D) \leq n-s$ as $c(H) \cong \mathbb{R}^{s}$ contains a discrete subgroup $D_{1}$ of rank $s$, so that $D_{1} \times D$ will be a discrete subgroup of $\mathbb{R}^{n}$.

It is not hard to see that every discrete subgroup of $\mathbb{R}$ is cyclic (Exercise 3.20 ). The first part of the proof consists in appropriately extending this property to discrete subgroups of $\mathbb{R}^{n}$ (see Proposition 4.39). The first step is to see that the free-rank $r_{0}(H)$ of a discrete subgroup $H$ of $\mathbb{R}^{n}$ coincides with the dimension of the subspace of $\mathbb{R}^{n}$ generated by $H$.

Lemma 4.38. Let $H$ be a discrete subgroup of $\mathbb{R}^{n}$. If the elements $v_{1}, \ldots, v_{m}$ of $H$ are $\mathbb{Q}$-linearly independent, then they are also $\mathbb{R}$-linearly indipendent.

\footnotetext{
${ }^{11}$ use the fact that according to the positive solution of the ternary Goldbach's conjecture a there exists a constant $C>0$ such that every odd integer $\geq C$ is a sum of three primes (see [96] for further details on Goldbach's conjecture).

12 in other words, the subcategory of all totally bounded groups forms an epireflective subcategory of the category of all topological groups.
} 
Proof. Let $V \cong \mathbb{R}^{k}$ be the subspace of $\mathbb{R}^{n}$ generated by $H$. We can assume wlog that $V=\mathbb{R}^{n}$, i.e., $k=n$. Hence we have to prove that the free-rank $m=r_{0}(H)$ of $H$ coincides with $n$. Obviously $m \geq n$. We need to prove that $m \leq n$. Let us fix $n \mathbb{R}$-linearly independent vectors $v_{1}, \ldots, v_{n}$ in $H$. It is enough to see that for every $h \in H$ the vectors $v_{1}, \ldots, v_{n}, h$ are not $\mathbb{Q}$-linearly independent. This would imply $m \leq n$. Let us note first that we can assume wlog that $H \supseteq \mathbb{Z}^{n}$. Indeed, as $v_{1}, \ldots, v_{n}$ are $\mathbb{R}$-linearly independent, there exists a linear isomorphism $\alpha: \mathbb{R}^{n} \rightarrow \mathbb{R}^{n}$ with $\alpha\left(v_{i}\right)=e_{i}$ for $i=1,2, \ldots, n$, where $e_{1}, \ldots, e_{n}$ is the canonical base of $\mathbb{R}^{n}$. Clearly, $\alpha(H)$ is still a discrete subgroup of $\mathbb{R}^{n}$ and the vectors $v_{1}, \ldots, v_{n}, h$ are $\mathbb{Q}$-linearly independent iff the vectors $e_{1}=\alpha\left(v_{1}\right), \ldots, e_{n}=\alpha\left(v_{n}\right), \alpha(h)$ are. The latter fact is equivalent to $\alpha(h) \notin \mathbb{Q}^{n}$. Therefore, arguing for a contradiction, assume for simplicity that $H \supseteq \mathbb{Z}^{n}$ and there exists $h=\left(h_{1}, \ldots, h_{n}\right) \in H$ such that

$$
h \notin \mathbb{Q}^{n} .
$$

By the discreteness of $H$ there exists an $\varepsilon>0$ with $\max \left\{\left|h_{i}\right|: i=1,2, \ldots, n\right\} \geq \varepsilon$ for every $0 \neq h=$ $\left(h_{1}, \ldots, h_{n}\right) \in H$. Represent the cube $C=[0,1]^{n}$ as a finite union $\bigcup_{i} C_{i}$ of cubes $C_{i}$ of diameter $<\varepsilon$ (e.g., take them with faces parallel to the coordinate axes, although their precise position is completely irrelevant). For a real number $r$ denote by $\{r\}$ the unique number $0 \leq x<1$ such that $r-x \in \mathbb{Z}$. Then $\left(\left\{m v_{1}\right\}, \ldots,\left\{m v_{n}\right\}\right) \neq$ $\left(\left\{l h_{1}\right\}, \ldots,\left\{l h_{n}\right\}\right)$ for every positive $l \neq m$, since otherwise, $(m-l) h \in \mathbb{Z}^{n}$ with $m-l \neq 0$ in contradiction with (4). Among the infinitely many points $a_{m}=\left(\left\{m h_{1}\right\}, \ldots,\left\{m h_{n}\right\}\right) \in C$ there exist two $a_{m} \neq a_{l}$ belonging to the same cube $C_{i}$. Hence, $\left|\left\{m h_{j}\right\}-\left\{l h_{j}\right\}\right|<\varepsilon$ for every $j=1,2, \ldots, n$. So there exists a $z=\left(z_{1}, \ldots, z_{n}\right) \in \mathbb{Z}^{n}$, such that $0 \neq(m-l) h-z \in H$ and $\left|(m-l) h_{j}-z_{j}\right|<\varepsilon$ for every $j=1,2, \ldots, n$, this contradicts the choice of $\varepsilon$.

The aim of the next step is to see that the discrete subgroups of $\mathbb{R}^{n}$ are free.

Proposition 4.39. If $H$ is a discrete subgroup of $\mathbb{R}^{n}$, then $H$ is free and $r(H) \leq n$.

Proof. In fact, let $m=r(H)$. By the definition of $r(H)$ there exist $m \mathbb{Q}$-linearly independent vectors $v_{1}, \ldots, v_{m}$ of $H$. By the previous lemma the vectors $v_{1}, \ldots, v_{m}$ are also $\mathbb{R}$-linearly independent. Hence, $m \leq n$. Let $V \cong \mathbb{R}^{m}$ be the subspace of $\mathbb{R}^{n}$ generated by $v_{1}, \ldots, v_{m}$. Obviously, $H \subseteq V$, since $H$ is contained in the $\mathbb{Q}$-subspace of $\mathbb{R}^{n}$ generated by the free subgroup $F=\left\langle v_{1}, \ldots, v_{m}\right\rangle$ di $H$. Since $H$ is a discrete subgroup of $V$ too, we can argue with $V$ in place of $\mathbb{R}^{n}$. So, we can assume wlog that $m=n$ and $V=\mathbb{R}^{n}$. It suffices to see that $H / F$ is finite. Then $H$ will be finitely generated and torsion-free, hence $H$ must be free.

Since the vectors $v_{1}, \ldots, v_{n}$ are linearly independent on $\mathbb{R}$ we can assume wlog that $H \supseteq \mathbb{Z}^{n}$. In fact, let $\alpha: \mathbb{R}^{n} \rightarrow \mathbb{R}^{n}$ be the linear isomorphism with $\alpha\left(v_{i}\right)=e_{i}$ for $i=1,2, \ldots, n$, where $e_{1}, \ldots, e_{n}$ is the canonical base of $\mathbb{R}^{n}$. Then $\alpha(H)$ is still a discrete subgroup of $\mathbb{R}^{n}, \mathbb{Z}^{n}=\alpha(F) \subseteq \alpha(H)$ and $H / F$ is finite iff $\alpha(H) / \alpha(F) \cong H / F$ is finite.

In the sequel we assume $H \supseteq \mathbb{Z}^{n}=F$ for the sake of simplicity. To check that $H / F$ is finite consider the canonical homomorphism $q: \mathbb{R}^{n} \rightarrow \mathbb{R}^{n} / \mathbb{Z}^{n} \cong \mathbb{T}^{n}$. According to Theorem 3.23, $q$ sends the closed subgroup $H$ onto a closed (hence compact) subgroup $q(H)$ of $\mathbb{T}^{n}$; moreover $H=q^{-1}(q(H))$, hence the restriction of $q$ to $H$ is open and $q(H)$ is discrete. Thus $q(H) \cong H / F$ is both compact and discrete, so $q(H)$ must be finite.

Now we are in position to prove Theorem 4.37. We advise the reader to review the warming exercise 3.20.

Proof of Theorem 4.37. Let $H \neq 0$ a closed subgroup of $\mathbb{R}^{n}$. If $H$ discrete, then $H$ is free and generates a linear subspace of $\mathbb{R}^{n}$ of dimension $r(H) \leq n$ by Proposition 4.39, so the assertion is true with $s=0$.

In case $H$ is not discrete we argue by induction on $n$. The case $n=1$ is Exercise 3.20 . Let $n>1$ and assume the theorem is true for $n-1$. Consider the subset

$$
M=\left\{u \in \mathbb{R}^{n}:\|u\|=1 \text { and } \exists \lambda \in(0,1) \text { with } \lambda u \in H\right\}
$$

of the unitary sphere $S$ in $\mathbb{R}^{n}$. For $u \in S$ let $N_{u}=\{r \in \mathbb{R}: r u \in H\}$. Then $N_{u}$ is a closed subgroup of $\mathbb{R}$ and $H \cap \mathbb{R} u=N_{u} u$. Our aim will be to find some $u \in S$ such that the whole line $\mathbb{R} u$ is contained in $H$. This will allow us to use our inductive hypothesis. Since the proper closed subgroups of $\mathbb{R}$ are cyclic (see Exercise 3.20 ), it suffices to find some $u \in S$ such that $N_{u}$ is not cyclic.

Case 1. If $M=\left\{u_{1}, \ldots, u_{n}\right\}$ is finite, then there exists an index $i$ such that $\lambda u_{i} \in H$ for infinitely many $\lambda \in(0,1)$. Then the closed subgroup $N_{u_{i}}$ cannot be cyclic, so $H$ contains to line $\mathbb{R} u_{i}$ and we are done.

Case 2. Assume $M$ is infinite. By the assumption $H$ is not discrete there exists a sequence $u_{n} \in M$ such that the corresponding $\lambda_{n}$, with $\lambda_{n} u_{n} \in H$, converge to 0 . By the compactness of $S$ there exists a limit point $u_{0} \in S$ for the sequence $u_{n} \in M$. We can assume wlog that $u_{n} \rightarrow u_{0}$. Let $\varepsilon>0$ and let $\Delta_{\varepsilon}$ be the open interval $(\varepsilon, 2 \varepsilon)$. As $\lambda_{n} \rightarrow 0$, there exists $n_{0}$ such that $\lambda_{n}<\varepsilon$ for every $n \geq n_{0}$. Hence for every $n \geq n_{0}$ there exists an 
appropriate $k_{n} \in \mathbb{N}$ with $\eta_{n}=k_{n} \lambda_{n} \in \Delta_{\varepsilon}$. Taking again a subsequence we can assume wlog that there exists some $\xi_{\varepsilon} \in \overline{\Delta_{\varepsilon}}$ such that $\eta_{n} \rightarrow \xi_{\varepsilon}$. Hence $\xi_{\varepsilon} u_{0}=\lim _{n} k_{n} \lambda_{n} u_{0} \in H$. This argument shows that $N_{u_{0}}$ contains $\xi_{\varepsilon} \in \overline{\Delta_{\varepsilon}}$ with arbitrarily small $\varepsilon$. Therefore, $N_{u_{0}}$ cannot be cyclic. Hence $H$ contains the line $\mathbb{R} u_{0}$.

We proved in all cases that our assumption of non-discreteness of $H$ yields the existence of a line $L \cong \mathbb{R}$ as a subgroup of $H$. Let $L^{\prime} \cong \mathbb{R}^{n-1}$ be a subspace of $\mathbb{R}^{n}$ complementing $L$. Then $\mathbb{R}^{n}=L \times L^{\prime}$ and the projection $\mathbb{R}^{n} \rightarrow L^{\prime}$ sends $H$ to a closed subgroup $H_{1}$ of $L^{\prime}$ as $L \leq H$ (cf. 3.23 (b)). Moreover, $H=L \times H_{1}$ in view of $L \leq H$ again. Now proceed by induction with the subgroup $H_{1}$ of $L^{\prime} \cong \mathbb{R}^{n-1}$. This proves Theorem 4.37.

The next corollary describes the quotients of $\mathbb{R}^{n}$.

Corollary 4.40. A quotient of $\mathbb{R}^{n}$ is isomorphic to $\mathbb{R}^{k} \times \mathbb{T}^{m}$, where $k+m \leq n$. In particular, a compact quotient of $\mathbb{R}^{n}$ is isomorphic to $\mathbb{T}^{m}$ for some $m \leq n$.

Proof. Let $H$ be a closed subgroup of $\mathbb{R}^{n}$. Then $H=V+D$, where $V, D$ are as in Theorem 4.37. If $s=\operatorname{dim} V$ and $m=r_{0}(D)$, then $s+m \leq n$. Let $V_{1}$ be the linear subspace of $\mathbb{R}^{n}$ spanned by $D$. Pick a complementing subspace $V_{2}$ for the subspace $V+V_{1}$ and let $k=n-(s+m)$. Then $\mathbb{R}^{n}=V+V_{1}+V_{2}$ is a factorization in direct product. Therefore $\mathbb{R}^{n} / H \cong\left(V_{1} / D\right) \times V_{2}$. Since $\operatorname{dim} V_{1}=r_{0}(D)=m$, one has $V_{1} / D \cong \mathbb{T}^{m}$. Therefore, $\mathbb{R}^{n} / H \cong \mathbb{T}^{m} \times \mathbb{R}^{k}$

Let us denote by $(x \mid y)$ the usual scalar product in $\mathbb{R}^{n}$. Recall that every base $v_{1}, \ldots, v_{n}$ di $\mathbb{R}^{n}$ admits a dual base $v_{1}^{\prime}, \ldots, v_{n}^{\prime}$ defined by the relations $\left(v_{i} \mid v_{j}^{\prime}\right)=\delta_{i j}$. For a subgroup $H$ of $\mathbb{R}^{n}$ define the associated subgroup $H^{\dagger}$ setting

$$
H^{\dagger}:=\left\{u \in \mathbb{R}^{n}:(\forall x \in H)(x \mid u) \in \mathbb{Z}\right\}
$$

Then obviously $\left(\mathbb{Z}^{n}\right)^{\dagger}=\mathbb{Z}^{n}$.

Lemma 4.41. Let $H$ be a subgroup di $\mathbb{R}^{n}$. Then:

1. $H^{\dagger}$ is a closed subgroup of $\mathbb{R}^{n}$ and the correspondence $H \mapsto H^{\dagger}$ is decreasing;

2. $(\bar{H})^{\dagger}=H^{\dagger}$.

Proof. The map $\mathbb{R}^{n} \times \mathbb{R}^{n} \rightarrow \mathbb{R}$ defined by $(x, y) \mapsto(x \mid u)$ is continuous.

(a) Let $q: \mathbb{R} \rightarrow \mathbb{T}=\mathbb{R} / \mathbb{Z}$ be the canonical homomorphism. Then for every fixed $a \in \mathbb{R}^{n}$ the assignment $x \mapsto(a \mid x) \mapsto f((a \mid x))$ is a continuous homomorphism $\chi_{a}: \mathbb{R}^{n} \rightarrow \mathbb{T}$. Hence the set $\chi_{h}^{-1}(0)=\left\{u \in \mathbb{R}^{n}:(\forall h \in\right.$ $H)(h \mid u) \in \mathbb{Z}\}$ is closed in $\mathbb{R}^{n}$. Therefore $H^{\dagger}=\bigcap_{h \in H} \chi_{h}^{-1}(0)$ is closed. The same equality proves that the correspondence $H \mapsto H^{*}$ is decreasing.

(b) From the second part of (a) one has $(\bar{H})^{\dagger} \subseteq H^{\dagger}$. Suppose that $u \in H^{\dagger}$ e $x \in \bar{H}$. By the continuity of the map $\chi_{x}(u)=\chi_{u}(x)$, as a function of $x$, one can deduce that $\chi_{x}(u)=0$, being $\chi_{u}(h)=0$ for every $h \in H$.

We study in the sequel the subgroup $H^{\dagger}$ associated to a closed subgroup $H$ of $\mathbb{R}^{n}$. According to Theorem 4.37 there exist a base $v_{1}, \ldots, v_{n}$ of $\mathbb{R}^{n}$ and $k \leq n$, such that for some $0 \leq s \leq k H=V \oplus L$ where $V$ is the linear subspace generated by $v_{1}, \ldots, v_{s}$ and $L=\left\langle v_{s+1}, \ldots, v_{k}\right\rangle$. Let $v_{1}^{\prime}, \ldots, v_{n}^{\prime}$ be the dual base of $v_{1}, \ldots, v_{n}$.

Lemma 4.42. In the above notation the subgroup $H^{\dagger}$ coincides with $\left\langle v_{s+1}^{\prime}, \ldots, v_{k}^{\prime}\right\rangle+W$, where $W$ is the linear subspace generated by $v_{k+1}^{\prime}, \ldots, v_{n}^{\prime}$.

Proof. Let $V^{\prime}$ be the linear subspace generated by $v_{1}^{\prime}, \ldots, v_{s}^{\prime}, V^{\prime \prime}$ the linear subspace generated by $v_{s+1}^{\prime}, \ldots, v_{k}^{\prime}$ and $L^{\prime}=\left\langle v_{s+1}^{\prime}, \ldots, v_{k}^{\prime}\right\rangle$. Then $L^{\dagger}=V^{\prime}+L^{\prime}+W$, while $V^{\dagger}=V^{\prime \prime}+W$. Hence $H^{\dagger} \leq L^{\dagger} \cap V^{\dagger}=L^{\prime}+W$. On the other hand, obviously $L^{\prime}+W \leq H^{\dagger}$.

Corollario 4.43. $\bar{H}=\left(H^{\dagger}\right)^{\dagger}$ for every subgroup $H$ of $\mathbb{R}^{n}$.

Proof. If $H$ is closed of the form $V+L$ in the notation of the previous lemma, then $H^{\dagger}=L^{\prime}+W$ with $v_{1}^{\prime}, \ldots, v_{n}^{\prime}$, $L^{\prime}$ and $W$ defined as above. Now $H^{\dagger}=L^{\prime}+W$ is a closed subgroup of $\mathbb{R}^{n}$ by Lemma 4.41 and $v_{1}, \ldots, v_{n}$ is a dual base of $v_{1}^{\prime}, \ldots, v_{n}^{\prime}$. Therefore, $H=V+L$ coincides with $\left(H^{\dagger}\right)^{\dagger}$.

Lemma 4.44. Let $V$ be a hyperplain in $\mathbb{R}^{n}$ determined by the equation $\sum_{i=1}^{n} a_{i} x_{i}=0$ such that there exists at least one coefficient $a_{i}=1$. Then the subgroup $H=V+\mathbb{Z}^{n}$ of $\mathbb{R}^{n}$ is not dense iff all the coefficients $a_{i}$ are rational.

Proof. We can assume wlog that $i=n$. Suppose that $H$ is not dense in $\mathbb{R}^{n}$. Then $H^{\dagger} \neq 0$ by Corollary 4.43. Let $0 \neq z \in H^{\dagger}$. Since $\mathbb{Z}^{n} \leq H$, one has $H^{\dagger} \leq \mathbb{Z}^{n}=\left(\mathbb{Z}^{n}\right)^{\dagger}$, so $z \in \mathbb{Z}^{n}$. If $j<n$, then $a_{j} \in \mathbb{Q}$ as $v=\left(0, \ldots, 0,1,0, \ldots, 0,-a_{j}\right) \in V$ 
The next proposition is a particular case of the well-known Kronecker's theorem.

Proposition 4.45. Let $v_{1}, \ldots, v_{n} \in \mathbb{R}$. Then for $v=\left(v_{1}, \ldots, v_{n}\right) \in \mathbb{R}^{n}$ the subgroup $\langle v\rangle+\mathbb{Z}^{n}$ of $\mathbb{R}^{n}$ is dense iff $v_{0}=1, v_{1}, \ldots, v_{n} \in \mathbb{R}$ are linearly independent as elements of the vector space $\mathbb{R}$ over $\mathbb{Q}$.

Proof. Assume $v_{0}=1, v_{1}, \ldots, v_{n} \in \mathbb{R}$ are linearly independent and let $H=\langle v\rangle+\mathbb{Z}^{n}$. Then $H^{\dagger} \subseteq \mathbb{Z}^{n}=\left(\mathbb{Z}^{n}\right)^{\dagger}$. It is easy to see now that some $z \in \mathbb{Z}^{n}$ belongs to $(\langle v\rangle)^{\dagger}$ iff $z=0$. This proves that $H^{\dagger}=0$. Consequently $H$ is dense in $\mathbb{R}^{n}$ by Corollary 4.43. If $\sum_{i=0}^{n} k_{i} v_{i}=0$ is a non-trivial linear combination with $k_{i} \in \mathbb{Z}$, then the vector $k=\left(k_{1}, \ldots, k_{n}\right) \in \mathbb{Z}^{n}$ is non-zero and obviously $k \in H^{\dagger}$. Thus $H^{\dagger} \neq 0$, hence $H$ is not dense.

Theorem 4.46. $\mathbb{T}^{\mathfrak{c}}$ is monothetic.

Proof. Let $B$ be a Hamel base of $\mathbb{R}$ on $\mathbb{Q}$ that contains 1 and let $B_{0}=B \backslash\{1\}$. Applying the previous proposition one can see that the element $x=\left(x_{b}\right)_{b \in B_{0}} \in \mathbb{T}^{B_{0}}$, defined by $x_{b}=b+\mathbb{Z} \in \mathbb{R} / \mathbb{Z}=\mathbb{T}$, is a generator of the group $\mathbb{T}^{B_{0}}$. To conclude note that $\left|B_{0}\right|=|\mathbb{R}|=\mathfrak{c}$.

Corollary 4.47. Let $C$ be a closed subgroup of $\mathbb{T}^{n}$. Then $C$ is isomorphic to $\mathbb{T}^{s} \times F$ where $s \leq n$ and $F$ is a finite abelian group.

Proof. Let $q: \mathbb{R}^{n} \rightarrow \mathbb{T}^{n}=\mathbb{R}^{n} / \mathbb{Z}^{n}$ be the canonical projection. If $C$ is a closed subgroup of $\mathbb{T}^{n}$, then $H=q^{-1}(C)$ is a closed subgroup of $\mathbb{R}^{n}$ that contains $\mathbb{Z}^{n}=\operatorname{ker} q$. Hence $H$ is a direct product $H=V+D$ with $V \cong \mathbb{R}^{s}$ and $D \cong \mathbb{Z}^{m}$, where $s$ and $m$ satisfy $s+m=n$ as $\mathbb{Z}^{n} \leq H$. Since the restriction of $q$ to $H$ is open by Theorem 3.23 , we conclude that the restriction of $q$ to $V$ is open as far as $V$ is open in $H$. Hence $q \uparrow_{V}: V \rightarrow q(V)$ is an open surjective homomorphism and the subgroup $q(V)$ is open in $C$. Since $q(V)$ is also divisible, we can write $C=q(V) \times F$, where the subgroup $F$ of $C$ must be discrete. Since $C$ is compact, this implies that $F$ is finite. On the other hand, as a compact quotient of $V \cong \mathbb{R}^{s}$ the group $q(V)$ is isomorphic to $\mathbb{T}^{s}$ by Corollary 4.40. Therefore, $C \cong \mathbb{T}^{s} \times F$.

Exercise 4.48. Determine for which of the following possible choices of the vector $v \in \mathbb{R}^{4}$

$$
(\sqrt{2}, \sqrt{3}, \sqrt{5}, \sqrt{6}),(\sqrt{2}, \sqrt{3}, \sqrt{5}, \sqrt{7}),(\log 2, \log 3, \log 5, \log 6),
$$

$(\log 2, \log 3, \log 5, \log 7),(\log 3, \log 5, \log 7, \log 9)$ and $(\log 5, \log 7, \log 9, \log 11)$

the subgroup $\langle v\rangle+\mathbb{Z}^{4}$ of $\mathbb{R}^{4}$ is dense.

Exercise 4.49. (a) Prove that a subgroup $H$ of $\mathbb{T}$ is dense iff $H$ is infinite.

(b) Determine the minimal (w.r.t. inclusion) dense subgroups $\mathbb{T}$.

(c)* Determine the minimal (w.r.t. inclusion) dense subgroups $\mathbb{T}^{2}$.

\section{Følner's theorem}

This section is entirely dedicated to Følner's theorem.

\subsection{Fourier theory for finite abelian groups}

In the sequel $G$ will be a finite abelian group, so $G^{*} \cong G$, so in particular $\left|G^{*}\right|=|G|$.

Here we recall some well known properties of the scalar product in finite-dimensional complex spaces $V=\mathbb{C}^{n}$. Since our space will be "spanned" by a finite abelian group $G$ of size $n$ (i.e., $V=\mathbb{C}^{G}$ ), we have also an action of $G$ on $V$. We normalize the scalar product in a such way to let the vector $(1,1, \ldots, 1)$ (i. e., the constant function 1) to have norm 1 . The reader familiar with Haar integration may easily recognize in this the Haar integral on $G$.

Define the scalar product by

$$
(f, g)=\frac{1}{|G|} \sum_{x \in G} f(x) \overline{g(x)} .
$$

Let us see first that the elements of the subset $G^{*}$ of $V$ are pairwise orthogonal and have norm 1:

Proposition 5.1. Let $G$ be an abelian finite group and $\varphi, \chi \in G^{*}, x, y \in G$. Then: 
(a) $\frac{1}{|G|} \sum_{x \in G} \varphi(x) \overline{\chi(x)}=\left\{\begin{array}{ll}1 & \text { if } \varphi=\chi \\ 0 & \text { if } \varphi \neq \chi\end{array} ;\right.$

(b) $\frac{1}{\left|G^{*}\right|} \sum_{\chi \in G^{*}} \chi(x) \overline{\chi(y)}=\left\{\begin{array}{ll}1 & \text { if } x=y \\ 0 & \text { if } x \neq y\end{array}\right.$.

Proof. (a) If $\varphi=\chi$ then $\chi(x) \overline{\chi(x)}=\chi(x) \chi(x)^{-1}=1$.

If $\varphi \neq \chi$ there exists $z \in G$ such that $\varphi(z) \neq \chi(z)$. Therefore the following equalities

$$
\sum_{x \in G} \varphi(x) \overline{\chi(x)}=\frac{\varphi(z)}{\chi(z)} \sum_{x \in G} \varphi(x-z) \overline{\chi(x-z)}=\frac{\varphi(z)}{\chi(z)} \sum_{x \in G} \varphi(x) \overline{\chi(x)}
$$

imply that $\sum_{x \in F} \varphi(x) \overline{\chi(x)}=0$.

(b) If $x=y$ then $\chi(x) \overline{\chi(x)}=\chi(x) \chi(x)^{-1}=1$.

If $x \neq y$, by Corollary 2.7 there exists $\chi_{0} \in G^{*}$ such that $\chi_{0}(x) \neq \chi_{0}(y)$. Now we can proceed as before, that is

$$
\sum_{\chi \in G^{*}} \chi(x) \overline{\chi(y)}=\frac{\chi_{0}(x)}{\chi_{0}(y)} \sum_{\chi \in G^{*}}\left(\chi \chi_{0}\right)(x) \overline{\left(\chi \chi_{0}\right)(y)}=\frac{\chi_{0}(x)}{\chi_{0}(y)} \sum_{\chi \in G^{*}} \chi(x) \overline{\chi(y)}
$$

yields $\sum_{\chi \in G^{*}} \chi(x) \overline{\chi(y)}=0$.

If $G$ is a finite abelian group and $f$ is a complex valued function on $G$, then for every $\chi \in G^{*}$ we can define

$$
c_{\chi}=(f, \chi)=\frac{1}{|G|} \sum_{x \in G} f(x) \overline{\chi(x)}
$$

that is the Fourier coefficient of $f$ corresponding to $\chi$.

For complex valued functions $f, g$ on a finite abelian group $G$ define the convolution $f * g$ by $(f * g)(x)=$ $\frac{1}{|G|} \sum_{y \in G} \overline{f(y)} g(x+y)$.

Proposition 5.2. Let $G$ be an abelian finite group and $f$ a complex valued function on $G$ with Fourier coefficients $c_{\chi}$ where $\chi \in G^{*}$. Then:

(a) $f(x)=\sum_{\chi \in G^{*}} c_{\chi} \chi(x)$ for every $x \in G$;

(b) if $\left\{a_{\chi}\right\}_{\chi \in G^{*}}$ is such that $f(x)=\sum_{\chi \in G^{*}} a_{\chi} \chi(x)$, then $a_{\chi}=c_{\chi}$ for every $\chi \in G^{*}$;

(c) $\frac{1}{|G|} \sum_{x \in G}|f(x)|^{2}=\sum_{\chi \in G^{*}}\left|c_{\chi}\right|^{2}$;

(d) if $g$ is an other complex valued function on $G$ with Fourier coefficients $\left(d_{\chi}\right)_{\chi \in G^{*}}$, then $f * g$ has Fourier coefficients $\left(\overline{c_{\chi}} d_{\chi}\right)_{\chi \in G^{*}}$.

Proof. (a) The definition of the coefficients $c_{\chi}$ yields

$$
\sum_{\chi \in G^{*}} c_{\chi} \chi(x)=\sum_{\chi \in G^{*}} \frac{1}{|G|} \sum_{y \in G} f(y) \overline{\chi(y)} \chi(x) .
$$

Computing $\sum_{\chi \in G^{*}} \overline{\chi(y)} \chi(x)$ with Proposition 5.1(b) we get $\sum_{\chi \in G^{*}} c_{\chi} \chi(x)=\frac{\left|G^{*}\right|}{|G|} f(x)$ for every $x \in G$. Now $|G|=\left|G^{*}\right|$ gives $f(x)=\sum_{\chi \in G^{*}} c_{\chi} \chi(x)$ for every $x \in G$.

(b) By Proposition 5.1 the definition of the coefficients $c_{\chi}$ and the relation $f(x)=\sum_{\chi \in G^{*}} a_{\chi} \chi(x)$

$$
c_{\chi}=\frac{1}{|G|} \sum_{\varphi \in G^{*}} a_{\varphi} \sum_{x \in G} \varphi(x) \overline{\chi(x)}=a_{\chi} .
$$

(d) By item (a) $g(x)=\sum_{\varphi \in G^{*}} d_{\varphi} \varphi(x)$ for every $x \in G$. Therefore

$$
\begin{aligned}
\sum_{y \in G} \overline{f(y)} g(x+y) & =\sum_{y \in G}\left(\sum_{\chi \in G^{*}} \overline{c_{\chi}} \overline{\chi(y)}\right)\left(\sum_{\varphi \in G^{*}} d_{\varphi} \varphi(x) \varphi(y)\right)= \\
& =\sum_{\chi \in G^{*}} \sum_{\varphi \in G^{*}} \overline{c_{\chi}} d_{\varphi} \varphi(x) \sum_{y \in G} \overline{\chi(y)} \varphi(y)=|G| \sum_{\chi \in G^{*}} \overline{c_{\chi}} d_{\chi} \chi(x) .
\end{aligned}
$$

(c) It is sufficient to apply (d) with $g=f$ and let $x=0$. 
Corollary 5.3. Let $G$ be a finite abelian group, $E$ be a non-empty subset of $G$ and let $f$ be the characteristic function of $E$. Then for the convolution $g=f * f$ one has

(a) $g(x)>0$ iff $x \in E_{(2)}$;

(b) $g(x)=\sum_{\chi \in G^{*}}\left|c_{\chi}\right|^{2} \chi(x)$.

Proof. (a) $g(x)>0$ if and only if there exists $y \in E$ with $x+y \in E$, that is $x \in E-E=E_{(2)}$.

(b) follows obviously from Proposition 5.2(d).

\subsection{Bogoliouboff and Følner Lemmas}

Lemma 5.4 (Bogoliouboff lemma). If $F$ is a finite abelian group and $E$ is a non-empty subset of $F$, then there exist $\chi_{1}, \ldots, \chi_{m} \in F^{*}$, where $m=\left[\left(\frac{|F|}{|E|}\right)^{2}\right]$, such that $U\left(\chi_{1}, \ldots, \chi_{m} ; \frac{\pi}{2}\right) \subseteq E_{(4)}$.

Proof. Let $f$ be the characteristic function of E. By Proposition 5.2(a) we have

$$
f(x)=\sum_{\chi \in F^{*}} c_{\chi} \chi(x), \text { with } c_{\chi}=\frac{1}{|F|} \sum_{x \in F} f(x) \overline{\chi(x)} .
$$

Define $g=f * f$ and $h=g * g$. The functions $f$ and $g=f * f$ have real values and by Corollary 5.3

$$
g(x)=\sum_{\chi \in F^{*}}\left|c_{\chi}\right|^{2} \chi(x) \text { and } h(x)=\sum_{\chi \in F^{*}}\left|c_{\chi}\right|^{4} \chi(x) \text { for } x \in F .
$$

Moreover, $g(x)>0$ if and only if $x \in E-E=E_{(2)}$. Analogously $h(x)>0$ if and only if $x \in E_{(4)}$.

By Proposition 5.2(c) $\sum_{\chi \in F^{\prime}}\left|c_{\chi}\right|^{2}=\frac{|E|}{|F|}$. Set $a=\frac{|E|}{|F|}$ and order the Fourier coefficients of $f$ so that

$$
\left|c_{\chi_{0}}\right| \geq\left|c_{\chi_{1}}\right| \geq \ldots \geq\left|c_{\chi_{k}}\right| \geq \ldots
$$

(note that they are finitely many). Thus $\chi_{0}=1$ and $c_{\chi_{0}}=a$ by (1). Then $\sum_{i=0}^{k}\left|c_{\chi_{i}}\right|^{2} \leq \sum_{\chi \in F^{*}}\left|c_{\chi}\right|^{2}=a$ for every $k \geq 0$. Consequently $(k+1)\left|c_{\chi_{k}}\right|^{2} \leq a$, so

$$
\left|c_{\chi_{k}}\right|^{4} \leq \frac{a^{2}}{(k+1)^{2}} .
$$

Now let $m=\left[\frac{1}{a^{2}}\right]$. We are going to show now that with these $\chi_{1}, \ldots, \chi_{m} \in F^{*}$ one has

$$
h(x)>0 \text { for every } x \in U\left(\chi_{1} \ldots, \chi_{m} ; \frac{\pi}{2}\right) .
$$

Clearly $\operatorname{Re} \chi_{k}(x) \geq 0$ for $k=1,2, \ldots, m$ whenever $x \in U\left(\chi_{1} \ldots, \chi_{m} ; \frac{\pi}{2}\right)$ thus

$$
\left.\left|a^{4}+\sum_{k=0}^{m}\right| c_{\chi_{k}}\right|^{4} \chi_{k}(x) \mid \geq \operatorname{Re}\left(a^{4}+\sum_{k=1}^{m}\left|c_{\chi_{k}}\right|^{4} \chi_{k}(x)\right) \geq a^{4} .
$$

On the other hand, (3) yields

$$
\sum_{k \geq m+1}\left|c_{\chi_{k}}\right|^{4} \leq \sum_{k \geq m+1} \frac{a^{2}}{(k+1)^{2}}<a^{2} \sum_{k \geq m+1} \frac{1}{k(k+1)} \leq \frac{a^{2}}{m+1} .
$$

Since $h$ has real values, (2), (5) and (6) give

$h(x)=|h(x)|=\left.\left|a^{4}+\right| c_{\chi_{1}}\right|^{4} \chi_{1}(x)+\ldots|\geq| a^{4}+\left.\sum_{k=1}^{m}\left|c_{\chi_{k}}\right|^{4} \chi_{k}(x)\left|-\sum_{k \geq m+1}\right| c_{\chi_{k}}\right|^{4} \geq a^{4}-\frac{a^{2}}{m+1} \geq a^{2}\left(a^{2}-\frac{1}{m+1}\right)>0$.

This proves (4). Therefore $U\left(\chi_{1} \ldots, \chi_{m} ; \frac{\pi}{2}\right) \subseteq E_{(4)}$.

Let us note that the estimate for the number $m$ of characters is certainly non-optimal when $E$ is too small. For example, when $E$ is just the singleton $\{0\}$, the upper bound given by the lemma is just $|F|^{2}$, while one can certainly find at most $m=|F|-1$ characters $\chi_{1}, \ldots, \chi_{m}$ (namely, all non-trivial $\chi_{i} \in F^{*}$ ) such that $U\left(\chi_{1}, \ldots, \chi_{m} ; \frac{\pi}{2}\right)=\{0\}$. For certain groups (e.g., $F=\mathbb{Z}_{2}^{k}$ ) one can find even a much smaller number (say $\left.m=\log _{2}|F|\right)$. Nevertheless, in the cases relevant for the proof of Følner's theorem, namely when the subset $E$ is relatively large with respect to $F$, this estimate seems more reasonable.

The next lemma will be needed in the following proofs. 
Lemma 5.5. Let $A$ be an abelian group and $\left\{A_{n}\right\}_{n=1}^{\infty}$ be a sequence of finite subsets of $A$ such that

$$
\lim _{n \rightarrow \infty} \frac{\left|\left(A_{n}-a\right) \cap A_{n}\right|}{\left|A_{n}\right|}=1
$$

for every $a \in A$. If $k$ is a positive integer and $V$ is a subset of $A$ such that $k$ translates of $V$ cover $A$, then for every $\varepsilon>0$ there exists $N>0$ such that

$$
\left|V \cap A_{n}\right|>\left(\frac{1}{k}-\varepsilon\right)\left|A_{n}\right|
$$

for every $n \geq N$.

Proof. Let $a_{1}, \ldots, a_{k} \in A$ be such that $\bigcup_{i=1}^{k}\left(a_{i}+V\right)=A$. If $\varepsilon>0$, then there exists $N_{1}>0$ such that for every $n \geq N_{1}$

$$
\left|\left(A_{n}-a_{i}\right) \cap A_{n}\right|>(1-\varepsilon)\left|A_{n}\right|
$$

and consequently,

$$
\left|\left(A_{n}-a_{i}\right) \backslash A_{n}\right|<\varepsilon\left|A_{n}\right|
$$

for every $i=1, \ldots, k$. Since $A_{n}=\bigcup_{i=1}^{k}\left(a_{i}+V\right) \cap A_{n}$, for every $n$ there exists $i_{n} \in\{1, \ldots, k\}$ such that

$$
\frac{1}{k}\left|A_{n}\right| \leq\left|\left(a_{i_{n}}+V\right) \cap A_{n}\right|=\left|V \cap\left(A_{n}-a_{i_{n}}\right)\right| .
$$

Since $V \cap\left(A_{n}-a_{i_{n}}\right) \subseteq\left(V \cap A_{n}\right) \cup\left(\left(A_{n}-a_{i_{n}}\right) \backslash A_{n}\right),(7)$ yields

$$
\frac{1}{k}\left|A_{n}\right| \leq\left|V \cap\left(A_{n}-a_{i_{n}}\right)\right| \leq\left|V \cap A_{n}\right|+\left|\left(A_{n}-a_{i_{n}}\right) \backslash A_{n}\right|<\left|V \cap A_{n}\right|+\varepsilon\left|A_{n}\right| .
$$

Lemma 5.6 (Bogoliouboff-Følner lemma). Let $A$ be a finitely generated abelian group and let $r=r_{0}(A)$. If $k$ is a positive integer and $V$ is a subset of $A$ such that $k$ translates of $V$ cover $A$, then there exist $\rho_{1}, \ldots, \rho_{s} \in A^{*}$, where $s=3^{2 r} k^{2}$, such that $U_{A}\left(\rho_{1}, \ldots, \rho_{s} ; \frac{\pi}{2}\right) \subseteq V_{(4)}$.

Proof. By Theorem 2.1 we have $A \cong \mathbb{Z}^{r} \times F$, where $F$ is a finite abelian group; so we can identify $A$ with the group $\mathbb{Z}^{r} \times F$. Define $A_{n}=(-n, n]^{r} \times F$, let $a=\left(a_{1}, \ldots, a_{r} ; f\right) \in \mathbb{Z}^{r} \times F$. Then $J_{n i}=(-n, n] \cap\left(-n-a_{i}, n-a_{i}\right]$ satisfies $\left|J_{n i}\right| \geq 2 n-\left|a_{i}\right|$. In particular, $J_{n i} \neq \emptyset$ for every $n>n_{0}=\max \left\{\left|a_{i}\right|: i=1,2, \ldots, n\right\}$. As $\left(A_{n}-a\right) \cap A_{n}=$ $\prod_{i=1}^{r} J_{n i} \times F$, we have

$$
\left|\left(A_{n}-a\right) \cap A_{n}\right| \geq|F| \cdot \prod_{i=1}^{r}\left(2 n-\left|a_{i}\right|\right)
$$

or all $n>n_{0}$. Since $\left|A_{n}\right|=|F|(2 n)^{r}$, we can apply Lemma 5.5. Thus for every $\varepsilon>0$ we have

$$
\left|V \cap A_{n}\right|>\left(\frac{1}{k}-\varepsilon\right)\left|A_{n}\right|
$$

for every sufficiently large $n$. For $n$ with (8) define $G=A /\left(6 n \mathbb{Z}^{r}\right)$ and $E=q\left(V \cap A_{n}\right)$ where $q$ is the canonical projection of $A$ onto $G$. Observe that $q\left\lceil A_{n}\right.$ is injective, as $\left(A_{n}-A_{n}\right) \cap \operatorname{ker} q=\{0\}$. Then (8) gives

$$
|E|=\left|V \cap A_{n}\right|>\left(\frac{1}{k}-\varepsilon\right)\left|A_{n}\right|=\left(\frac{1}{k}-\varepsilon\right)(2 n)^{r}|F|
$$

and so

$$
\frac{|G|}{|E|} \leq \frac{(6 n)^{r}|F|}{\left(\frac{1}{k}-\varepsilon\right)(2 n)^{r}|F|}=\frac{3^{r} k}{1-k \varepsilon} .
$$

Fix $\varepsilon>0$ sufficiently small to have $\left[\frac{3^{2 r} k^{2}}{(1-k \varepsilon)^{2}}\right]=3^{2 r} k^{2}$ and pick sufficiently large $n$ to have (8). Now apply the Bogoliouboff Lemma 5.4 to find $s=3^{2 r} k^{2}$ characters $\xi_{1 n}, \ldots, \xi_{s n} \in G^{*}$ such that $U_{G}\left(\xi_{1 n}, \ldots, \xi_{s n} ; \frac{\pi}{2}\right) \subseteq E_{(4)}$. For $j=1, \ldots, s$ define $\varrho_{j n}=\xi_{j n} \circ \pi \in A^{*}$. If $a \in A_{n} \cap U_{A}\left(\varrho_{1 n}, \ldots, \varrho_{s n} ; \frac{\pi}{2}\right)$ then $q(a) \in U_{A}\left(\xi_{1 n}, \ldots, \xi_{s n} ; \frac{\pi}{2}\right) \subseteq E_{(4)}$ and so there exist $b_{1}, b_{2}, b_{3}, b_{4} \in V \cap A_{n}$ and $c=\left(c_{i}\right) \in 6 n \mathbb{Z}^{r}$ such that $a=b_{1}-b_{2}+b_{3}-b_{4}+c$. Now

$$
c=a-b_{1}+b_{2}-b_{3}+b_{4} \in\left(A_{n}\right)_{(4)}+A_{n}
$$


implies $\left|c_{i}\right| \leq 5 n$ for each $i$. So $c=0$ as $6 n$ divides $c_{i}$ for each $i$. Thus $a \in V_{(4)}$ and so

$$
A_{n} \cap U_{A}\left(\varrho_{1 n}, \ldots, \varrho_{s n} ; \frac{\pi}{2}\right) \subseteq V_{(4)}
$$

for all $n$ satisfying (8).

By Lemma 4.2 there exist $\varrho_{1}, \ldots, \varrho_{s} \in A^{*}$ and a subsequence $\left\{n_{l}\right\}_{l}$ of $\{n\}_{n \in \mathbb{N}_{+}}$such that $\varrho_{i}(a)=\lim _{l} \varrho_{i n_{l}}(a)$ for every $i=1, \ldots, s$ and $a \in A$. We are going to prove now that

$$
U_{A}\left(\varrho_{1}, \ldots \varrho_{s} ; \frac{\pi}{2}\right) \subseteq V_{(4)} .
$$

Take $a \in U_{A}\left(\varrho_{1}, \ldots, \varrho_{s} ; \frac{\pi}{2}\right)$. Since $A=\bigcup_{l=k}^{\infty} A_{n_{l}}$ for every $k \in \mathbb{N}_{+}$, there exists $n_{0}$ satisfying $(8)$ and $a \in A_{n_{0}}$. As $\varrho_{i}(a)=\lim _{l} \varrho_{i n_{l}}(a)$ for every $i=1, \ldots, s$, we can pick $l$ to have $n_{l} \geq n_{0}$ and $\left|A r g\left(\varrho_{i n_{l}}(a)\right)\right|<\pi / 2$ for every $i=1, \ldots, s$, i.e., $a \in U_{A}\left(\varrho_{1 n_{l}}, \ldots, \varrho_{s n_{l}} ; \frac{\pi}{2}\right) \cap A_{n_{l}}$. Now $(9)$, applied to $n_{l}$, yields $a \in V_{(4)}$. This proves (10).

Our next aim is to eliminate the dependence of the number $m$ of characters on the free rank of the group $A$ in Bogoliouboff - Følner's lemma. The price to pay for this is taking $V_{(8)}$ instead of $V_{(4)}$.

Lemma 5.7 (Følner lemma). Let $A$ be an abelian group. If $k$ is a positive integer and $V$ be a subset of $A$ such that $k$ translates of $V$ cover $A$, then there exist $\chi_{1}, \ldots, \chi_{m} \in A^{*}$, where $m=k^{2}$, such that $U_{A}\left(\chi_{1}, \ldots, \chi_{m} ; \frac{\pi}{2}\right) \subseteq$ $V_{(8)}$.

Proof. We consider first the case when $A$ is finitely generated. Let $r=r_{0}(A)$. By Lemma 5.6 there exist $\varrho_{1}, \ldots, \varrho_{s} \in A^{*}$, where $s=3^{2 r} k^{2}$, such that

$$
U_{A}\left(\varrho_{1}, \ldots, \varrho_{s} ; \frac{\pi}{2}\right) \subseteq V_{(4)}
$$

Since it is finitely generated, we can identify $A$ with $\mathbb{Z}^{r} \times F$, where $F$ is a finite abelian group. For $t \in\{1, \ldots, r\}$ define a monomorphism $i_{t}: \mathbb{Z} \hookrightarrow A$ by letting

$$
i_{t}(n)=(\underbrace{0, \ldots, 0, n}_{t}, 0, \ldots, 0 ; 0) \in A .
$$

Then each $\kappa_{j t}=\varrho_{j} \circ i_{t}$, where $j \in\{1, \ldots, s\}, t \in\{1, \ldots, r\}$, is a character of $\mathbb{Z}$. By Proposition 4.30 the subset

$$
L=U_{\mathbb{Z}}\left(\left\{\kappa_{j t}: j=1, \ldots, s, t=1, \ldots, r\right\} ; \frac{\pi}{8 r}\right)
$$

of $\mathbb{Z}$ is infinite. Let $L^{0}=\bigcup_{t=1}^{r} i_{t}(L)$, i.e., this is the set of all elements of $A$ of the form $\pm i_{t}(n)$ with $n \in L$ and $t \in\{1, \ldots, r\}$. Then obviously $L^{0}=-L^{0} \subseteq U_{A}\left(\varrho_{1}, \ldots, \varrho_{s} ; \frac{\pi}{8 r}\right)$, therefore,

$$
L_{(4 r)}^{0} \subseteq U_{A}\left(\varrho_{1}, \ldots, \varrho_{s} ; \frac{\pi}{2}\right) \subseteq V_{(4)}
$$

Define $A_{n}=(-n, n]^{r} \times F$ and pick $\varepsilon>0$ such that $\left[\left(\frac{k}{1-k \varepsilon}\right)^{2}\right]=k^{2}$. As in Lemma $5.6 A_{n}$ satisfies the hypotheses of Lemma 5.5 and so $\left|V \cap A_{n}\right|>\left(\frac{1}{k}-\varepsilon\right)\left|A_{n}\right|$ for sufficiently large $n$. Moreover, we choose this sufficiently large $n$ from $L$. Let $G_{n}=A /\left(2 n \mathbb{Z}^{r}\right) \cong \mathbb{Z}_{2 n}^{r} \times F$ and $E=q\left(A_{n} \cap V\right)$ where $q$ is the canonical projection $A \rightarrow G_{n}$. Then $q\left\lceil A_{n}\right.$ is injective as $\left(A_{n}-A_{n}\right) \cap \operatorname{ker} q=0$. So $q$ induces a bijection between $A_{n}$ and $G_{n}$ on one hand, and between $V \cap A_{n}$ and $E$. Thus $\left|A_{n}\right|=\left|G_{n}\right|=(2 n)^{r}|F|,|E|>\left(\frac{1}{k}-\varepsilon\right)\left|A_{n}\right|$ and so

$$
\left(\frac{\left|G_{n}\right|}{|E|}\right)^{2} \leq\left(\frac{k}{\varepsilon k-1}\right)^{2} \leq k^{2} .
$$

To the finite group $G_{n}$ apply the Bogoliouboff Lemma 5.4 to get $\xi_{1 n}, \ldots, \xi_{m n} \in G_{n}^{*}$, where $m=k^{2}$, such that

$$
U_{G_{n}}\left(\xi_{1 n}, \ldots, \xi_{m n} ; \frac{\pi}{2}\right) \subseteq E_{(4)}
$$

Let $\chi_{j n}=\xi_{j n} \circ q \in A^{*}$. If $a \in A_{n} \cap U_{A}\left(\chi_{1 n}, \ldots, \chi_{m n} ; \frac{\pi}{2}\right)$, then $q(a) \in U_{G_{n}}\left(\xi_{1 n}, \ldots, \xi_{m n} ; \frac{\pi}{2}\right) \subseteq E_{(4)}$. Therefore there exist $b_{1}, b_{2}, b_{3}, b_{4} \in A_{n} \cap V$ and $c=\left(c_{i}\right) \in 2 n \mathbb{Z}^{r}$ such that $a=b_{1}-b_{2}+b_{3}-b_{4}+c$. Since $2 n$ divides $c_{i}$ for every $i$ and $\left|c_{i}\right| \leq 5 n$, we conclude that $c_{i} \in\{0, \pm 2 n \pm 4 n\}$ for $i=1,2, \ldots, r$. This means that $c$ can be written as a sum of at most $4 r$ elements of $L^{0}$. This gives $c \in L_{(4 r)}^{0} \subseteq V_{(4)}$ by $(\lambda)$, consequently $a \in V_{(8)}$. Therefore

$$
A_{n} \cap U_{A}\left(\chi_{1 n}, \ldots, \chi_{m n} ; \frac{\pi}{2}\right) \subseteq V_{(8)}
$$


for $n \in L$ sufficiently large $n$. By Lemma 4.2 there exist $\chi_{1}, \ldots, \chi_{m} \in A^{*}$ and a subsequence $\left\{n_{l}\right\}_{l}$ of $\{n\}_{n \in \mathbb{N}_{+}}$ such that $\chi_{j}(a)=\lim _{l} \chi_{j n_{l}}(a)$ for every $j=1, \ldots, m$ and for every $a \in A$. Being $A=\bigcup\left\{A_{n}: l>k, n_{l} \in L\right\}$ for every $k \in \mathbb{N}_{+}$we can conclude as above that $U_{A}\left(\chi_{1}, \ldots, \chi_{m} ; \frac{\pi}{2}\right) \subseteq V_{(8)}$.

Consider now the general case. Let $g_{1}, \ldots, g_{k} \in A$ be such that $A=\bigcup_{i=1}^{k}\left(g_{i}+V\right)$. Suppose that $G$ is a finitely generated subgroup of $A$ containing $g_{1}, \ldots, g_{k}$. Then $G=\bigcup_{1=1}^{k}\left(g_{i}+V \cap G\right)$ and so $k$ translates of $V \cap G$ cover $G$. By the above argument and by Theorem 2.5 there exist $\varphi_{1 G}, \ldots, \varphi_{m G} \in G^{*}$, where $m=k^{2}$, such that

$$
U_{G}\left(\varphi_{1 G}, \ldots, \varphi_{m G} ; \frac{\pi}{2}\right) \subseteq(V \cap G)_{(8)} \subseteq V_{(8)}
$$

By Corollary 2.6 we can extend each $\varphi_{i G}$ to a character of $A$, so that we assume from now on $\varphi_{1 G}, \ldots, \varphi_{m G} \in A^{*}$ and

$$
G \cap U_{A}\left(\varphi_{1 G}, \ldots, \varphi_{m G} ; \frac{\pi}{2}\right)=U_{G}\left(\varphi_{1 G}, \ldots, \varphi_{m G} ; \frac{\pi}{2}\right) \subseteq V_{(8)} .
$$

Let $\mathcal{G}$ be the family of all finitely generated subgroups $G$ of $A$ containing $g_{1}, \ldots, g_{k}$. It is a directed set under inclusion. So we get $m$ nets $\left\{\varphi_{j G}\right\}_{G \in \mathcal{G}}$ in $A^{*}$ for $j=1, \ldots, m$. By Lemma 4.2 there exist subnets $\left\{\varphi_{j G_{\beta}}\right\}_{\beta}$ and $\chi_{1}, \ldots, \chi_{m} \in A^{*}$ such that

$$
\varphi_{j}(x)=\lim _{\beta} \varphi_{j G_{\beta}}(x) \text { for every } x \in A \text { and } j=1, \ldots, m \text {. }
$$

From (11) and (12) we conclude as before that $U_{A}\left(\chi_{1}, \ldots, \chi_{m} ; \frac{\pi}{2}\right) \subseteq V_{(8)}$.

As a corollary of Følner's lemma we obtain the following description of the neighborhoods of 0 in the Bohr topology of $A$.

Corollary 5.8. For a subset $E$ of an abelian group $A$ the following are equivalent:

(a) E contains $V_{(8)}$ for some big subset $V$ of $A$;

(b) for every $n \in \mathbb{N}_{+} E$ contains $V_{(2 n)}$ for some big subset $V$ of $A$;

(c) E is a neighborhood of 0 in the Bohr topology of $A$.

Proof. The implication (c) $\Rightarrow$ (b) follows from Følner's lemma. The implication (c) $\Rightarrow$ (b) follows from Corollary 4.31 and Proposition 4.30.

It follows from results of Følner [45] obtained by less elementary tools, that (b) can be replaced by the weaker assumption $V_{(4)} \subseteq E$ (see also Ellis and Keynes [43] or Cotlar and Ricabarra [24] for further improvements). Nevertheless the following old problems concerning the group $\mathbb{Z}$ is still open (see Cotlar and Ricabarra [24], Ellis and Keynes [43], Følner [45], Glasner [54], Pestov [80, Question 1025] or Veech [94]):

Question 5.9. Does there exist a big set $V \subseteq \mathbb{Z}$ such that $V-V$ is not a neighborhood of 0 in the Bohr topology of $G$ ?

It is known that every infinite abelian group $G$ admits a big set with empty interior with respect to the Bohr topology [4] (more precisely, these authors prove that every totally bounded group has a big subset with empty interior).

\subsection{Prodanov's lemma and proof of Følner's theorem}

Let $C$ be a set in a real or complex vector space. Then $C$ is said to be convex if, for all $x, y \in C$ and all $t \in[0,1]$, the point $(1-t) x+t y \in C$.

The next lemma, due to Prodanov [84], allows us to eliminate the discontinuous characters in uniform approximations of continuous functions via linear combinations of characters.

Lemma 5.10 (Prodanov's lemma). Let $G$ be a topological abelian group, let $U$ be an open subset of $G$, $f$ a complex valued continuous function on $G$ and $M$ a convex closed subset of $\mathbb{C}$. Let $k \in \mathbb{N}_{+}$and $\chi_{1}, \ldots, \chi_{k} \in G^{\prime}$. Suppose that $c_{1}, \ldots, c_{k} \in \mathbb{C}$ are such that $\sum_{j=1}^{k} c_{j} \chi_{j}(x)-f(x) \in M$ for every $x \in U$. If $\chi_{m_{1}}, \ldots, \chi_{m_{s}}$, with $m_{1}<\cdots<m_{s}, s \in \mathbb{N},\left\{m_{1}, \ldots, m_{s}\right\} \subseteq\{1, \ldots, k\}$, are the continuous among $\chi_{1}, \ldots, \chi_{k}$, then $\sum_{i=1}^{s} c_{m_{i}} \chi_{m_{i}}(x)-$ $f(x) \in M$ for every $x \in U$. 
Proof. Let $\chi_{k} \in G^{*}$ be discontinuous. Then it is discontinuous at 0 . Consequently there exists a net $\left\{x_{\gamma}\right\}_{\gamma}$ in $G$ such that $\lim _{\gamma} x_{\gamma}=0$ and there exist $y_{j}=\lim _{\gamma} \chi_{j}\left(x_{\gamma}\right)$ for all $j=1, \ldots, k$, but $y_{k} \neq 1$. Notice that always $\left|y_{j}\right|=1$. Moreover, $y_{j}=1$ when $\chi_{j}$ is continuous because $x_{\gamma} \rightarrow 0$, so $y_{j}=\lim \chi_{j}\left(x_{\gamma}\right)=1$.

Consider $\sum_{j=1}^{k} c_{j} \chi_{j}\left(x+t x_{\gamma}\right)-f\left(x+t x_{\gamma}\right)$, where $t \in \mathbb{Z}$. Since $\lim _{\gamma} x_{\gamma}=0$, we have $x+t x_{\gamma} \in U$ for every $x \in U$ and for every sufficiently large $\gamma$. Thus $\sum_{j=1}^{k} c_{j} \chi_{j}(x) \chi_{j}\left(x_{\gamma}\right)^{t}-f\left(x+t x_{\gamma}\right) \in M$ and so passing to the limit $\sum_{j=1}^{k} c_{j} \chi_{j}(x) y_{j}^{t}-f(x) \in M$, because $f$ is continuous and $M$ is closed.

Take an arbitrary $n \in \mathbb{N}$. By the convexity of $M$ and the relation above for $t=0, \ldots, n$, we obtain

$$
\frac{1}{n+1} \sum_{t=0}^{n}\left(\sum_{j=1}^{k} c_{j} \chi_{j}(x) y_{j}^{t}-f(x)\right) \in M .
$$

Note that $\sum_{t=0}^{n} y_{k}^{t}=\frac{y_{k}^{n+1}-1}{y_{k}-1}$ because $y_{k} \neq 1$. Hence we get

$$
\sum_{j=1}^{k-1} c_{j n} \chi_{j}(x)+\frac{c_{k}}{1+n} \frac{1-y_{k}^{n+1}}{1-y_{k}} \chi_{k}(x)-f(x) \in M
$$

for every $x \in U$, where $c_{j n}=\frac{\sum_{t=0}^{n} c_{j} y_{j}^{t}}{n+1}$. Now for every $j=1,2, \ldots, k-1$

- $\left|c_{j n}\right| \leq\left|c_{j}\right| \frac{\sum_{t=0}^{n}\left|y_{j}\right|^{t}}{n+1}=\left|c_{j}\right|$ (because $\left|y_{j}\right|=1$ ), and

- if $y_{j}=1$ then $c_{j n}=c_{j}$.

By the boundedness of the sequences $\left\{c_{j n}\right\}_{n=1}^{\infty}$ for $j=1, \ldots, k-1$, there exists a subsequence $\left\{n_{m}\right\}_{m=1}^{\infty}$ such that all limits $c_{j}^{\prime}=\lim _{m} c_{j n_{m}}$ exist for $j=1, \ldots, k-1$. On the other hand, $\left|y_{k}\right|=1$, so

$$
\lim _{n} \frac{c_{k}}{n+1} \frac{1-y_{k}^{n+1}}{1-y_{k}}=0 .
$$

Taking the limit for $m \rightarrow \infty$ in

$$
\sum_{j=1}^{k-1} c_{j n_{m}} \chi_{j}(x)+\frac{c_{k}}{1+n_{m}} \frac{1-y_{k}^{n_{m}+1}}{1-y_{k}} \chi_{k}(x)-f(x) \in M
$$

gives

$$
\sum_{j=1}^{k-1} c_{j}^{\prime} \chi_{j}(x)-f(x) \in M \text { for } x \in U
$$

moreover $c_{j}^{\prime}=c_{j}$ for every $j=1, \ldots, k-1$ such that $\chi_{j}$ is continuous.

The condition (13) is obtained by the hypothesis, removing the discontinuous character $\chi_{k}$ in such a way that the coefficients of the continuous characters remain the same. Iterating this procedure, we can remove all discontinuous characters among $\chi_{1}, \ldots, \chi_{k}$.

Now we give an (apparently) topology-free form of the local version of the Stone-Weierstraß theorem 2.19.

Proposition 5.11. Let $G$ be an abelian group and $H$ be a group of characters of $G$. If $X$ is a subset of $G$ and $f$ is a complex valued bounded function on $X$ then the following conditions are equivalent:

(a) $f$ can be uniformly approximated on $X$ by a linear combination of elements of $H$ with complex coefficients;

(b) for every $\varepsilon>0$ there exist $\delta>0$ and $\chi_{1}, \ldots, \chi_{m} \in H$ such that $x-y \in U_{G}\left(\chi_{1}, \ldots, \chi_{m} ; \delta\right)$ yields $|f(x)-f(y)|<\varepsilon$ for every $x, y \in X$.

Proof. (a) $\Rightarrow$ (b) Let $\varepsilon>0$. By (a) there exist $c_{1}, \ldots, c_{m} \in \mathbb{C}$ and $\chi_{1}, \ldots, \chi_{m} \in H$ such that $\left\|\sum_{i=1}^{m} c_{1} \chi_{i}-f\right\|_{\infty}<$ $\frac{\varepsilon}{4}$, that is $\left|\sum_{i=1}^{m} c_{1} \chi_{i}(x)-f(x)\right|<\frac{\varepsilon}{4}$ for every $x \in X$.

On the other hand note that $\left|\sum_{i=1}^{m} c_{i} \chi_{i}(x)-\sum_{i=1}^{m} c_{i} \chi_{i}(y)\right| \leq \sum_{i=1}^{m}\left|c_{i}\right| \cdot\left|\chi_{i}(x)-\chi_{i}(y)\right|$ and that $\mid \chi_{i}(x-$ $y)-1|=| \chi_{i}(x) \chi_{i}(y)^{-1}-1|=| \chi_{i}(x)-\chi_{i}(y) \mid$. If we take

$$
\delta=\frac{\varepsilon}{2 m \max _{i=1, \ldots, m}\left|c_{i}\right|}
$$


then $x-y \in U\left(\chi_{1}, \ldots, \chi_{m} ; \delta\right)$ implies $\sum_{i=1}^{m}\left|c_{i}\right| \cdot\left|\chi_{i}(x)-\chi_{i}(y)\right|<\frac{\varepsilon}{2}$ and so also $\left|\sum_{i=1}^{m} c_{i} \chi_{i}(x)-\sum_{i=1}^{m} c_{i} \chi_{i}(y)\right|<\frac{\varepsilon}{2}$. Consequently,

$$
|f(x)-f(y)| \leq\left|f(x)-\sum_{i=1}^{m} c_{i} \chi_{i}(x)\right|+\left|\sum_{i=1}^{m} c_{i} \chi_{i}(x)-\sum_{i=1}^{m} c_{i} \chi_{i}(y)\right|+\left|\sum_{i=1}^{m} c_{i} \chi_{i}(y)-f(y)\right|<\varepsilon .
$$

(b) $\Rightarrow$ (a) Let $\beta X$ be the Cech-Stone compactification of $X$ endowed with the discrete topology. If $F: X \rightarrow \mathbb{C}$ is bounded, there exists a unique continuous extension $F^{\beta}$ of $F$ to $\beta X$. Let $\mathcal{S}$ be the collection of all continuous functions $g$ on $\beta X$ such that $g=\sum_{j=1}^{n} c_{j} \chi_{j}^{\beta}$ with $\chi_{j} \in H, c_{j} \in \mathbb{C}$ and $n \in \mathbb{N}_{+}$. Then $\mathcal{S}$ is a subalgebra of $\mathcal{C}(\beta X, \mathbb{C})$ closed under conjugation and contains all constants. In fact in $\mathcal{S}$ we have $\chi_{k}^{\beta} \chi_{j}^{\beta}=\left(\chi_{k} \chi_{j}\right)^{\beta}$ by definition and $\overline{\chi^{\beta}}=(\bar{\chi})^{\beta}$ because $\chi \bar{\chi}=1$ and so $(\chi \bar{\chi})^{\beta}=\chi^{\beta}(\bar{\chi})^{\beta}=1$, that is $(\bar{\chi})^{\beta}=\left(\chi^{-1}\right)^{\beta}=\overline{\chi^{\beta}}$.

Now we will see that $\mathcal{S}$ separates the points of $\beta X$ separated by $f^{\beta}$, to apply the local Stone-Weierstraß Theorem 2.19. Let $x, y \in \beta X$ and $f^{\beta}(x) \neq f^{\beta}(y)$. Consider two nets $\left\{x_{i}\right\}_{i}$ and $\left\{y_{i}\right\}_{i}$ in $X$ such that $x_{i} \rightarrow x$ and $y_{i} \rightarrow y$. Since $f^{\beta}$ is continuous, we have $f^{\beta}(x)=\lim f\left(x_{i}\right)$ and $f^{\beta}(y)=\lim f\left(y_{i}\right)$. Along with $f^{\beta}(x) \neq f^{\beta}(y)$ this implies that there exists $\varepsilon>0$ such that $\left|f\left(x_{i}\right)-f\left(y_{i}\right)\right| \geq \varepsilon$ for every sufficiently large $i$. By the hypothesis there exist $\delta>0$ and $\chi_{1}, \ldots, \chi_{k} \in H$ such that for every $u, v \in X$ if $u-v \in U_{G}\left(\chi_{1}, \ldots, \chi_{k} ; \delta\right)$ then $|f(u)-f(v)|<\varepsilon$. Assume $\chi_{j}^{\beta}(x)=\chi_{j}^{\beta}(y)$ holds true for every $j=1, \ldots, k$. Then $x_{i}-y_{i} \in U_{G}\left(\chi_{1}, \ldots, \chi_{k} ; \delta\right)$ for every sufficiently large $i$, this contradicts (a). So each pair of points of $\beta X$ separated by $f^{\beta}$ is also separated by $\mathcal{S}$. Since $\beta X$ is compact, one can apply the local version of the Stone-Weierstraß Theorem 2.19 to $\mathcal{S}$ and $f^{\beta}$ and so $f^{\beta}$ can be uniformly approximated by $\mathcal{S}$. To conclude note that if $g=\sum c_{j} \chi_{j}^{\beta}$ on $\beta X$ then $g\left\lceil X=\sum c_{j} \chi_{j}\right.$.

The reader familiar with uniform spaces will note that item (b) is nothing else but uniform continuity of $f$ w.r.t. the uniformity on $X$ induced by the uniformity of the whole group $G$ determined by the topology $\mathcal{T}_{H}$.

Theorem 5.12 (Følner theorem). Let $G$ be a topological abelian group. If $k$ is a positive integer and $E$ is a subset of $G$ such that $k$ translates of $E$ cover $G$, then for every neighborhood $U$ of 0 in $G$ there exist $\chi_{1}, \ldots, \chi_{m} \in \widehat{G}$, where $m=k^{2}$, and $\delta>0$ such that $U_{G}\left(\chi_{1}, \ldots, \chi_{m} ; \delta\right) \subseteq U-U+E_{(8)}$.

Proof. By Følnerś lemma 5.7 there exist $\varphi_{1}, \ldots, \varphi_{m} \in G^{*}$ such that $U_{G}\left(\varphi_{1}, \ldots, \varphi_{m} ; \frac{\pi}{2}\right) \subseteq E_{(8)}$, where the characters $\varphi_{j}$ can be discontinuous. Our aim will be to replace these characters by continuous ones "enlarging" $E_{(8)}$ to $U-U+E_{(8)}$.

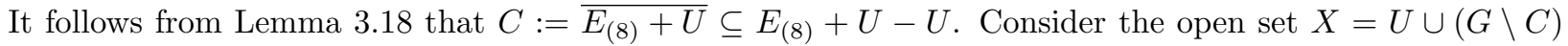
and the function $f: X \rightarrow \mathbb{C}$ defined by

$$
f(x)= \begin{cases}0 & \text { if } x \in U \\ 1 & \text { if } x \in G \backslash C\end{cases}
$$

Then $f$ is continuous as $X=U \cup(G \backslash C)$ is a clopen partition of $X$.

Let $H$ be the group generated by $\varphi_{1}, \ldots, \varphi_{m}$. Take $x, y \in X$ with $x-y \in U_{G}\left(\varphi_{1}, \ldots, \varphi_{m} ; \frac{\pi}{2}\right) \subseteq E_{(8)}$. So if $y \in U$ then $x \in E_{(8)}+U$ and consequently $x \notin G \backslash \overline{E_{(8)}+U}$, that is $x \in U$. In the same way it can be showed that $x \in U$ yields $y \in U$. This gives $f(x)=f(y)$ by the definition of $f$. So by Proposition 5.11 one can uniformly approximate $f$ on $X$ by characters of $H$. Hence one can find a finite number of $m$-uples $\tilde{j}=\left(j_{1}, \ldots, j_{m}\right)$ of integers and $c_{\tilde{j}} \in \mathbb{C}$ such that

$$
\left|\sum_{\tilde{j}} c_{\tilde{j}} \varphi_{1}^{j_{1}}(x) \cdot \ldots \cdot \varphi_{m}^{j_{m}}(x)-f(x)\right| \leq \frac{1}{3}
$$

holds for every $x \in X$. Since $X$ is open and $f$ is continuous, we can apply Lemma 5.10 to the convex closed set $M=\left\{z \in \mathbb{C}:|z| \leq \frac{1}{3}\right\}$ and this permits us to assume that all products $\varphi_{1}^{j_{1}} \ldots \cdot \varphi_{m}^{j_{m}}$ are continuous. Letting $x=0$ in (13) one gets $\left|\sum_{\tilde{j}} c_{\tilde{j}}\right| \leq \frac{1}{3}$, and consequently,

$$
\frac{2}{3} \leq\left|\sum_{\tilde{j}} c_{\tilde{j}}-1\right|
$$

Let now $\Phi$ be the subgroup of $H$ consisting of all continuous characters of $H$, i.e., $\Phi=H \cap \widehat{G}$. By Theorem 2.1 there exist $\chi_{1}, \ldots, \chi_{m} \in \Phi$ that generate $\Phi$. Choose $\varepsilon>0$ with $\varepsilon \sum_{\tilde{j}}\left|c_{\tilde{j}}\right|<\frac{1}{3}$. By the continuity of $\chi_{1}, \ldots, \chi_{m} \in \Phi$ there exists $\delta>0$ such that $x \in U_{G}\left(\chi_{1}, \ldots, \chi_{m} ; \delta\right)$ implies $\left|\varphi_{1}^{j_{1}}(x) \cdot \ldots \cdot \varphi_{m}^{j_{m}}(x)-1\right| \leq \varepsilon$ for all summands $\varphi_{1}^{j_{1}} \cdot \ldots \cdot \varphi_{m}^{j_{m}}$ in $(13)$. 
To prove

$$
U_{G}\left(\chi_{1}, \ldots, \chi_{m} ; \delta\right) \subseteq U-U+E_{(8)}
$$

assume for a contradiction that some $z \in U_{G}\left(\chi_{1}, \ldots, \chi_{m} ; \delta\right)$ and $z \notin U-U+E_{(8)}$. Since $C=\overline{E_{(8)}+U} \subseteq$ $E_{(8)}+U-U$, then $z \in G \backslash C \subseteq X$. Thus, by the definition of $f$, (13), (14) and $\left|\varphi_{1}^{j_{1}}(z) \cdot \ldots \cdot \varphi_{m}^{j_{m}}(z)-1\right| \leq \varepsilon$, we have

$$
\frac{2}{3} \leq\left|\sum_{\tilde{j}} c_{\tilde{j}}-1\right| \leq\left|\sum_{\tilde{j}} c_{\tilde{j}}\left(1-\varphi_{1}^{j_{1}}(z) \cdot \ldots \cdot \varphi_{m}^{j_{m}}(z)\right)\right|+\left|\sum_{\tilde{j}} c_{\tilde{j}} \varphi_{1}^{j_{1}}(z) \cdot \ldots \cdot \varphi_{m}^{j_{m}}(z)-f(z)\right| \leq \varepsilon \sum_{\tilde{j}}\left|c_{\tilde{j}}\right|+\frac{1}{3} .
$$

These inequalities together give $\frac{2}{3} \leq \varepsilon \sum_{\tilde{j}}\left|c_{\tilde{j}}\right|+\frac{1}{3}$. This contradicts the choice of $\varepsilon$.

\section{Peter-Weyl's theorem and other applications of Følner's theorem}

In this section we prove Peter-Weyl's theorem using Følner's theorem.

\subsection{Precompact group topologies on abelian groups}

Let us recall here that for an abelian group $G$ and a subgroup $H$ of $G^{*}$, the group topology $\mathcal{T}_{H}$ generated by $H$ is the coarsest group topology on $G$ that makes every character from $H$ continuous. We recall its description and properties in the next proposition:

Proposition 6.1. Let $G$ be an abelian group and let $H$ be a group of characters of $G$. A base of the neighborhoods of 0 in $\left(G, \mathcal{T}_{H}\right)$ is given by the sets $U\left(\chi_{1}, \ldots, \chi_{m} ; \delta\right)$, where $\chi_{1}, \ldots, \chi_{m} \in H$ and $\delta>0$. Moreover $\left(G, \mathcal{T}_{H}\right)$ is a Hausdorff if and only if $H$ separates the points of $G$.

Now we can characterize the precompact topologies on abelian groups.

Theorem 6.2. Let $(G, \tau)$ be an abelian group. The following conditions are equivalent:

(a) $\tau$ is precompact;

(b) $\tau$ is Hausdorff on $G$ and the neighborhoods of 0 in $G$ are big subsets;

(c) there exists a group $H$ of continuous characters of $G$ that separates the points of $G$ and such that $\tau=\mathcal{T}_{H}$.

Proof. (a) $\Rightarrow(\mathrm{b})$ is the definition of precompact topology.

(b) $\Rightarrow$ (c) If $H=\widehat{(G, \tau)}$ then $\mathcal{T}_{H} \subseteq \tau$. Let $U$ and $V$ be open neighborhoods of 0 in $(G, \tau)$ such that $V_{(10)} \subseteq U$. Then $V$ is big and by Følner's Theorem 5.12 there exist continuous characters $\chi_{1}, \ldots, \chi_{m}$ of $G$ such that $U_{G}\left(\chi_{1}, \ldots, \chi_{m} ; \delta\right) \subseteq V_{(10)} \subseteq U$ for some $\delta>0$. Thus $U \in \mathcal{T}_{H}$ and $\tau \subseteq \mathcal{T}_{H}$.

(c) $\Rightarrow$ (a) Even if this implication is contained in Corollary 4.31, we give a direct proof here. Let $i: G \rightarrow \mathbb{S}^{H}$ be defined by $i(g)=i_{g}: H \rightarrow \mathbb{S}$ (if $g \in G$ ) with $i_{g}(\chi)=\chi(g)$ for every $\chi \in H$. Since $H$ separates the points of $G$, the function $i$ is injective. The product $\mathbb{S}^{H}$ endowed with the product topology is compact and so $i$ is a topological immersion by Proposition 6.1. The closure of $i(G)$ in $\mathbb{S}^{H}$ is compact and $\widetilde{G}$ is isomorphic to it, hence $\widetilde{G}$ is compact.

Remark 6.3. The above theorem essentially belongs to Comfort and Ross [23]. It can be given in the following simpler "Hausdorff-free" version: $\tau$ is totally bounded iff $\tau=\mathcal{T}_{H}$ for some group $H$ of continuous characters of $G$.

Corollary 6.4 (Peter-Weyl's theorem). If $G$ is a compact abelian group, then $\widehat{G}$ separates the points of $G$.

Proof. Let $\tau$ be the topology of $G$. By Theorem 6.2 there exists a group $H$ of continuous characters of $G$ (i.e., $H \subseteq \widehat{G})$ such that $\tau=\mathcal{T}_{H}$. Since $\tau \supseteq \mathcal{T}_{\widehat{G}}$ and $H \subseteq \widehat{G}$ we conclude that $H=\widehat{G}$ separates the points of $G$.

The next theorem will allow us to sharpen this property (see Corollary 6.6).

Theorem 6.5. Let $G$ be an abelian group. Let $\mathcal{H}$ be the set of all groups of characters of $G$ separating the points of $G$ and $\mathcal{P}$ be the set of all precompact group topologies on $G$. Then the function $T: \mathcal{H} \rightarrow \mathcal{P}$ which associates to $H \in \mathcal{H}$ the topology $\mathcal{T}_{H} \in \mathcal{P}$ is an order preserving bijection (if $H_{1}, H_{2} \in \mathcal{H}$ then $\mathcal{T}_{H_{1}} \subseteq \mathcal{T}_{H_{2}}$ if and only if $H_{1} \subseteq H_{2}$ ). 
Proof. The equivalence $(\mathrm{a}) \Leftrightarrow(\mathrm{c})$ of Theorem 6.2 yields that $\mathcal{T}_{H} \in \mathcal{P}$ for every $H \in \mathcal{H}$ and that $T$ is surjective.

Let $H \in \mathcal{H}$ and suppose that $\chi \in\left(\widehat{G, \mathcal{T}_{H}}\right)$. To show that $\chi \in H$ let $\varepsilon>0$. Since $\chi$ is continuous in 0 , by Proposition 6.1 there exist $\chi_{1}, \ldots, \chi_{m} \in H$ and $\delta>0$ such that $|\chi(x)-1|<\varepsilon$ for $x \in U\left(\chi_{1}, \ldots, \chi_{m} ; \delta\right)$. Therefore for every $x, y \in G$ with $x-y \in U\left(\chi_{1}, \ldots, \chi_{m} ; \delta\right)$ we get $|\chi(x-y)-1|<\varepsilon$, that is $|\chi(x)-\chi(y)|<\varepsilon$. Apply now Proposition 5.11 to find $\chi_{1}, \ldots, \chi_{m} \in H$ and $c_{i}, \ldots, c_{m} \in \mathbb{C}$ such that $\left|\sum_{j=1}^{m} c_{j} \chi_{j}(x)-\chi(x)\right| \leq \frac{1}{2}$ for every $x \in G$. This yields $\left|\sum_{j=1}^{m} c_{j} \chi_{j}(x) \chi^{-1}(x)-1\right| \leq \frac{1}{2}$.

Suppose now that $\chi \notin H$. Then each $\chi_{j} \chi^{-1}$ in the previous condition is non-constant. Equip $G$ with the indiscrete topology. Then each character $\chi_{j} \chi^{-1}$ is discontinuous. Applying Lemma 5.10 we get the inequality $1 \leq \frac{1}{2}$, which is a contradiction. Therefore $\chi \in H$ and so $H=\left(\widehat{G, \tau_{H}}\right)$ for every $H \in \mathcal{H}$.

If $H_{1}, H_{2} \in \mathcal{H}$ and $\mathcal{T}_{H_{1}}=\mathcal{T}_{H_{2}}$ then $H_{1}=H_{2}$, so $T$ is a bijection.

The last statement of the theorem is obvious.

As a corollary of Theorem 6.5 we obtain the following important fact that completes Corollary 6.4. It will be essentially used in the proof of the duality theorem.

Corollary 6.6. If $(G, \tau)$ is a compact abelian group and $H \leq \widehat{G}$ separates the points of $G$, then $H=\widehat{G}$.

Proof. By Theorem 6.2 it holds $\tau=\mathcal{T}_{\widehat{G}}$. Since $\mathcal{T}_{H} \subseteq \mathcal{T}_{\widehat{G}}$ by Theorem 6.5 and $\mathcal{T}_{H}$ is Hausdorff, then $\mathcal{T}_{H}=\mathcal{T}_{\widehat{G}}$. Now again Theorem 6.5 yields $H=\widehat{G}$.

Definition 6.7. An abelian topological group is elementary compact if it is topologically isomorphic to $\mathbb{T}^{s} \times F$, where $n$ is a positive integer and $F$ is a finite abelian group.

Proposition 6.8. Let $G$ be a compact abelian group and let $U$ be an open neighborhood of 0 in $G$. Then there exists a closed subgroup $C$ of $G$ such that $C \subseteq U$ and $G / C$ is an elementary compact abelian group. In particular, $G$ is an inverse limit of elementary compact abelian groups.

Proof. By the Peter-Weyl Theorem 6.4 $\bigcap_{\chi \in \widehat{G}}$ ker $\chi=\{0\}$ and each ker $\chi$ is a closed subgroup of $G$. By the compactness of $G$ there exists a finite subset $F$ of $\widehat{G}$ such that $C=\bigcap_{\chi \in F}$ ker $\chi \subseteq U$. Define now $g=\prod_{\chi \in F} \chi$ : $G \rightarrow \mathbb{T}^{F}$. Thus ker $g=C$ and $G / C$ is topologically isomorphic to the closed subgroup $g(G)$ of $\mathbb{T}^{F}$ by the compactness of $G$. So $G / C$ is elementary compact abelian by Lemma 4.47 .

To prove the last statement, fix for every open neighborhood $U_{i}$ of 0 in $G$ a closed subgroup $C_{i}$ of $G$ with $C_{i} \subseteq U$ and such that $G / C_{i}$ is elementary compact abelian. Note that for $C_{i}$ and $C_{j}$ obtained in this way the subgroup $C_{i} \cap C_{j}$ has the same property as $G / C_{i} \cap C_{j}$ is isomorphic to a closed subgroup of the product $G / C_{i} \times G / C_{j}$ which is again an elementary compact abelian group. Enlarging the family $\left(C_{i}\right)$ with all finite intersections we obtain an inverse system of elementary compact abelian groups $G / C_{i}$ where the connecting homomorphisms $G / C_{i} \rightarrow G / C_{j}$, when $C_{i} \leq C_{j}$, are simply the induced homomorphisms. Then the inverse limit $G^{\prime}$ of this inverse system is a compact abelian group together with a continuous homomorphism $f: G \rightarrow G^{\prime}$ induced by the projections $p_{i}: G \rightarrow G / C_{i}$. Assume $x \in G$ is non-zero. Pick on open neighborhood $U$ of 0 . By the first part of the proof, there exists $C_{i} \subseteq U$, hence $x \notin C_{i}$. Therefore, $p_{i}(x) \neq 0$, so $f(x) \neq 0$ as well. This proves that $f$ is injective. To check surjectivity of $f$ take an element $x^{\prime}=\left(x_{i}+C_{i}\right)$ of the inverse limit $G^{\prime}$. Then the family of closed cosets $x_{i}+C_{i}$ in $G$ has the finite intersection property, so has a non-empty intersection. For every element $x$ of that intersection one has $f(x)=x^{\prime}$. Finally, the continuous isomorphism $f: G \rightarrow G{ }^{\prime}$ must be open by the compactness of $G$.

For a topological abelian group $G$ we say that $G$ has no small subgroups, or shortly, $G$ is $N S S$, if there exists a neighborhood $U$ of 0 such that $U$ contain no non-trivial subgroups of $G$. It follows immediately from the above proposition that the compact abelian group $G$ has no small subgroups precisely when $G$ is an elementary compact abelian group.

\subsection{Precompact group topologies determined by sequences}

Large and lacunary sets (mainly in $\mathbb{Z}$ or elsewhere) are largely studied in number theory, harmonic analysis and dynamical systems ([43], [24], [80], [52], [53], [54], [55], [59]).

Let us consider a specific problem. For a strictly increasing sequence $\underline{u}=\left(u_{n}\right)_{n \geq 1}$ of integers, the interest in the distribution of the multiples $\left\{u_{n} \alpha: n \in \mathbb{N}\right\}$ of a non-torsion element $\alpha$ of the group $\mathbb{T}=\mathbb{R} / \mathbb{Z}$ has roots in number theory (Weyl's theorem of uniform distribution modulo 1) and in ergodic theory (Sturmian sequences and Hartman sets [99]). According to Weyl's theorem, the set $\left\{u_{n} \alpha: n \in \mathbb{N}\right\}$ will be uniformly dense in $\mathbb{T}$ for almost all $\alpha \in \mathbb{T}$. One can consider the subset $t_{\underline{u}}(\mathbb{T})$ of all elements $\alpha \in \mathbb{T}$ such that $\lim _{n} u_{n} \alpha=0$ in $\mathbb{T}$. Clearly 
it will have measure zero. Moreover, it is a subgroup of $\mathbb{T}$ as well as a Borel set, so it is either countable or has size $\mathfrak{c}$. It was observed by Armacost [3] that when $u_{n}=p^{n}$ for all $n$ and some prime $p$, then $t_{\underline{u}}(\mathbb{T})=\mathbb{Z}\left(p^{\infty}\right)$. He posed the question of describing the subgroup $t_{u}(\mathbb{T})$ for the sequence $u_{n}=n$ !, this was done by Borel [19] (see also [36] and [31] for the more general problem concerning sequences $\underline{u}$ with $u_{n-1} \mid u_{n}$ for every $n$ ).

Another motivation for the study of the subgroups of the form $t_{\underline{u}}(\mathbb{T})$ come from the fact that they lead to the description of precompact group topologies on $\mathbb{Z}$ that make the sequence $u_{n}$ converge to 0 in $\mathbb{Z}$ (see the comment after proposition 6.9). Let us start by an easy to prove general fact:

Proposition 6.9. [7] A sequence $A=\left\{a_{n}\right\}_{n}$ in a precompact abelian group $G$ converges to 0 in $G$ iff $\chi\left(a_{n}\right) \rightarrow 0$ in $\mathbb{T}$ for every continuous character of $G$.

In the case of $G=\mathbb{Z}$ the characters of $G$ are simply simply elements of $\mathbb{T}$, i.e., a precompact group topology on $\mathbb{Z}$ has the form $\mathcal{T}_{H}$ for some subgroup $H$ of $\mathbb{T}$. Thus the above proprosition for $G=\mathbb{Z}$ can be reformulated as: a sequence $A=\left\{a_{n}\right\}_{n}$ in $\left(\mathbb{Z}, \mathcal{T}_{H}\right)$ converges to 0 iff $a_{n} x \rightarrow 0$ for every $x \in H$, i.e., simply $H \subseteq t_{\underline{a}}(\mathbb{T})$.

Now we can discuss a counterpart of the notion of $T$-sequences (introduced in $\S 3.5$ ), defined with respect to topologies induced by characters, i.e., precompact topologies.

Definition 6.10. $[7,9]$ A sequence $A=\left\{a_{n}\right\}_{n}$ in an abelian group $G$ is called a TB-sequence is there exists a precompct group topology on $G$ such that $a_{n} \rightarrow 0$.

Clearly, every TB-sequence is a T-sequence (see Example 6.12 for a T-sequence in $\mathbb{Z}$ that is not a TBsequence). The advantage of TB-sequences over the T-sequences is in the easier way of determining sufficient condition for a sequence to be a TB-sequence [7, 9]. For example, a a sequence $\left(a_{n}\right)$ in $\mathbb{Z}$ is a TB-sequence iff the subgroup $t_{a}(\mathbb{T})$ of $\mathbb{T}$ is infinite.

Egglestone [42] proved that the asymptotic behavior of the sequence of ratios $q_{n}=\frac{u_{n+1}}{u_{n}}$ may have an impact on the size of the subgroup $t_{\underline{u}}(\mathbb{T})$ in the following remarkable dichotomy:

Theorem 6.11. Let $\left(a_{n}\right)$ be a sequence in $\mathbb{Z}$.

- If $\lim _{n} \frac{a_{n+1}}{a_{n}}=+\infty$, then $\left(a_{n}\right)$ is a TB-sequence and $\left|t_{\underline{a}}(\mathbb{T})\right|=\mathfrak{c}$.

- If $\frac{a_{n+1}}{a_{n}}$ is bounded, then $t_{\underline{a}}(\mathbb{T})$ is countable.

Example 6.12. [9] There exists a TB-sequence $\left(a_{n}\right)$ in $\mathbb{Z}$ with $\lim _{n} \frac{a_{n+1}}{a_{n}}=1$.

Here is an example of a $\mathrm{T}$-sequence in $\mathbb{Z}$ that is not a TB-sequence.

Example 6.13. For every $T B$-sequence $A=\left\{a_{n}\right\}$ in $\mathbb{Z}$ such that $t_{\underline{a}}(\mathbb{T})$ is countable, there exists a sequence $\left\{c_{n}\right\}$ in $\mathbb{Z}$ such that the sequence $q_{n}$ defined by $q_{2 n}=c_{n}$ and $q_{2 n-1}=a_{n}$, is a $T$-sequence, but not a $T B$-sequence.

Proof. Let $\left\{z_{1}, \ldots, z_{n}, \ldots\right\}$ be an enumeration of $t_{\underline{a}}(\mathbb{T})$.

According to Lemma 3.51 there exists a sequence $b_{n}$ in $\mathbb{Z}$ such that for every choice of the sequence $\left(e_{n}\right)$, where $e_{n} \in\{0,1\}$, the sequence $q_{n}$ defined by $q_{2 n}=b_{n}+e_{n}$ and $q_{2 n-1}=a_{n}$, is a $T$-sequence. Now we define the sequence $q_{n}$ with $q_{2 m-1}=a_{m}$ and $q_{2 m}=b_{m}$ when $m$ is not a prime power. Let $p_{1}, \ldots, p_{n}, \ldots$ be all prime numbers enumerated one-to-one. Now fix $k$ and define $e_{k} \in\{0,1\}$ depending on $\lim _{n} b_{p_{k}^{n}} z_{k}$ as follows:

- if $\lim _{n} b_{p_{k}^{n}} z_{k}=0$, let $e_{k}=1$,

- if $\lim _{n} b_{p_{k}^{n}} z_{k} \neq 0$ (in particular, if the limit does not exists) let $e_{k}=0$.

Now let $q_{2 p_{k}^{n}}=b_{p_{k}^{n}}+e_{k}$ for $n \in \mathbb{N}$. Hence for every $k \in \mathbb{N}$

$$
\lim _{n} q_{2 p_{k}^{n}} z_{k}=0 \Longrightarrow e_{k}=1
$$

To see that $\left(q_{n}\right)$ is not a $T B$-sequence assume that $\chi: \mathbb{Z} \rightarrow \mathbb{T}$ a character such that $\chi\left(q_{n}\right) \rightarrow 0$ in $\mathbb{T}$. Then $x=\chi(1) \in \mathbb{T}$ satisfies $q_{n} x \rightarrow 0$, so $x \in t_{\underline{q}}(\mathbb{T}) \subseteq t_{\underline{a}}(\mathbb{T})$. So there exists $k \in \mathbb{N}$ with $x=z_{k}$. By $(*) e_{k}=1$. Hence $q_{2 p_{k}^{n}}=b_{p_{k}^{n}}+1$ and $\lim _{n} b_{p_{k}^{n}} z_{k}=0$, so $\bar{x} \in t_{\underline{q}}(\mathbb{T})$ yields $0=\lim _{n} q_{2 p_{k}^{n}} x=0+x$, i.e., $x=0$. This proves that every character $\chi: \mathbb{Z} \rightarrow \mathbb{T}$ such that $\chi\left(q_{n}\right) \rightarrow 0$ in $\mathbb{T}$ is trivial. In particular, $\left(q_{n}\right)$ not a $T B$-sequence.

Let us note that the above proof gives much more. Since $q_{n} \rightarrow 0$ in $\tau_{\left(q_{n}\right)}$, it shows that every $\tau_{\left(q_{n}\right)}$-continuous character of $\mathbb{Z}$ is trivial, i.e., $\left(\widehat{\mathbb{Z}, \tau_{\left(q_{n}\right)}}=0\right.$.

The information accumulated on the properties of the subgroups $t_{\underline{u}}(\mathbb{T})$ of $\mathbb{T}$ motivated the problem of describing those subgroups $H$ of $\mathbb{T}$ that can be characterized as $H=t_{\underline{u}}(\mathbb{T})$ for some sequence $\underline{u}$. As already 
mentioned, such an $H$ can be only countable or can have size $\mathfrak{c}$ being of measure zero. A measure zero subgroup $H$ of $\mathbb{T}$ of size $\mathfrak{c}$ that is not even contained in any proper subgroup of $\mathbb{T}$ of the form $t_{u}(\mathbb{T})$ was built in $[7]$ (under the assumption of Martin Axiom) and in later in [61, 62] (in ZFC). Much earlier Borel [19] had already resolved in the positive the remaining part of the problem showing that every countable subgroup of $\mathbb{T}$ can be characterized (in the above sense). Unaware of his result, Larcher [74], and later Kraaikamp and Liardet [71], proved that some cyclic subgroups of $\mathbb{T}$ are characterizable (see also $[16,15,12,14,13]$ for related results). The paper [9] describes the algebraic structure of the subgroup $t_{\underline{u}}(\mathbb{T})$ when the sequence $\underline{u}:=\left(u_{n}\right)$ verifies a linear recurrence relation of order $\leq k$,

$$
u_{n}=a_{n}^{(1)} u_{n-1}+a_{n}^{(2)} u_{n-2}+\ldots+a_{n}^{(k)} u_{n-k}
$$

for every $n>k$ with $a_{n}^{(i)} \in \mathbb{Z}$ for $i=1, \ldots, k$.

Three proofs of Borel's theorem of characterizability of the countable subgroups of $\mathbb{T}$ were given in [13]. These author mentioned that the theorem can be extended to compact abelian groups in place of $\mathbb{T}$, without giving any precise formulation. There is a natural way to extend the definition of $t_{\underline{u}}(\mathbb{T})$ to an arbitrary topological abelian group $G$ by letting $t_{\underline{u}}(G)=\left\{x \in G: \lim _{n} u_{n} x=0\right.$ in $\left.G\right\}$. Actually, for the sequence $u_{n}=p^{n}$ (resp., $u_{n}=n$ !) an element $x$ satisfying $\lim _{n} u_{n} x=0$ has been called topologically p-torsion (resp., topologically torsion) by Braconnier and Vilenkin in the forties of the last century and these notions played a prominent role in the development of the theory of locally compact abelian groups. One can easily reduce the computation of $t_{\underline{u}}(G)$ for an arbitrary locally compact abelian group to that of $t_{\underline{u}}(\mathbb{T})[26]$. Independently on their relevance in other questions, the subgroups $t_{u}(G)$ turned out to be of no help in the characterization of countable subgroups of the compact abelian groups. Indeed, a much weaker condition, turned out the characterize the circle group $\mathbb{T}$ in the class of all locally compact abelian groups:

Theorem 6.14. [31] In a locally compact abelian group $G$ every cyclic subgroup of the group $G$ is an intersection of subgroups of the form $t_{\underline{u}}(G)$ iff $G \cong \mathbb{T}$.

Actually, one can remove the "abelian" restraint in the theorem remembering that in the non-abelian case $t_{\underline{u}}(G)$ is just a subset of $G$, not a subgroup in general [31].

The above theorem suggested to use in [35] a different approach to the problem, replacing the sequence of integers $u_{n}$ (characters of $\left.\mathbb{T} !\right)$ by a sequence $u_{n}$ in the Pontryagin-van Kampen dual $\widehat{G}$. Then the subgroup $s_{\underline{u}}(G)=\left\{x \in G: \lim _{n} u_{n}(x)=0\right.$ in $\left.\mathbb{T}\right\}$ of $G$ really can be used for such a characterization of all countable subgroups of the compact metrizable groups (see $[35,33,17]$ for major detail).

\subsection{On the structure of compactly generated locally compact abelian groups}

From now on all groups are Hausdorff; quotients are taken for closed subgroups and so they are still Hausdorff.

An abelian topological group is elementary locally compact if it is topologically isomorphic to $\mathbb{R}^{n} \times \mathbb{Z}^{m} \times \mathbb{T}^{s} \times F$, where $n, m, s$ are positive integers and $F$ is a finite abelian group. Observe that the class of elementary locally compact abelian groups is closed under taking quotient, closed subgroups and finite products (see Theorem 4.37 and Corollary 4.47).

Lemma 6.15. Let $G$ be a locally compact monothetic group. Then $G$ is either compact or is discrete.

Proof. If $G$ is finite, then $G$ is both compact and discrete. So we can suppose without loss of generality that $\langle x\rangle \cong \mathbb{Z}$ is infinite and so also that $\mathbb{Z}$ is a subgroup of $G$.

If $G$ induces the discrete topology on $\mathbb{Z}$, then $\mathbb{Z}$ is closed and so $G=\mathbb{Z}$ is discrete.

Suppose now that $G$ induces on $\mathbb{Z}$ a non-discrete topology. Our aim is to show that it is totally bounded. Then the density of $\mathbb{Z}$ in $G$ yields that $G=\widetilde{\mathbb{Z}}=\overline{\mathbb{Z}}$ is compact, as $G$ is locally compact and so complete (see Lemma 4.7).

Every open subset of $G$ has no maximal element. Indeed, if $U$ is an open subset of $\mathbb{Z}$ that contains 0 and it has a maximal element, then $-U$ is an open subset of $\mathbb{Z}$ that contains 0 and it has a minimal element and $U \cap-U$ is an open finite neighborhood of 0 in $\mathbb{Z}$; thus $\mathbb{Z}$ is discrete against the assumption. Consequently every open subset of $\mathbb{Z}$ contains positive elements.

Let $U$ be a compact neighborhood of 0 in $G$ and $V$ a symmetric neighborhood of 0 in $G$ such that $V+V \subseteq U$. There exist $g_{1}, \ldots, g_{m} \in G$ such that $U \subseteq \bigcup_{i=1}^{m}\left(g_{i}+V\right)$. Let $n_{1}, \ldots, n_{m} \in \mathbb{Z}$ be positive integers such that $n_{i} \in g_{i}+V$ for every $i=1, \ldots, m$. Equivalently $g_{i} \in n_{i}-V=n_{i}+V$. Thus

$$
U \subseteq \bigcup_{i=1}^{m}\left(g_{i}+V\right) \subseteq \bigcup_{i=1}^{m}\left(n_{i}+V+V\right) \subseteq \bigcup_{i=1}^{m}\left(n_{i}+U\right)
$$


implies

$$
U \cap \mathbb{Z} \subseteq \bigcup_{i=1}^{m}\left(n_{i}+U \cap \mathbb{Z}\right) .
$$

We show that $U \cap \mathbb{Z}$ is big with respect to $\mathbb{Z}$. Let $t \in \mathbb{Z}$; since $U \cap \mathbb{Z}$ has no maximal element, then there exists $s \in U \cap \mathbb{Z}$ such that $s \geq t$. Define $s_{t}=\min \{s \in U \cap \mathbb{Z}: s \geq t\}$. By (1) $s_{t}=n_{i}+u_{t}$ for some $i \leq m$ and $u_{t} \in U \cap \mathbb{Z}$. Since $n_{i}>0$, then $u_{t}<s_{t}$ and so $u_{t}<t \leq s_{t}$. Now put $N=\max \left\{n_{1}, \ldots, n_{m}\right\}$ and $F=\{1, \ldots, N\}$. Hence $U \cap \mathbb{Z}+F=\mathbb{Z}$. This proves that the topology induced on $\mathbb{Z}$ by $G$ is totally bounded.

Corollary 6.16. Let $G$ be a locally compact abelian group and $x \in G$. Then $\overline{\langle x\rangle}$ is either compact or discrete.

Proposition 6.17. Let $G$ be a compactly generated locally compact abelian group. Then there exists a discrete subgroup $H$ of $G$ such that $H \cong \mathbb{Z}^{n}$ for some $n \in \mathbb{N}$ and $G / H$ is compact.

Proof. Suppose first that there exist $g_{1}, \ldots, g_{m} \in G$ such that $G=\overline{\left\langle g_{1}, \ldots, g_{m}\right\rangle}$. We proceed by induction. For $m=1$ apply Lemma 6.15: if $G$ is infinite and discrete take $H=G$ and if $G$ is compact $H=\{0\}$. Suppose now that the property holds for $m \geq 1$ and $G=\overline{\left\langle g_{1}, \ldots, g_{m+1}\right\rangle}$. If every $\overline{\left\langle g_{i}\right\rangle}$ is compact, then so is $G$ and $H=\{0\}$. If $\left\langle g_{m+1}\right\rangle$ is discrete, consider the canonical projection $\pi: G \rightarrow G_{1}=G /\left\langle g_{m+1}\right\rangle$. Since $G_{1}$ has a dense subgroup generated by $m$ elements, by the inductive hypothesis there exists a discrete subgroup $H_{1}$ of $G_{1}$ such that $H_{1} \cong \mathbb{Z}^{n}$ and $G_{1} / H_{1}$ is compact. Therefore $H=\pi^{-1}\left(H_{1}\right)$ is a closed countable subgroup of $G$. Thus $H$ is locally compact and countable, hence discrete by Lemma 4.8 .

Since $H$ is finitely generated, it is isomorphic to $H_{2} \times F$, where $H_{2} \cong \mathbb{Z}^{s}$ for some $s \in \mathbb{N}$ and $F$ is a finite abelian group (see Theorem 2.1). Now $G / H$ is isomorphic to $G_{1} / H_{1}$ and $H / H_{2}$ is finite, so $G / H_{2}$ is compact thanks to Lemma 4.5 .

Now consider the general case. There exists a compact subset $K$ of $G$ that generates $G$. By Lemma 4.14 we can assume wlog that $K=\bar{U}$, where $U$ is a symmetric neighborhood of 0 in $G$ with compact closure. We show now that there exists a finite subset $F$ of $G$ such that

$$
K+K \subseteq K+\langle F\rangle
$$

In fact, pick a symmetric neighborhood $V$ of 0 in $G$ such that $V+V \subseteq U$. For the compact set $K$ satisfying $K \subseteq \bigcup_{x \in K}(x+V)$ there exists a finite subset $F$ of $K$ such that $K \subseteq \bigcup_{x \in F}(x+V)=F+V$. Then

$$
K+K \subseteq F+F+V+V \subseteq\langle F\rangle+U \subseteq\langle F\rangle+K .
$$

gives (2). An easy inductive argument shows that $\langle K\rangle=G$ and (2) imply $G=\langle K\rangle \subseteq K+\langle F\rangle$.

Let $G_{1}=\langle F\rangle$. By $G=\langle F\rangle+K$ the quotient $\pi(K)=G / G_{1}$ is compact. By the first part of the proof there exists a discrete subgroup $H$ of the locally compact subgroup $G_{1}$ of $G$, such that $H \cong \mathbb{Z}^{n}$ for some $n \in \mathbb{N}$ and $G_{1} / H$ is compact. Since $G_{1} / H$ is a compact subgroup of $G / H$ such that $(G / H) /\left(G_{1} / H\right) \cong G / G_{1}$ is compact, we conclude that also $G / H$ is compact.

Proposition 6.18. Let $G$ be a compactly generated locally compact abelian group. Then there exists a compact subgroup $K$ of $G$ such that $G / K$ is elementary locally compact abelian.

Proof. By Proposition 6.17 there exists a discrete subgroup $H$ of $G$ such that the quotient $G / H$ is compact. Consider the canonical projection $\pi$ of $G$ onto $G / H$. Let $U$ be a compact symmetric neighborhood of 0 in $G$ such that $(U+U+U) \cap H=\{0\}$. So $\pi(U)$ is a neighborhood of 0 in $G / H$ and applying Lemma 6.8 we find a closed subgroup $L \supseteq H$ of $G$ such that the closed subgroup $L / H$ of $G / H$ satisfies

$$
L / H \subseteq \pi(U) \text { and }(G / H) /(L / H)=G / L \cong \mathbb{T}^{t} \times F,
$$

where $F$ is a finite abelian group and $t \in \mathbb{N}$, i.e., $G / L$ is elementary compact abelian.

The set $K=L \cap U$ is compact being closed in the compact neighborhood $U$. Let us see now that $K$ is a subgroup of $G$. To this end take $x, y \in K$. Then $x-y \in L$ and $\pi(x-y) \in C \subseteq \pi(U)$. Thus $\pi(x-y)=\pi(u)$ for some $u \in U$. As $\pi(x-y-u)=0$ in $G / H$, one has $x-y-u \in(U+U+U) \cap H=\{0\}$. Hence $x-y=u \in L \cap U=K$.

Now take $x \in L$; consequently $\pi(x) \in C \subseteq \pi(U)$ so $\pi(x)=\pi(u)$ for some $u \in U$. Clearly, $u \in L \cap U=K$, hence $\pi(L)=\pi(K)$. Thus $L=K+H$ and $K \cap H=\{0\}$ yields that the canonical projection $l: G \rightarrow G / K$ restricted to $H$ is a continuous isomorphism of $H$ onto $l(H)=l(L)$. Let us see now that $l(H)$ is discrete. The compact set $K$ is contained in the open set $W_{1}=G \backslash(H \backslash\{0\})=G \backslash H \cup\{0\}$ ( $H$ is discrete). By Lemma 4.3 (c) there exists an open neighborhood $V$ of 0 in $G$ such that $K+V \subseteq W_{1}$. This implies that $(K+V) \cap H=\{0\}$ and so $(K+V) \cap(K+H)=K$, that gives $l(V) \cap l(H)=\{0\}$ in $G / K$. Thus

$$
l(L)=l(H) \cong H \cong \mathbb{Z}^{s}
$$


is discrete in $G / K$.

Observe that (4) yields the following isomorphisms:

$$
(G / K) / l(L)=(G / K) /(L / K) \cong G / L \cong \mathbb{T}^{t} \times F
$$

Denote by $\varrho$ the composition $G / K \rightarrow G / L \rightarrow \mathbb{T}^{t} \times F$. Let $W$ be a compact neighborhood of 0 in $G / K$ such that $W+W \subseteq l(V)$ and $\varrho(W) \subseteq \mathbb{T}^{t} \times\{0\}$. Then $\varrho \uparrow W$ is injective because $l(V) \cap l(L)=\{0\}$. In particular, $\varrho$ is a local homeomorphism.

Consider now the canonical projection $q: \mathbb{R}^{t} \rightarrow \mathbb{T}^{t}$. Our aim is to lift it to a continuous homomorphism $f: \mathbb{R}^{t} \rightarrow G / K$ such that $\varrho \circ f=q$. The existence of such a lifting is immediate from the facts that both $q$ and $\varrho$ are covering homomorphisms and $\mathbb{R}^{t}$ simply connected. In particular, $D=f\left(\mathbb{R}^{t}\right)$ is an open subgroup of $G / K$ as has a non-empty interior (as $q$ and $\varrho$ are local homeomorphisms). Since $\mathbb{R}^{t}$ is divisible, by Lemma 2.8 $G / K=D \times B$ where $B$ is a discrete subgroup of $G / K$ because $D \cap B=\{0\}$ and $D$ is open. Moreover $B$ is compactly generated as it is a quotient of $G$. Since it is also discrete, $B$ is finitely generated. Then $f: \mathbb{R}^{t} \rightarrow D$ is open by Theorem 4.9 and so $D$ is isomorphic to a quotient of $\mathbb{R}^{t}$, which is elementary locally compact abelian.

For the reader who is not familiar with covering maps we provide now a self-contained proof.

Take an open neighborhood $U$ of 0 in $\mathbb{R}^{t}$ such that $U-U \cap \operatorname{ker} q=\{0\}$ and let $U_{0}=U \cap q^{-1}(\varrho(W))$. Then $U_{0}$ is an open neighborhood of 0 in $\mathbb{R}^{t}$ such that $q \uparrow_{U_{0}}$ is one-to-one from $U_{0}$ to $\varrho(W)$. Pick a symmetric neighborhood $U_{1}$ of 0 in $\mathbb{R}^{t}$ such that $U_{1}+U_{1} \subseteq U_{0}$. Define a map $f: \mathbb{R}^{t} \rightarrow G / K$ as follows: $f\left\lceil_{U_{0}}\right.$ is simply the composition $\varrho^{-1} \circ q$. So $f$ maps $U_{0}$ onto the open subset $\varrho^{-1}\left(q\left(U_{0}\right)\right)$ of $G / K$. If $x \in \mathbb{R}^{t}$ there exists $n \in \mathbb{N}_{+}$ such that $\frac{1}{n} x \in U_{0}$. We put $f(x)=n f\left(\frac{1}{n} x\right)$ and we note that this definition does not depend on $n$. Moreover, $f\left(x_{1}+x_{2}\right)=f\left(x_{1}\right)+f\left(x_{2}\right)$ for every $x_{1}, x_{2} \in U_{1}$.

We can prove now that $f$ is a homomorphism. First of all we note that for every $x \in \mathbb{R}^{t} f \Gamma_{\langle x\rangle}$ is a homomorphism, i.e., $f(k x)=k f(x)$ for every $k \in \mathbb{Z}$. Now take $x, y \in \mathbb{R}^{t}$. There exists an integer $n>0$ such that $\frac{1}{n} x, \frac{1}{n} y \in U_{1}$ and so $\frac{1}{n} x+\frac{1}{n} y \in U_{0}$. By the the previous step

$$
f(x+y)=n f\left(\frac{1}{n}(x+y)\right)=n f\left(\frac{1}{n} x+\frac{1}{n} y\right)=n f\left(\frac{1}{n} x\right)+n f\left(\frac{1}{n} y\right)=f(x)+f(y),
$$

for all $x, y \in \mathbb{R}^{t}$.

So $f$ is continuous and also a local homeomorphism on $\mathbb{R}^{t}$ because it is the composition of local homeomorphisms: restricted to the open subset $U_{0}, f$ is the composition of $q$ and $\varrho^{-1}$ (note that both $\varrho\left\lceil_{W}\right.$ and $q \Upsilon_{U_{0}}$ are continuous and open).

To prove the Pontryagin-van Kampen duality theorem in the general case (for $G \in \mathcal{L}$ ), we need Theorem 6.19, which generalizes the Peter-Weyl Theorem 6.4.

Theorem 6.19. If $G$ is a locally compact abelian group, then $\widehat{G}$ separates the points of $G$.

Proof. Let $V$ be a compact neighborhood of 0 in $G$. Take $x \in G \backslash\{0\}$. Then $G_{1}=\langle V \cup\{x\}\rangle$ is an open (it has non-void interior) compactly generated subgroup of $G$. In particular $G_{1}$ is locally compact. By Proposition 6.17 there exists a discrete subgroup $H$ of $G_{1}$ such that $H \cong \mathbb{Z}^{m}$ for some $m \in \mathbb{N}$ and $G_{1} / H$ is compact. Thus $\bigcap_{n \in \mathbb{N}_{+}} n H=\{0\}$ and so there exists $n \in \mathbb{N}_{+}$such that $x \notin n H$. Since $H / n H$ is finite, the quotient $G_{2}=G_{1} / n H$ is compact by Lemma 4.5. Consider the canonical projection $\pi: G_{1} \rightarrow G_{2}$ and note that $\pi(x)=y \neq 0$ in $G_{2}$. By the Peter-Weyl Theorem 6.4 there exists $\xi \in \widehat{G}$ such that $\xi(y) \neq 0$. Consequently $\chi=\xi \circ \pi \in \widehat{G_{1}}$ and $\chi(x) \neq 0$. By Theorem 2.5 there exists $\bar{\chi} \in \widehat{G}$ such that $\bar{\chi} \uparrow_{G_{1}}=\chi$.

It follows from Theorem 6.19 and Remark 7.24 that $\omega_{G}$ is a continuous monomorphism for every locally compact abelian group $G$.

Corollary 6.20. Let $G$ be a locally compact abelian group and $K$ a compact subgroup of $G$. Then for every $\chi \in \widehat{K}$ there exists $\xi \in \widehat{G}$ such that $\xi \uparrow_{K}=\chi$.

Proof. Define $H=\left\{\chi \in \widehat{K}\right.$ : there exists $\xi \in \widehat{G}$ with $\left.\xi \uparrow_{K}=\chi\right\}$. By Theorem 6.19 the continuous characters of $G$ separate the points of $G$. Therefore $H$ separate the points of $K$. Now apply Corollary 6.6 to conclude that $H=\widehat{K}$.

Here is another corollary of Theorem 6.19:

Corollary 6.21. A $\sigma$-compact and locally compact abelian group is totally disconnected iff for every continuous character $\chi$ of $G$ the image $\chi(G)$ is a proper subgroup of $\mathbb{T}$. 
Proof. Assume that $G$ is a locally compact abelian group such that $\chi(G)$ is a proper subgroup of $\mathbb{T}$ for every continuous character $\chi$ of $G$. According to Theorem 6.19 the diagonal homomorphism $f: G \rightarrow \prod\{\chi(G): \chi \in \widehat{G}\}$ of all $\chi \in \widehat{G}$ is injective. Since the proper subgroups of $\mathbb{T}$ are totally disconnected, the whole product will be totally disconnected, so also $G$ will be totally disconnected. Now assume that $G$ is $\sigma$-compact, locally compact and totally disconnected. Consider $\chi \in \widehat{G}$ and assume for a contradiction that $\chi(G)=\mathbb{T}$. Then $\chi: G \rightarrow \mathbb{T}$ will be an open map by the open mapping theorem, so $\mathbb{T}$ will be a quotient of $G$. As total disconnectedness is inherited by quotiens of locally compact groups (see Corollary 4.20 ), we conclude that $\mathbb{T}$ must be totally disconnected, a contradiction.

Corollary 6.22. A compact abelian group is totally disconnected iff every continuous character of $G$ is torsion.

Proof. For a compact abelian group $G$ the image $\chi(G)$ under a continuous character $\chi$ of $G$ is a compact, hence closed subgroup of $\mathbb{T}$. Hence $\chi(G)$ is a proper subgroup of $\mathbb{T}$ precisely when it is finite. This means that the character $\chi$ is torsion.

Compactness plays an essential role here. We shall see examples of totally disconnected $\sigma$-compact and locally compact abelian groups $G$ such that no continuous character of $G$ is torsion (e.g., $G=\mathbb{Q}_{p}$ ).

\section{Pontryagin-van Kampen duality}

\subsection{The dual group}

In the sequel we shall write the circle additively as $(\mathbb{T},+)$ and we denote by $q_{0}: \mathbb{R} \rightarrow \mathbb{T}=\mathbb{R} / \mathbb{Z}$ the canonical projection. For every $k \in \mathbb{N}_{+}$let $\Lambda_{k}=q_{0}\left(\left(-\frac{1}{3 k}, \frac{1}{3 k}\right)\right)$. Then $\left\{\Lambda_{k}: k \in \mathbb{N}_{+}\right\}$is a base of the neighborhoods of 0 in $\mathbb{T}$, because $\left\{\left(-\frac{1}{3 k}, \frac{1}{3 k}\right): k \in \mathbb{N}_{+}\right\}$is a base of the neighborhoods of 0 in $\mathbb{R}$.

For every abelian group $G^{*}=\operatorname{Hom}(G, \mathbb{T})$. For a subset $K$ of $G$ and a subset $U$ of $\mathbb{T}$ let

$$
W_{G^{*}}(K, U)=\{\chi \in G: \chi(K) \subseteq U\}
$$

For any subgroup $H$ of $G^{*}$ we abbreviate $H \cap W(K, U)$ to $W_{H}(K, U)$. When there is no danger of confusion we shall write only $W(K, U)$ in place of $W_{G^{*}}(K, U)$. The group $G^{*}$ will be considered only with one topology, namely the induced from $\mathbb{T}^{G}$ compact topology (see Remark 4.1).

If $G$ is a topological abelian group, $\widehat{G}$ will denote the subgroup of $G^{*}$ consisting of continuous characters.

The group $\widehat{G}$ will carry the compact open topology that has as basic neighborhoods of 0 the sets $W_{\widehat{G}}(K, U)$, where $K$ is a compact subset of $G$ and $U$ is neighborhood of 0 in $\mathbb{T}$. We shall see below that when $U \subseteq \Lambda_{1}$, then $W_{\widehat{G}}(K, U)$ coincides with $W_{G^{*}}(K, U)$ in case $K$ is a neighborhood of 0 in $G$. Therefore we shall use mainly the notation $W(K, U)$ when the group $G$ is clear from the context.

Let us start with an easy example.

Example 7.1. Let $G$ be an abelian topological group.

(1) If $G$ is compact, then $\widehat{G}$ is discrete.

(2) If $G$ is discrete, then $\widehat{G}$ is compact.

Indeed, to prove (1) it is sufficient to note that $W_{\widehat{G}}\left(G, \Lambda_{1}\right)=\{0\}$ as $\Lambda_{1}$ contains no subgroup of $\mathbb{T}$ beyond 0 .

(2) Suppose that $G$ is discrete. Then $\widehat{G}=\operatorname{Hom}(G, \mathbb{T})$ is a subgroup of the compact group $\mathbb{T}^{G}$. The compactopen topology of $\widehat{G}$ coincides with the topology inherited from $\mathbb{T}^{G}$ : let $F$ be a finite subset of $G$ and $U$ an open neighborhood of 0 in $\mathbb{T}$, then

$$
\begin{aligned}
\bigcap_{x \in F} \pi_{x}^{-1}(U) \cap \operatorname{Hom}(G, \mathbb{T}) & =\left\{\chi \in \operatorname{Hom}(G, \mathbb{T}): \pi_{x} \in U \text { for every } x \in F\right\} \\
& =\{\chi \in \operatorname{Hom}(G, \mathbb{T}): \chi(x) \in U \text { for every } x \in F\}=W(F, U)
\end{aligned}
$$

Moreover $\operatorname{Hom}(G, \mathbb{T})$ is closed in the compact product $\mathbb{T}^{G}$ by Remark 4.1 and we can conclude that $\widehat{G}$ is compact.

Now we prove that the dual group is always a topological group. If the group $G$ is locally compact, then its dual is locally compact too. This is the first step of the Pontryagin-van Kampen duality theorem. 
Theorem 7.2. For an abelian topological group $G$ the following assertions hold true:

(a) if $x \in \mathbb{T}$ and $k \in \mathbb{N}_{+}$, then $x \in \Lambda_{k}$ if and only if $x, 2 x, \ldots, k x \in \Lambda_{1}$;

(b) $\chi \in \operatorname{Hom}(G, \mathbb{T})$ is continuous if and only if $\chi^{-1}\left(\Lambda_{1}\right)$ is a neighborhood of 0 in $G$;

(c) $\left\{W_{\widehat{G}}\left(K, \Lambda_{1}\right): K\right.$ compact $\left.\subseteq G\right\}$ is a base of the neighborhoods of 0 in $\widehat{G}$, in particular $\widehat{G}$ is a topological group.

(d) $W_{\widehat{G}}\left(A, \Lambda_{s}\right)+W_{\widehat{G}}\left(A, \Lambda_{s}\right) \subseteq W_{\widehat{G}}\left(A, \Lambda_{s-1}\right)$ and $W_{\widehat{G}}\left(\bar{A}, \Lambda_{s}\right)+W_{\widehat{G}}\left(\bar{A}, \Lambda_{s}\right) \subseteq W_{\widehat{G}}\left(\bar{A}, \Lambda_{s-1}\right)$ for every $A \subseteq G$ and $s>1$.

(e) if $F$ is a closed subset of $\mathbb{T}$, then for every $K \subseteq G$ the subset $W_{G^{*}}(K, F)$ of $G^{*}$ is closed (hence, compact);

(f) if $U$ is neighborhoodof 0 in $G$, then

$\left(f_{1}\right) W_{\widehat{G}}(\bar{U}, V)=W_{G^{*}}(\bar{U}, V)$ for every neighborhood of $0 V \subseteq \Lambda_{1}$ in $\mathbb{T}$;

$\left(f_{2}\right) W\left(\bar{U}, \Lambda_{4}\right)$ has compact closure;

$\left(f_{3}\right)$ if $U$ has compact closure, then $W\left(\bar{U}, \Lambda_{4}\right)$ is a neighborhood of 0 in $\widehat{G}$ with compact closure, so $\widehat{G}$ is locally compact.

Proof. (a) Note that for $s \in \mathbb{N}, s x \in \Lambda_{1}$ if and only if $x \in A_{s, t}=\Lambda_{s}+\pi_{\mathbb{T}}\left(\frac{t}{s}\right)$ for some integer $t$ with $0 \leq t \leq s$. On the other hand, $A_{s, 0}=\Lambda_{s}$ and $\Lambda_{s} \cap A_{s+1, t}$ is non-empty if and only if $t=0$. Hence, if $x \in \Lambda_{s}$ and $(s+1) x \in \Lambda_{1}$, then $x \in \Lambda_{s+1}$ and this holds in particular for $1 \leq s<k$. This proves that $s x \in \Lambda_{1}$ for $s=1, \ldots, k$ if and only if $x \in \Lambda_{k}$.

(b) Suppose that $\chi^{-1}\left(\Lambda_{1}\right)$ is a neighborhood of 0 in $G$. So there exists an open neighborhood $U$ of 0 in $G$ such that $U \subseteq \chi^{-1}\left(\Lambda_{1}\right)$. Moreover, there exists an other neighborhood $V$ of 0 in $G$ with $\underbrace{V+\cdots+V}_{k} \subseteq U$ where $k \in \mathbb{N}_{+}$. Now $s \chi(y) \in \Lambda_{1}$ for every $y \in V$ and $s=1, \ldots, k$. By item (a) $\chi(y) \in \Lambda_{k}$ and so $\chi(V) \subseteq \Lambda_{k}$.

(c) Let $k \in \mathbb{N}_{+}$and $K$ be a compact subset of $G$. Define $L=\underbrace{K+\cdots+K}_{k}$, which is a compact subset of $G$ because it is a continuous image of the compact subset $K^{k}$ of $G^{k}$. Take $\chi \in W\left(L, \Lambda_{1}\right)$. For every $x \in K$ we have $s \chi(x) \in \Lambda_{1}$ for $s=1, \ldots, k$ and so $\chi(x) \in \Lambda_{k}$ by item (a). Hence $W\left(L, \Lambda_{1}\right) \subseteq W\left(K, \Lambda_{k}\right)$.

(d) obvious.

(e) If $\pi_{x}: \mathbb{T}^{G} \rightarrow \mathbb{T}$ is the projection defined by the evaluation at $x$, for $x \in G$, then obviously

$$
W_{G^{*}}(K, F)=\bigcap_{x \in K}\left\{\chi \in G^{*}: \chi(x) \in F\right\}=\bigcap_{x \in K} \pi_{x}^{-1}(F)
$$

is cloased as each $\pi_{x}^{-1}(F)$ is closed in $G^{*}$.

$\left(\mathrm{f}_{1}\right)$ follows immediately from item $(\mathrm{c})$.

$\left(\mathrm{f}_{2}\right)$ To prove that the closure of $W_{0}=W\left(\bar{U}, \Lambda_{4}\right)$ is compact it is sufficient to note that $W_{0} \subseteq W_{1}:=W\left(\bar{U}, \overline{\Lambda_{4}}\right)$ and prove that $W_{1}$ is compact. Let $\tau_{s}$ denote the subspace topology of $W_{1}$ in $\widehat{G}$. We prove in the sequel that $\left(W_{1}, \tau_{s}\right)$ is compact.

Consider on the set $W_{1}$ also the weaker topology $\tau$ induced from $G^{*}$ and consequently from $\mathbb{T}^{G}$. By (e) $\left(W_{1}, \tau\right)$ is compact.

It remains to show that both topologies $\tau_{s}$ and $\tau$ of $W_{1}$ coincide. Since $\tau_{s}$ is finer than $\tau$, it suffices to show that if $\alpha \in W_{1}$ and $K$ is a compact subset of $G$, then $\left(\alpha+W\left(K, \Lambda_{1}\right)\right) \cap W_{1}$ is also a neighborhood of $\alpha$ in $\left(W_{1}, \tau\right)$.

Since $\bigcup\{a+U: a \in K\} \supseteq K$ and $K$ is compact, $K \subseteq F+U$, where $F$ is a finite subset of $K$. We prove now that

$$
\left(\alpha+W\left(F, \Lambda_{2}\right)\right) \cap W_{1} \subseteq\left(\alpha+W\left(K, \Lambda_{1}\right)\right) \cap W_{1} .
$$

Let $\xi \in W\left(F, \Lambda_{2}\right)$, so that $\alpha+\xi^{\prime} \in W_{1}=W\left(\bar{U}, \overline{\Lambda_{4}}\right)$. As $\alpha \in W_{1}$ as well, we deduce from items (c) and (d) that $\xi=\left(\alpha+\xi^{\prime}\right)-\alpha \in W_{1}-W_{1}$. Hence $\xi(\bar{U}) \subseteq \overline{\Lambda_{2}}$ and consequently

$$
\xi(K) \subseteq \xi(F+U) \subseteq \Lambda_{2}+\overline{\Lambda_{2}} \subseteq \Lambda_{1}
$$

This proves $\xi \in W\left(K, \Lambda_{1}\right)$ and $(*)$.

$\left(f_{3}\right)$ Follows obviously from $\left(f_{2}\right)$ and the definition of the compact open topology. 
The above proof shows another relevant fact. The neighborhood $W\left(\bar{U}, \Lambda_{4}\right)$ of 0 in the dual group $\widehat{G}$ carries the same topology in $\widehat{G}$ and $G^{*}$, nevertheless the inclusion map $j: \widehat{G} \hookrightarrow G^{*}$ need not be an embedding:

Corollary 7.3. For a locally compact abelian group $G$ the following are equivalent:

(a) the inclusion map $j: \widehat{G} \hookrightarrow G^{*}$ is an embedding;

(b) $G$ is discrete;

(c) $\widehat{G}=G^{*}$ is compact.

Proof. Since $G^{*}$ is compact, $j$ can be an embedding iff $\widehat{G}$ itself is compact. According to Example 7.1 this occurs precisely when $G$ is discrete. In that case $\widehat{G}=G^{*}$ is compact.

Actually, it can be proved, once the duality theorem is available, that $j: \widehat{G} \hookrightarrow G^{*}$ need not be even a local homeomorphism. (If $j$ is a local homeomorphism, then the topological subgroup $j(\widehat{G})$ of $G^{*}$ will be locally compact, hence closed in $G^{*}$. This would yield that $j(\widehat{G})$ is compact. On the other hand, the topology of $j(\widehat{G})$ is precisely the initial topology of all projections $p_{x}$ restricted to $\widehat{G}$. By the Pontryagin duality theorem, these projections form the group of all continuous characters of $\widehat{G}$. So this topology coincides with $\mathcal{T}_{\widehat{\widehat{G}}}$. By a general theorem of Glicksberg, a locally compact abelian groups $H$ and $\left(H, \mathcal{T}_{\widehat{H}}\right)$ have the same compact sets. In particular, compactness of $\left(H, \mathcal{T}_{\widehat{H}}\right)$ yields compactness of $H$. This proves that if $j: \widehat{G} \hookrightarrow G^{*}$ is a local homeomorphism, then $\widehat{G}$ is compact and consequently $G$ is discrete.)

\subsection{Computation of some dual groups}

In the next proprosition we show, roughly speaking, that the projective order between continuous surjective open homomorphisms with the same domain corresponds to the order by inclusion of their kernels.

Proposition 7.4. Let $G, H_{1}$ and $H_{2}$ be topological abelian groups and let $\chi_{i}: G \rightarrow H_{i}, i=1,2$, be continuous surjective open homomorphisms. Then there exists a continuous homomorphism $\iota: H_{1} \rightarrow H_{2}$ such that $\chi_{2}=$ $\iota \circ \chi_{1}$ iff $\operatorname{ker} \chi_{1} \leq \operatorname{ker} \chi_{2}$. If $\operatorname{ker} \chi_{1}=\operatorname{ker} \chi_{2}$ then $\iota$ will be a topological isomorphism.

Proof. The necessity is obvious. So assume that ker $\chi_{1} \leq \operatorname{ker} \chi_{2}$ holds. By the homomorphism theorem applied to $\chi_{i}$ there exists a topological isomorphisms $j_{i}: G / \operatorname{ker} \chi_{i} \rightarrow H_{i}$ such that $\chi_{i}=j_{i} \circ q_{i}$, where $q_{i}: G \rightarrow G / \operatorname{ker} \chi_{i}$ is the canonical homomorphism for $i=1,2$. As $\operatorname{ker} \chi_{1} \leq \operatorname{ker} \chi_{2}$ we get a continuous homomorphism $t$ that makes commutative the following diagram

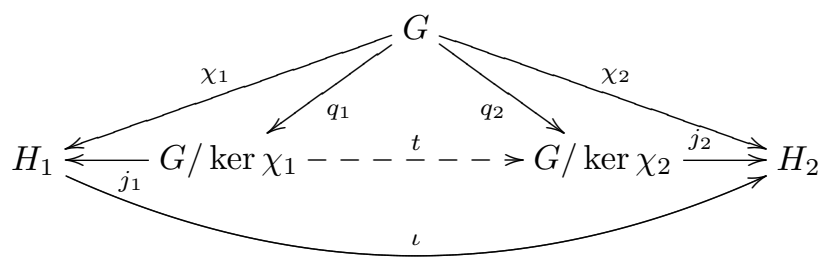

Obviously $\iota=j_{2} \circ t \circ j_{1}^{-1}$ works. If $\operatorname{ker} \chi_{1}=\operatorname{ker} \chi_{2}$, then $t$ is a topological isomorphism, hence $\iota$ will be a topological isomorphism as well.

In the sequel we denote by $k \cdot i d_{G}$ the endomorphism of an abelian group $G$ obtained by the map $x \mapsto k x$, for a fixed $k \in \mathbb{Z}$. The next lemma will be used for the computation of the dual groups in Example 7.7.

Lemma 7.5. Every continuous homomorphism $\chi: \mathbb{T} \rightarrow \mathbb{T}$ has the form $k \cdot i d_{\mathbb{T}}$, for some $k \in \mathbb{Z}$. In particular, the only topological isomorphisms $\chi: \mathbb{T} \rightarrow \mathbb{T}$ are $\pm i d_{\mathbb{T}}$.

Proof. We prove first that the only topological isomorphisms $\chi: \mathbb{T} \rightarrow \mathbb{T}$ are $\pm i d_{\mathbb{T}}$. The proof will exploit the fact that the arcs are the only connected sets of $\mathbb{T}$. Hence $\chi$ sends any $\operatorname{arc}$ of $\mathbb{T}$ to an arc, sending end points to end points. Denote by $\varphi$ the canonical homomorphism $\mathbb{R} \rightarrow \mathbb{T}$ and for $n \in \mathbb{N}$ let $c_{n}=\varphi\left(1 / 2^{n}\right)$ be the generators of the Prüfer subgroup $\mathbb{Z}\left(2^{\infty}\right)$ of $\mathbb{T}$. Then, $c_{1}$ is the only element of $\mathbb{T}$ of order 2 , hence $g\left(c_{1}\right)=c_{1}$. Therefore, the arc $A_{1}=\varphi([0,1 / 2])$ either goes onto itself, or goes onto its symmetric image $-A_{1}$. Let us consider the first case. Clearly, either $g\left(c_{2}\right)=c_{2}$ or $g\left(c_{2}\right)=-c_{2}$ as $o\left(g\left(c_{2}\right)\right)=4$ and being $\pm c_{2}$ the only elements of order 4 of $\mathbb{T}$. By our assumption $g\left(A_{1}\right)=A_{1}$ we have $g\left(c_{2}\right)=c_{2}$ since $c_{2}$ is the only element of order 4 on the arc $A_{1}$. Now the arc $A_{2}=\left[0, c_{2}\right]$ goes onto itself, hence for $c_{3}$ we must have $g\left(c_{3}\right)=c_{3}$ as the only element of order 8 on the 
$\operatorname{arc} A_{2}$, etc. We see in the same way that $g\left(c_{n}\right)=c_{n}$. Hence $g$ is identical on the whole subgroup $\mathbb{Z}\left(2^{\infty}\right)$. As this subgroup is dense in $\mathbb{T}$, we conclude that $g$ coincides with $i d_{\mathbb{T}}$. In the case $g\left(A_{1}\right)=-A_{1}$ we replace $g$ by $-g$ and the previous proof gives $-g=i d_{\mathbb{T}}$, i.e., $g=-i d_{\mathbb{T}}$.

For $k \in \mathbb{N}_{+}$let $\pi_{k}=k \cdot i d_{\mathbb{T}}$. Then $\operatorname{ker} \pi_{k}=\mathbb{Z}_{k}$ and $\pi_{k}$ is surjective. Let now $\chi: \mathbb{T} \rightarrow \mathbb{T}$ be a non-trivial continuous homomorphism. Then ker $\chi$ is a closed proper subgroup of $\mathbb{T}$, hence ker $\chi=\mathbb{Z}_{k}$ for some $k \in \mathbb{N}_{+}$. Moreover, $\chi(\mathbb{T})$ is a connected non-trivial subgroup of $\mathbb{T}$, hence $\chi(\mathbb{T})=\mathbb{T}$. By Proposition $7.4 \chi= \pm \pi_{k}$.

Obviously, $\chi= \pm \xi$ for characters $\chi, \xi: G \rightarrow \mathbb{T}$ implies $\operatorname{ker} \chi=\operatorname{ker} \xi$ and $\chi(G)=\xi(G)$. More generally, if $\chi=k \cdot \xi$ for some $k \in \mathbb{Z}$, then $\operatorname{ker} \chi \geq \operatorname{ker} \xi$ and $\chi(G) \leq \xi(G)$. Now we see that this implication can be (partially) inverted under appropriate hypotheses.

Corollary 7.6. Let $G$ be a $\sigma$-compact locally compact abelian group and let $\chi, \xi: G \rightarrow \mathbb{T}$ be continuous characters such that $\operatorname{ker} \chi \geq \operatorname{ker} \xi$ and $\chi(G) \leq \xi(G)$.

(a) If $\chi(G)=\xi(G)=\mathbb{T}$ then $\chi=k \cdot \xi$ for some $k \in \mathbb{Z}$; moreover, ker $\chi=\operatorname{ker} \xi$ iff $\chi= \pm \xi$.

(b) If $G$ is compact and $|\xi(G)|=m$ for some $m \in \mathbb{N}_{+}$, then $\chi=k \xi$ for some $k \in \mathbb{Z}$; moreover, ker $\chi=$ ker $\xi$ iff $\chi(G)=\xi(G)$, in such a case $k$ must be coprime to $m$.

(c) If $\operatorname{ker} \xi=\operatorname{ker} \xi$ is open and $H=\chi(G)=\xi(G)$, then $\chi=\iota \circ \xi$, where $\iota: H \rightarrow H$ is an arbitrary automorphism of the subgroup $H$ of $\mathbb{T}$ equipped with the discrete topology.

Proof. (a) As $\chi(G)=\xi(G)=\mathbb{T}$ and $G$ is $\sigma$-compact, we can apply Lemma 7.4 and observe that the only $\iota$ given by the lemma can be $k \cdot i d_{\mathbb{T}}$ for some $k \in \mathbb{Z}$ in view of the previous lemma. The same lemma yields $k= \pm 1$ when $\operatorname{ker} \chi=\operatorname{ker} \xi$.

(b) If $G$ is compact and $|\xi(G)|=m$ for some $m \in \mathbb{N}_{+}, \xi(G)$ is a cyclic subgroup of $\mathbb{T}$ of order $m$. Note that $\mathbb{T}$ has a unique such cyclic subgroup. By Proposition 7.4 there exists a homomorphism $\iota: \xi(G) \rightarrow \chi(G)$ such that $\chi=\iota \circ \xi$. The hypothesis $\chi(G) \leq \xi(G)$ implies that there such a $\iota$ must by the multiplication by some $k \in \mathbb{Z}$. In case $\chi(G)=\xi(G)$ this $k$ is coprime to $m$.

(c) Obvious.

Example 7.7. Let $p$ be a prime. Then $\widehat{Z\left(p^{\infty}\right)} \cong \mathbb{J}_{p}, \widehat{\mathbb{J}_{p}} \cong \mathbb{Z}\left(p^{\infty}\right), \widehat{\mathbb{T}} \cong \mathbb{Z}, \widehat{\mathbb{Z}} \cong \mathbb{T}$ and $\widehat{\mathbb{R}} \cong \mathbb{R}$.

Proof. The first isomorphism $\widehat{\mathbb{Z}\left(p^{\infty}\right)}=\mathbb{J}_{p}$ follows from our definition $\mathbb{J}_{p}=\operatorname{End}\left(\mathbb{Z}\left(p^{\infty}\right)\right)=\operatorname{Hom}\left(\mathbb{Z}\left(p^{\infty}\right), \mathbb{T}\right)=$ $\widehat{\mathbb{Z}\left(p^{\infty}\right)}$.

To verify the isomorphism $\widehat{\mathbb{J}_{p}} \cong \mathbb{Z}\left(p^{\infty}\right)$ consider first the quotient homomorphism $\eta_{n}: \mathbb{J}_{p} \rightarrow \mathbb{J}_{p} / p^{n} \mathbb{J}_{p} \cong \mathbb{Z}_{p^{n}} \leq$ $\mathbb{T}$. With this identifications we consider $\eta_{n} \in \widehat{\mathbb{J}_{p}}$. It is easy to see that under this identification $p \eta_{n}=\eta_{n-1}$. Therefore, the subgroup $H$ of $\widehat{\mathbb{J}_{p}}$ generated by the characters $\eta_{n}$ is isomorphic to $\mathbb{Z}\left(p^{\infty}\right)$. Let us see that $H=\widehat{\mathbb{J}_{p}}$. Indeed, take any non-trivial character $\chi: \mathbb{J}_{p} \rightarrow \mathbb{T}$. Then $N=\operatorname{ker} \chi$ is a closed proper subgroup of $\mathbb{J}_{p}$. Moreover, $N \neq 0$ as $\mathbb{J}_{p}$ is not isomorphic to a subgroup of $\mathbb{T}$ by Exercise 4.49 . Thus $N=p^{n} \mathbb{J}_{p}$ for some $n \in \mathbb{N}_{+}$. Since $N=\operatorname{ker} \eta_{n}$, we conclude with (b) of Corollary 7.6 that $\chi=k \eta_{n}$ for some $k \in \mathbb{Z}$. This proves that $\chi=H$ and consequently $\widehat{\mathbb{J}_{p}} \cong \mathbb{Z}\left(p^{\infty}\right)$.

The isomorphism $g: \widehat{\mathbb{Z}} \rightarrow \mathbb{T}$ is obtained by setting $g(\chi):=\chi(1)$ for every $\chi: \mathbb{Z} \rightarrow \mathbb{T}$. It is easy to check that this isomorphism is topological.

According to 7.5 every $\chi \in \widehat{\mathbb{T}}$ has the form $\chi=k \cdot i d_{\mathbb{T}}$ for some $k \in \mathbb{Z}$. This gives a homomorphism $\widehat{\mathbb{T}} \rightarrow \mathbb{Z}$ assigning $\chi \mapsto k$. It is obviously injective and surjective. This proves $\widehat{\mathbb{T}} \cong \mathbb{Z}$ since both groups are discrete.

To prove $\widehat{\mathbb{R}} \cong \mathbb{R}$ consider the character $\chi_{1}: \mathbb{R} \rightarrow \mathbb{T}$ obtained simply by the canonical map $\mathbb{R} \rightarrow \mathbb{R} / \mathbb{Z}$. For every non-zero $r \in \mathbb{R}$ consider the map $\rho_{r}: \mathbb{R} \rightarrow \mathbb{R}$ defined by $\rho_{r}(x)=r x$. Then its composition $\chi_{r}=\chi_{1} \circ \rho_{r}$ with $\chi_{1}$ gives a continuous character of $\mathbb{R}$ that is surjective and ker $\chi_{r}=\langle 1 / r\rangle$. Now consider any continuous non-trivial character $\chi \in \widehat{\mathbb{R}}$. Then $\chi$ is surjective and $N=\operatorname{ker} \chi$ is a proper closed subgroup of $\mathbb{R}$. Hence $N$ is cyclic by Exercise 3.20. Let $N=\langle 1 / r\rangle$. Then ker $\chi=\operatorname{ker} \chi_{r}$, so that Corollary 7.6 yields $\chi= \pm \chi_{r}$. The assignment $\chi \mapsto \pm r$ defines a homomorphism $\widehat{\mathbb{R}} \rightarrow \mathbb{R}$ that is obviously injective and surjective. Its continuity immediately follows from the definition of the compact-open topology of $\widehat{\mathbb{R}}$. As $\mathbb{R}$ is $\sigma$-compact, this isomorphism is also open by the open mapping theorem.

Exercise 7.8. Let $G$ be an abelian group and $p$ be a prime. Prove that

(a) $\chi \in p \widehat{G}$ iff $\chi(G[p])=0$.

(b) $p \chi=0$ in $\widehat{G}$ iff $\chi(p G)=0$. 
Conclude that

(i) a discrete abelian group $G$ is divisible (resp., torsion-free) iff $\widehat{G}$ is torsion-free (resp., divisible).

(ii) the groups $\widehat{\mathbb{Q}}$ and $\widehat{\mathbb{Q}_{p}}$ are torsion-free and divisible.

Exercise 7.9. Let $G$ be a totally disconnected locally compact abelian group. Prove that ker $\chi$ is an open subgroup of $G$ for every $\chi \in \widehat{G}$.

(Hint. Use the fact that by the continuity of $\chi$ and the total disconnectedness of $G$ there exists an open subgroup $O$ of $G$ such that $\chi(O) \subseteq \Lambda_{1}$.)

Exercise 7.10. Let $p$ be a prime. Prove that $\widehat{\mathbb{Q}_{p}} \cong \mathbb{Q}_{p}$, where $\mathbb{Q}_{p}$ denotes the field of all $p$-adic numbers.

(Hint. Fix $N=\left\{\chi \in \widehat{\mathbb{Q}}_{p}: \operatorname{ker} \chi \geq \mathbb{J}_{p}\right\}$. By the compactness of $\mathbb{J}_{p}$, conclude that $N$ is an open subgroup of $\widehat{\mathbb{Q}}_{p}$ topologically isomorphic to $\mathbb{J}_{p}$ using Exercise 7.9 and Corollary 7.6 (c). For every $n \in \mathbb{N}_{+}$let $\xi_{n}: \mathbb{Q}_{p} \rightarrow \mathbb{Q}_{p} / p^{n} \mathbb{J}_{p}$ be the canonical homomorphism. As $\mathbb{Q}_{p} / p^{-n} \mathbb{J}_{p} \cong \mathbb{Z}\left(p^{\infty}\right) \leq \mathbb{T}$, we can consider $\xi_{n} \in \widehat{\mathbb{Q}}_{p}$. Show that $p \xi_{n+1}=\xi_{n}$ for $n \in \mathbb{N}_{+}$and $p \xi_{1} \in N$. The subgroup of $\widehat{\mathbb{Q}}_{p}$ generated by $N$ and $\left(\xi_{n}\right)$ is isomorphic to $\mathbb{Q}_{p}$. Using Corollary 7.6 (c) and Exercise 7.9 deduce that it coincides with the whole group $\widehat{\mathbb{Q}}_{p}$.)

Exercise 7.11. Let $H$ be a subgroup of $\mathbb{R}^{n}$. Prove that every $\chi \in \widehat{H}$ extends to a continuous character of $\mathbb{R}^{n}$.

\subsection{Some general properties of the dual}

We prove next that the dual group of a finite product of abelian topological groups is the product of the dual groups of each group.

Lemma 7.12. If $G$ and $H$ are topological abelian groups, then $\widehat{G \times H}$ is isomorphic to $\widehat{G} \times \widehat{H}$.

Proof. Define $\Phi: \widehat{G} \times \widehat{H} \rightarrow \widehat{G \times H}$ by $\Phi\left(\chi_{1}, \chi_{2}\right)\left(x_{1}, x_{2}\right)=\chi_{1}\left(x_{1}\right)+\chi_{2}\left(x_{2}\right)$ for every $\left(\chi_{1}, \chi_{2}\right) \in \widehat{G} \times \widehat{H}$ and $\left(x_{1}, x_{2}\right) \in G \times H$. Then $\Phi$ is a homomorphism, in fact $\Phi\left(\chi_{1}+\psi_{1}, \chi_{2}+\psi_{2}\right)\left(x_{1}, x_{2}\right)=\left(\chi_{1}+\psi_{1}\right)\left(x_{1}\right)+\left(\chi_{2}+\psi_{2}\right)\left(x_{2}\right)=$ $\chi_{1}\left(x_{1}\right)+\psi_{1}\left(x_{1}\right)+\chi_{2}\left(x_{2}\right)+\psi_{2}\left(x_{2}\right)=\Phi\left(\chi_{1}, \chi_{2}\right)\left(x_{1}, x_{2}\right)+\Phi\left(\psi_{1}, \psi_{2}\right)\left(x_{1}, x_{2}\right)$.

Moreover $\Phi$ is injective, because

$$
\begin{aligned}
\operatorname{ker} \Phi & =\{(\chi, \psi) \in \widehat{G} \times \widehat{H}: \Phi(\chi, \psi)=0\} \\
& =\{(\chi, \psi) \in \widehat{G} \times \widehat{H}: \Phi(\chi, \psi)(x, y)=0 \text { for every }(x, y) \in G \times H\} \\
& =\{(\chi, \psi) \in \widehat{G} \times \widehat{H}: \chi(x)+\psi(y)=0 \text { for every }(x, y) \in G \times H\} \\
& =\{(\chi, \psi) \in \widehat{G} \times \widehat{H}: \chi(x)=0 \text { and } \psi(x)=0 \text { for every }(x, y) \in G \times H\} \\
& =\{(0,0)\} .
\end{aligned}
$$

To prove that $\Phi$ is surjective, take $\psi \in \widehat{G \times H}$ and note that $\psi\left(x_{1}, x_{2}\right)=\psi\left(x_{1}, 0\right)+\psi\left(0, x_{2}\right)$. Now define $\psi_{1}\left(x_{1}\right)=\psi\left(x_{1}, 0\right)$ for every $x_{1} \in G$ and $\psi_{2}\left(x_{2}\right)=\psi\left(0, x_{2}\right)$ for every $x_{2} \in H$. Hence $\psi_{1} \in \widehat{G}, \psi_{2} \in \widehat{H}$ and $\psi=\Phi\left(\psi_{1}, \psi_{2}\right)$.

Now we show that $\Phi$ is continuous. Let $W(K, U)$ be an open neighborhood of 0 in $\widehat{G \times H}(K$ is a compact subset of $G \times H$ and $U$ is an open neighborhood of 0 in $\mathbb{T}$ ). Since the projections $\pi_{G}$ and $\pi_{H}$ of $G \times H$ onto $G$ and $H$ are continuous, $K_{G}=\pi_{G}(K)$ and $K_{H}=\pi_{H}(K)$ are compact in $G$ and in $H$ respectively. Taking an open symmetric neighborhood $V$ of 0 in $\mathbb{T}$, it follows $\Phi\left(W\left(K_{G}, V\right) \times W\left(K_{H}, V\right)\right) \subseteq W(K, U)$.

It remains to prove that $\Phi$ is open. Consider two open neighborhoods $W\left(K_{G}, U_{G}\right)$ of 0 in $\widehat{G}$ and $W\left(K_{H}, U_{H}\right)$ of 0 in $\widehat{H}$, where $K_{G} \subseteq G$ and $K_{H} \subseteq H$ are compact and $U_{G}, U_{H}$ are open neighborhoods of 0 in $\mathbb{T}$. Then $K=\left(K_{G} \cup\{0\}\right) \times\left(K_{H} \cup\{0\}\right)$ is a compact subset of $G \times H$ and $U=U_{G} \cap U_{H}$ is an open neighborhood of 0 in $\mathbb{T}$. Thus $W(K, U) \subseteq \Phi\left(W\left(K_{G}, U_{G}\right) \times W\left(K_{H}, U_{H}\right)\right)$, because if $\chi \in W(K, U)$ then $\chi=\Phi\left(\chi_{1}, \chi_{2}\right)$, where $\chi_{1}\left(x_{1}\right)=\chi\left(x_{1}, 0\right) \in U \subseteq U_{G}$ for every $x_{1} \in G$ and $\chi_{2}\left(x_{2}\right)=\chi\left(0, x_{2}\right) \in U \subseteq U_{H}$ for every $x_{2} \in H$.

It follows from Proposition 7.7 that the groups $\mathbb{T}, \mathbb{Z}, \mathbb{Z}\left(p^{\infty}\right), \mathbb{J}_{p}$ e $\mathbb{R}$ satisfy $\widehat{\widehat{G}} \cong G$, namely the Pontryaginvan Kampen duality theorem. Using the next theorem this propertiy extends to all finite direct products of these groups.

Call a topological abelian group $G$ autodual, if $G$ satisfies $\widehat{G} \cong G$. We have seen already that $\mathbb{R}$ and $\mathbb{Q}_{p}$ are autodual. By Lemma 7.12 finite direct products of autodual groups are autodual. Now using this observation and Lemma 7.12 we provide a large supply of groups for which the Pontryagin-van Kampen duality holds true. 
Proposition 7.13. Let $P_{1}, P_{2}$ and $P_{3}$ be finite sets of primes, $m, n, k, k_{p} \in \mathbb{N}\left(p \in P_{3}\right)$ and $n_{p}, m_{p} \in \mathbb{N}_{+}$ $\left(p \in P_{1} \cup P_{2}\right)$. Then every group of the form

$$
G=\mathbb{T}^{n} \times \mathbb{Z}^{m} \times \mathbb{R}^{k} \times F \times \prod_{p \in P_{1}} \mathbb{Z}\left(p^{\infty}\right)^{n_{p}} \times \prod_{p \in P_{2}} \mathbb{J}_{p}^{m_{p}} \times \prod_{j \in P_{3}} \mathbb{Q}_{p}^{k_{p}},
$$

where $F$ is a finite abelian group, satisfies $\widehat{\widehat{G}} \cong G$.

Moreover, such a group is autodual iff $n=m, P_{1}=P_{2}$ and $n_{p}=m_{p}$ for all $p \in P_{1}=P_{2}$. In particular, $\widehat{\widehat{G}} \cong G$ holds true for all elementary locally compact abelian groups.

Proof. Let us start by proving $\widehat{F}=F^{*} \cong F$. Recall that $F$ has the form $F \cong \mathbb{Z}_{n_{1}} \times \ldots \times \mathbb{Z}_{n_{m}}$. So applying Theorem 7.14 we are left with the proof of the isomorphism $\mathbb{Z}_{n}^{*} \cong \mathbb{Z}_{n}$ for every $n \in \mathbb{N}_{+}$. The elements $x$ of $\mathbb{T}$ satisfying $n x=0$ are precisely those of the unique cyclic subgroup of order $n$ of $\mathbb{T}$, we shall denote that subgroup by $\mathbb{Z}_{n}$. Therefore, the group $\operatorname{Hom}\left(\mathbb{Z}_{n}, \mathbb{Z}_{n}\right)$ of all homomorphisms $\mathbb{Z}_{n} \rightarrow \mathbb{Z}_{n}$ is isomorphic to $\mathbb{Z}_{n}$.

It follows easily from Lemma 7.12 that if $\widehat{G}_{i} \cong G_{i}$ (resp., $\widehat{G}_{i} \cong G_{i}$ ) for a finite family $\left\{G_{i}\right\}_{i=1}^{n}$ of topological abelian groups, then also $G=\prod_{i=1}^{n} G_{i}$ satisfies $\widehat{\widehat{G}} \cong G$ (resp., $\widehat{G} \cong G$ ). Therefore, it suffices to verify that the groups $\mathbb{T}, \mathbb{Z}, \mathbb{Z}\left(p^{\infty}\right)$, and $\mathbb{J}_{p}$ e satisfy $\widehat{\widehat{G}} \cong G$, while $\widehat{\mathbb{R}} \cong \mathbb{R}, \widehat{\mathbb{Q}_{p}} \cong \mathbb{Q}_{p}$ were already checked.

It follows from Proposition 7.7 that $\widehat{\mathbb{Z}} \cong \mathbb{T}$ and $\widehat{\mathbb{T}} \cong \mathbb{Z}$, hence $\mathbb{Z} \cong \widehat{\mathbb{Z}}$ and $\mathbb{T} \cong \widehat{\widehat{\mathbb{T}}}$. Analogously, $\widehat{\mathbb{Z}\left(p^{\infty}\right)} \cong \mathbb{J}_{p}$ and $\widehat{\mathbb{J}_{p}} \cong \mathbb{Z}\left(p^{\infty}\right)$ yield $\mathbb{Z}\left(p^{\infty}\right) \cong \widehat{\widehat{\mathbb{Z}\left(p^{\infty}\right)}}$ and $\mathbb{J}_{p} \cong \widehat{\widehat{\mathbb{J}}_{p}}$.

The problem of characterizing all autodual locally compact abelian groups is still open [47, 48].

Theorem 7.14. Let $\left\{D_{i}\right\}_{i \in I}$ be a family of discrete abelian groups and let $\left\{G_{i}\right\}_{i \in I}$ be a family of compact abelian groups. Then

$$
\widehat{\bigoplus_{i \in I} D_{i}} \cong \prod_{i \in I} \widehat{D_{i}} \quad \text { and } \quad \widehat{\prod_{i \in I} G_{i}} \cong \bigoplus_{i \in I} \widehat{G_{i}}
$$

Proof. Let $\chi: \bigoplus_{i \in I} D_{i} \rightarrow \mathbb{T}$ be a character and let $\chi_{i}: D_{i} \rightarrow \mathbb{T}$ be its restriction to $D_{i}$. Then $\chi \mapsto\left(\chi_{i}\right) \in$ $\prod_{i \in I} \widehat{D_{i}}$ is the first isomorphism in $(5)$.

Let $\chi: \prod_{i \in I} G_{i} \rightarrow \mathbb{T}$ be a continuous character. Pick a neighborhood $U$ of 0 containing no non-trivial subgroups of $\mathbb{T}$. Then there exists a neighborhood $V$ of 0 in $G=\prod_{i \in I} G_{i}$ with $\chi(V) \subseteq U$. By the definition of the Tychonov topology there exists a finite subset $F \subseteq I$ such that $V$ contains the subproduct $B=\prod_{i \in I \backslash F} G_{i}$. Being $\chi(B)$ a subgroup of $\mathbb{T}$, we conclude that $\chi(B)=0$ by the choice of $U$. Hence $\chi$ factorizes through the projection $p: G \rightarrow \prod_{i \in F} G_{i}=G / B$; so there exists a character $\chi^{\prime}: \prod_{i \in F} G_{i} \rightarrow \mathbb{T}$ such that $\chi=\chi^{\prime} \circ p$. Obviously, $\chi^{\prime} \in \oplus_{i \in I} \widehat{G_{i}}$. Then $\chi \mapsto \chi^{\prime}$ is the second isomorphism in (5).

In order to extend the isomorphism (5) to the general case of locally compact abelian groups one has to consider a specific topology on the direct sum.

Algebraic properties of the dual group $\widehat{G}$ of a compact abelian group $G$ can be described in terms of topological properties of the group $G$. We saw in Corollary 6.22 that $\widehat{G}$ is torsion precisely when $G$ is totally disconnected. Here is the counterpart of this property in the connected case:

Proposition 7.15. Let $G$ be a topological abelian group.

(a) If $G$ is connected, then the dual group $\widehat{G}$ is torsion-free.

(b) If $G$ is compact, then the dual group $\widehat{G}$ is torsion-free iff $G$ is connected.

Proof. (a) Since for every non-zero continuous character $\chi: G \rightarrow \mathbb{T}$ the image $\chi(G)$ is a non-trivial connected subgroup of $\mathbb{T}$, we deduce that $\chi(G)=\mathbb{T}$ for every non-zero $\chi \in \widehat{G}$. Hence $\widehat{G}$ is torsion-free.

(b) If the group $G$ is compact and disconnected, then by Theorem 4.19 there exists a proper open subgroup $N$ of $G$. Take any non-zero character $\xi$ of the finite group $G / N$. Then $m \xi=0$ for some positive integer $m$. Now the composition $\chi$ of $\xi$ and the canonical homomorphism $G \rightarrow G / N$ satisfies $m \chi=0$ as well. So $\widehat{G}$ haas a non-zero torsion character. This proves the implication left open by item (a).

Let $G$ and $H$ be abelian topological groups. If $f: G \rightarrow H$ is a continuous homomorphism, define $\widehat{f}: \widehat{H} \rightarrow \widehat{G}$ putting $\widehat{f}(\chi)=\chi \circ f$ for every $\chi \in \widehat{H}$. 
Lemma 7.16. If $f: G \rightarrow H$ is a continuous homomorphism of topological abelian group, then $\widehat{f}(\chi)=\chi \circ f$ is a continuous homomorphism as well.

(a) If $f(G)$ is dense in $H$, then $\widehat{f}$ is injective.

(b) If $f$ is injective and $f(G)$ is either open or dense in $H$, then $\widehat{f}$ is surjective.

(c) if $f$ is a surjective homomorphism, such that every compact subset of $H$ is covered by some compact subset of $G$, then $\widehat{f}$ is an embedding.

(d) if $f$ is a quotient homomorphism and $G$ is locally compact, then $\widehat{f}$ is an embedding.

(e) If $f$ is a topological isomorphism, then $\widehat{f}$ is a topological isomorphism too.

Proof. Assume $K$ is a compact subset of $G$ and $U$ a neighborhood of 0 in $\mathbb{T}$. Then $f(K)$ is a compact set in $H$, so $W=W_{\widehat{G}}(f(K), U)$ is a neighborhood of 0 in $\widehat{H}$ and $\widehat{f}(W) \subseteq W(K, U)$. This proves the continuity of $\widehat{f}$.

(a) If $\widehat{f}(\chi)=0$, then $\chi \circ f=0$. By the density of $f(G)$ in $H$ this yields $\chi=0$.

(b) Let $\chi \in \widehat{G}$. If $f(G)$ is open in $H$, then any extension $\xi: H \rightarrow \mathbb{T}$ of $\chi$ will be continuous on $f(G)$. There exists at least one such extension $\xi$ by Corollary 2.6. Hence $\xi \in \widehat{H}$ and $\chi=\widehat{f}(\xi)$. Now consider the case when $f(G)$ is dense in $H$. Then $\widetilde{H}=\widetilde{G}$ and the characters of $H$ can be extended to characters of $G$ (see Theorem 3.79).

(c) Assume $L$ is a compact subset of $G / H$ and $U$ a neighborhood of 0 in $\mathbb{T}$. Let $K$ be a compact set in $G$ such that $f(K)=L$. Then $\widehat{f}\left(W_{\widehat{H}}(L, U)\right)=\operatorname{Im} \widehat{f} \cap W_{\widehat{G}}(K, U)$, so $\widehat{f}$ is an embedding.

(d) Follows from (c) and Lemma 4.6.

(e) Obvious.

Exercise 7.17. Prove that $\widehat{\mathbb{Q} / \mathbb{Z}} \cong \prod_{p} \mathbb{J}_{p}$.

(Hint. Use the isomorphism $\mathbb{Q} / \mathbb{Z} \cong \bigoplus_{p} \mathbb{Z}\left(p^{\infty}\right)$, Example 7.7 and Theorem 7.14.)

Now we shall see that the group $\mathbb{Q}$ satisfies the duality theorem (see item (b) below).

Example 7.18. Let $K$ denote the compact group $\widehat{\mathbb{Q}}$. Then:

(a) $K$ contains a closed subgroup $H$ isomorphic to $\widehat{\mathbb{Q} / \mathbb{Z}}$ such that $K / H \cong \mathbb{T}$;

(ii) $\widehat{K} \cong \mathbb{Q}$.

(a) Denote by $H$ the subgroup of all $\chi \in K$ such that $\chi(\mathbb{Z})=0$. To prove that $H$ is a closed subgroup of $K$ such that $K / H$ is isomorphic to $\mathbb{T}$. To this end consider the continuous map $\rho: K \rightarrow \widehat{\mathbb{Z}}$ obtained by the restriction

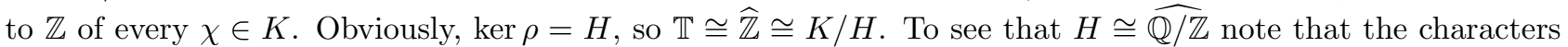
of $\mathbb{Q} / \mathbb{Z}$ correspond precisely to those characters of $\mathbb{Q}$ that vanish on $\mathbb{Z}$, i.e., precisely $H$.

(b) By Exercise $7.8 K$ is a divisible torsion-free group, every non-zero $r \in \mathbb{Q}$ defines a continuous automorphism $\lambda_{r}$ of $K$ by setting $\lambda_{r}(x)=r x$ for every $x \in K$. Then the composition $\rho \circ \lambda_{r}: K \rightarrow \mathbb{T}$ defines a character

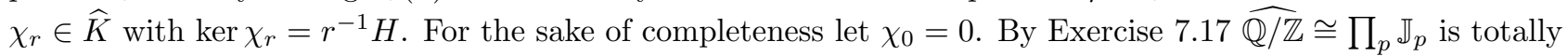
disconnected, so by Corollary $6.21 H$ has no surjective characters $\chi: H \rightarrow \mathbb{T}$. Now let $\chi \in \widehat{K}$ be non-zero. Then $\chi(K)$ will be a non-zero closed divisible subgroup of $\mathbb{T}$, hence $\chi(K)=\mathbb{T}$. On the other hand, $N=\operatorname{ker} \chi$ is a proper closed subgroup of $K$ such that $N+H \neq \mathbb{T}$, as $\chi(H)$ is a proper closed subgroup of $\mathbb{T}$ by the previous argument. Hence, $\chi(H)$ is finite, say of order $m$. Then $N+H$ contains $N$ is a finite-index subgroup, more precisely $[H:(N \cap H)]=[(N+H): N]=m$. Then $m H \leq N$. Consider the character $\chi_{m^{-1}}$ of $K$ having ker $\chi_{m^{-1}}=m H \leq N$. By Corollary there exists $k \in \mathbb{Z}$ such that $\chi=k \chi_{m^{-1}}=\chi_{r}$, where $r=k m^{-1} \in \mathbb{Q}$. This shows that $\widehat{K}=\left\{\chi_{r}: r \in \mathbb{Q}\right\} \cong \mathbb{Q}$.)

The compact group $\widehat{\mathbb{Q}}$ is closely related to the adele rings of the field $\mathbb{Q}$, more detail can be found in $[34,38,75,97]$.

Exercise 7.19. Prove that a discrete abelian group $G$ satisfies $\widehat{\widehat{G}} \cong G$ whenever 
(a) $G$ is divisible;

(b) $G$ is free;

(c) $G$ is of finite exponent;

(d) $G$ is torsion and every primary component of $G$ is of finite exponent.

(Hint. (a) Use Examples 7.7 and 7.18 (b) and the fact that every divisible group is a direct sum of copies of $\mathbb{Q}$ and the groups $\mathbb{Z}\left(p^{\infty}\right)$.

(c) and (d) Use that fact that every abelian group of finite exponent is a direct sum of cyclic subgroups (i.e., Prüfer's theorem, see (d) of Example 2.3).

Exercise 7.20. Prove that every torsion compact abelian group $G$ is bounded. More precisely, there exists natural numbers $m_{1}, \ldots, m_{n}$ and cardinals $\alpha_{1}, \ldots, \alpha_{n}$ such that $G \cong \prod_{i=1}^{n} \mathbb{Z}\left(m_{i}\right)^{\alpha_{i}}$.

(Hint. Use the Baire category theorem for the union $G=\bigcup_{n=1}^{\infty} G[n !]$ of closed subgroups. Conclude that $G[n !]$ is open for some $n$, so must have finite index by the compactness of $G$. This yields $m G=0$ for some $m$. Show that this yields also $m \widehat{G}=0$. Now apply Prüfer's theorem to $\widehat{G}$ and the fact that $G \cong \widehat{\widehat{G}}$.)

\subsection{The natural transformation $\omega$}

Let $G$ be a topological abelian group. Define $\omega_{G}: G \rightarrow \widehat{\widehat{G}}$ such that $\omega_{G}(x)(\chi)=\chi(x)$, for every $x \in G$ and for every $\chi \in \widehat{G}$. We show now that $\omega_{G}(x) \in \widehat{\widehat{G}}$.

Proposition 7.21. If $G$ is a topological abelian group. Then $\omega_{G}(x) \in \widehat{\widehat{G}}$ and $\omega_{G}: G \rightarrow \widehat{\widehat{G}}$ is a homomorphism. If $G$ is locally compact, then the homomorphism $\omega_{G}$ is a continuous.

Proof. In fact,

$$
\omega_{G}(x)(\chi+\psi)=(\chi+\psi)(x)=\chi(x)+\psi(x)=\omega_{G}(x)(\chi)+\omega_{G}(x)(\psi),
$$

for every $\chi, \psi \in \widehat{G}$. Moreover, if $U$ is an open neighborhood of 0 in $\mathbb{T}$, then $\omega_{G}(x)(W(\{x\}, U)) \subseteq U$. This proves that $\omega_{G}(x)$ is a character of $\widehat{G}$, i.e., $\omega_{G}(x) \in \widehat{\widehat{G}}$. For every $x, y \in G$ and for every $\chi \in \widehat{G}$ we have $\omega_{G}(x+y)(\chi)=(\chi)(x+y)=\chi(x)+\chi(y)=\omega_{G}(\chi)(x)+\omega_{G}(\chi)(y)$ and so $\omega_{G}$ is a homomorphism.

Now assume $G$ is locally compact. To prove that $\omega_{G}$ is continuous, pick an open neighborhood $A$ of 0 in $\mathbb{T}$ and a compact subset $K$ of $\widehat{G}$. Then $W(K, A)$ is an open neighborhood of 0 in $\widehat{\widehat{G}}$. Let $U$ be an open neighborhood of 0 in $G$ with compact closure. Take an open symmetric neighborhood $B$ of 0 in $\mathbb{T}$ with $B+B \subseteq A$. Thus $W(\bar{U}, B)$ is an open neighborhood of 0 in $\widehat{G}$. Since $K$ is compact, there exist finitely many characters $\chi_{1}, \ldots, \chi_{m}$ of $G$ such that $K \subseteq\left(\chi_{1}+W(\bar{U}, B)\right) \cup \cdots \cup\left(\chi_{m}+W(\bar{U}, B)\right)$. For every $i=1, \ldots, m$ there is an open neighborhood $V_{i}$ of 0 in $G$ such that $\chi_{i}\left(V_{i}\right) \subseteq B$. Define $V=U \cap V_{1} \cap \cdots \cap V_{m} \subseteq U$ and note that $\chi_{i}(V) \subseteq B$ for every $i=1, \ldots, m$. Thus $\omega_{G}(V) \subseteq W(K, A)$. Indeed, if $x \in V$ and $\chi \in K$, then $\chi_{i}(x) \in B$ for every $i=1, \ldots, m$ and there exists $i_{0} \in\{1, \ldots, m\}$ such that $\chi \in \chi_{i_{0}}+W(\bar{U}, B)$; so $\chi(x)=\chi_{i_{0}}(x)+\psi(x)$ with $\psi \in W(\bar{U}, B)$ and then $\omega_{G}(x)(\chi)=\chi(x) \in B+B \subseteq A$.

In this chapter we shall have a precise approach, by saying that a group $G$ satisfies the Pontryagin-van Kampen duality theorem when $\omega_{G}$ is a topological isomorphism.

Lemma 7.22. If the topological abelian groups $G_{i}$ satisfy Pontryagin-van Kampen duality theorem for $i=$ $1,2, \ldots, n$, then also $G=\prod_{i=1}^{n} G_{i}$ satisfies Pontryagin-van Kampen duality theorem.

Proof. Apply Lemma 7.12 twice to obtain an isomorphism $j: \prod_{i=1}^{n} \widehat{\widehat{G}}_{i} \rightarrow \widehat{\widehat{G}}$. It remains to verify that the product $\pi: G \rightarrow \prod_{i=1}^{n} \widehat{\widehat{G}}_{i}$ of the isomorphisms $\omega_{G_{i}}: G_{i} \rightarrow \prod_{i=1}^{n} \widehat{\widehat{G}}_{i}$ given by our hypothesis composed with the isomorphism $j$ gives precisely $\omega_{G}$.

Consider two categories $\mathcal{A}$ and $\mathcal{B}$. A covariant [contravariant] functor $F: \mathcal{A} \rightarrow \mathcal{B}$ assigns to each object $A \in \mathcal{A}$ an object $F A \in \mathcal{B}$ and to each arrow $f: A \rightarrow A^{\prime}$ in $\mathcal{A}$ an arrow $F f: F A \rightarrow F A^{\prime}\left[F f: F A^{\prime} \rightarrow F A\right]$ such that $F i d_{A}=i d_{F A}$ and $F(g \circ f)=F g \circ F f[F(g \circ f)=F f \circ F g]$ for every arrow $f: A \rightarrow A^{\prime}$ and $g: A^{\prime} \rightarrow A^{\prime \prime}$ in $\mathcal{A}$. 
Let $F, F^{\prime}: \mathcal{A} \rightarrow \mathcal{B}$ be covariant functors. A natural transformation $\gamma$ from $F$ to $F^{\prime}$ assigns to each $A \in \mathcal{A}$ an arrow $\gamma_{A}: F A \rightarrow F^{\prime} A$ such that for every arrow $f: A \rightarrow A^{\prime}$ in $\mathcal{A}$ the following diagram is commutative

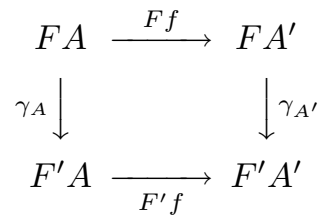

A natural equivalence is a natural transformation $\gamma$ such that each $\gamma_{A}$ is an isomorphism.

If $\mathcal{H}$ denote the category of all Hausdorff abelian topological groups, the Pontryagin-van Kampen duality functor, defined by

$$
G \mapsto \widehat{G} \text { and } f \mapsto \widehat{f}
$$

for objects $G$ and morphisms $f$ of $\mathcal{H}$, is a contravariant functor $\widehat{\uparrow}: \mathcal{H} \rightarrow \mathcal{H}$. Let $\mathcal{L}$ be the full subcategory of $\mathcal{H}$

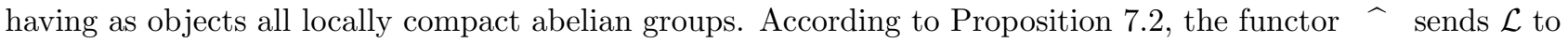
itself, i.e., defines a functor $\widehat{\imath}: \mathcal{L} \rightarrow \mathcal{L}$. The Pontryagin-van Kampen duality theorem states that $\omega$ is a natural equivalence from $i d_{\mathcal{L}}$ to $\widehat{\widehat{\wedge}}: \mathcal{L} \rightarrow \mathcal{L}$. We start by proving that $\omega$ is a natural transformation.

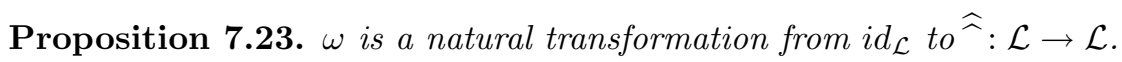

Proof. By Proposition $7.21 \omega_{G}$ is continuous for every $G \in \mathcal{L}$. Moreover for every continuous homomorphism $f: G \rightarrow H$ the following diagram commutes:

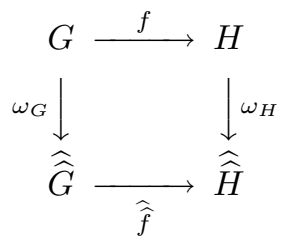

In fact, if $x \in G$ and $\xi \in \widehat{H}$, then $\omega_{H}(f(x))(\xi)=\xi(f(x))$. On the other hand,

$$
\left(\widehat{\hat{f}}\left(\omega_{G}(x)\right)\right)(\xi)=\left(\omega_{G}(x) \circ \widehat{f}\right)(\xi)=\omega_{G}(x)(\widehat{f}(\xi))=\omega_{G}(x)(\xi \circ f)=\xi(f(x)) .
$$

Hence $\omega_{H}(f(x))=\widehat{\widehat{f}}\left(\omega_{G}(x)\right)$ for every $x \in G$.

Remark 7.24. Note that $\omega_{G}$ is a monomorphism if and only if $\widehat{G}$ separates the points of $G$. Moreover, $\omega_{G}(G)$ is a subgroup of $\widehat{\widehat{G}}$ that separates the points of $\widehat{G}$.

Now we can prove the Pontryagin-van Kampen duality theorem in the case when $G$ is either compact or discrete.

Theorem 7.25. If the abelian topological group $G$ is either compact or discrete, then $\omega_{G}$ is a topological isomorphism.

Proof. If $G$ is discrete, then $\widehat{G}$ separates the points of $G$ by Corollary 2.7 and if $G$ is compact, then $\widehat{G}$ separates the points of $G$ by the Peter-Weyl Theorem 6.4. Therefore $\omega_{G}$ is injective by Remark 7.24. If $G$ is discrete, then $\widehat{G}$ is compact and $\omega_{G}(G)=\widehat{\widehat{G}}$ by Corollary 6.6. Since $\widehat{\widehat{G}}$ is discrete, $\omega_{G}$ is a topological isomorphism.

Let now $G$ be compact. Then $\omega_{G}$ is open thanks to Theorem 4.9. Suppose that $\omega_{G}(G)$ is a proper subgroup of $\widehat{\widehat{G}}$. By the compactness of $G, \widehat{\widehat{G}}$ is compact, hence closed in $\widehat{\widehat{G}}$. By the Peter-Weyl Theorem 6.4 applied to $\widehat{\widehat{G}} / \omega_{G}(G)$, there exists $\xi \in \widehat{\widehat{G}} \backslash\{0\}$ such that $\xi\left(\omega_{G}(G)\right)=\{0\}$. Since $\widehat{G}$ is discrete, $\omega_{\widehat{G}}$ is a topological isomorphism and so there exists $\chi \in \widehat{G}$ such that $\omega_{\widehat{G}}(\chi)=\xi$. Thus for every $x \in G$ we have $0=\xi\left(\omega_{G}(x)\right)=$ $\omega_{\widehat{G}}(\chi)\left(\omega_{G}(x)\right)=\omega_{G}(x)(\chi)=\chi(x)$. It follows that $\chi \equiv 0$ and so that also $\xi \equiv 0$, a contradiction.

Our next step is to prove the Pontryagin-van Kampen duality theorem when $G$ is elementary locally compact abelian:

Theorem 7.26. If $G$ is an elementary locally compact abelian group, then $\omega_{G}$ is a topological isomorphism of G onto $\widehat{\widehat{G}}$. 
Proof. According to Lemma 7.22 and Theorem 7.25 it suffices to prove that $\omega_{\mathbb{R}}$ is a topologically isomorphism. Of course, by the fact that $\widehat{\mathbb{R}}$ is topologically isomorphic to $\mathbb{R}$, one concludes immediately that also $\mathbb{R}$ and $\widehat{\mathbb{R}}$ are topologically isomorphism. A more careful analysis of the dual $\widehat{\mathbb{R}}$ shows the crucial role of the $(\mathbb{Z}$-)bilnear map $\lambda: \mathbb{R} \times \mathbb{R} \rightarrow \mathbb{T}$ defined by $\lambda(x, y)=\chi_{1}(x y)$, where $\chi_{1}: \mathbb{R} \rightarrow \mathbb{T}$ is the character determined by the canonical quotient map $\mathbb{R} \rightarrow \mathbb{T}=\mathbb{R} / \mathbb{Z}$. Indeed, for every $y \in \mathbb{R}$ the map $\chi_{y}: \mathbb{R} \rightarrow \mathbb{T}$ defined by $x \mapsto \lambda(x, y)$ is an element of $\widehat{\mathbb{R}}$. Hence the second copy $\{0\} \times \mathbb{R}$ of $\mathbb{R}$ in $\mathbb{R} \times \mathbb{R}$ can be identified with $\widehat{\mathbb{R}}$. On the other hand, every element $x \in \mathbb{R}$ gives a continuous character $\mathbb{R} \rightarrow \mathbb{T}$ defined by $y \mapsto \lambda(x, y)$, so can be considered as the element $\omega_{\mathbb{R}}(x)$ of $\widehat{\mathbb{R}}$. We have seen that every $\xi \in \widehat{\widehat{\mathbb{R}}}$ has this form. This means that $\omega_{\mathbb{R}}$ is surjective. Since continuity of $\omega_{\mathbb{R}}$, as well as local compactness of $\widehat{\widehat{\mathbb{R}}}$ are already established, $\omega_{\mathbb{R}}$ is a topological isomorphism by the open mapping theorem.

For a subset $X$ of $G$ the annihilator of $X$ in $\widehat{G}$ is $A_{\widehat{G}}(X)=\{\chi \in \widehat{G}: \chi(A)=\{0\}\}$ and for a subset $Y$ of $\widehat{G}$ the annihilator of $Y$ in $G$ is $A_{G}(Y)=\{x \in G: \chi(x)=0$ for every $x \in Y\}$. When no confusion is possible we shall omit the subscripts $\widehat{G}_{G}$ and ${ }_{G}$.

The next lemma will help us in computing the dual of a subgroup and a quotient group.

Lemma 7.27. Let $G$ be a locally compact abelian group. If $M$ is a subset of $G$, then $A_{\widehat{G}}(M)$ is a closed subgroup of $\widehat{G}$.

Proof. It suffices to note that

$$
A_{\widehat{G}}(M)=\bigcap_{x \in M}\{\chi \in \widehat{G}: \chi(x)\}=\bigcap\left\{\operatorname{ker} \omega_{G}(x): x \in M\right\},
$$

where each $\operatorname{ker} \omega(x)$ is a closed subgroup of $\widehat{G}$.

Call a continuous homomorphism $f: G \rightarrow H$ of topological groups proper if $f: G \rightarrow f(G)$ is open, whenever $f(G)$ carries the topology inherited from $H$. In particular, a surjective continuous homomorphism is proper iff it is open.

A short sequence $0 \rightarrow G_{1} \stackrel{f}{\rightarrow} G \stackrel{h}{\rightarrow} G_{2} \rightarrow 0$ in $\mathcal{L}$, where $f$ and $h$ are continuous homomorphisms, is exact if $f$ is injective, $h$ is surjective and $\operatorname{im} f=\operatorname{ker} h$. It is proper if $f$ and $h$ are proper.

Lemma 7.28. Let $G$ be a locally compact abelian group, $H$ a subgroup of $G$ and $i: H \rightarrow G$ the canonical inclusion of $H$ in $G$. Then

(a) $\widehat{i}: \widehat{G} \rightarrow \widehat{H}$ is surjective if $H$ is dense or open or compact;

(b) $\hat{i}$ is injective if and only if $H$ is dense in $G$;

(c) if $H$ is closed and $\pi: G \rightarrow G / H$ is the canonical projection, then the sequence

$$
0 \rightarrow \widehat{G / H} \stackrel{\widehat{\pi}}{\rightarrow} \widehat{G} \stackrel{\widehat{i}}{\rightarrow} \widehat{H}
$$

is exact, $\widehat{\pi}$ is proper and $\operatorname{im} \widehat{\pi}=A_{\widehat{G}}(H)$. If $H$ is open or compact, then $\widehat{i}$ is open and surjective.

Proof. (a) Note that $\widehat{i}$ is surjective if and only if for every $\chi \in \widehat{H}$ there exists $\xi \in \widehat{G}$ such that $\xi \Gamma_{H}=\chi$. If $H$ is compact apply Corollary 6.20. Otherwise Lemma 7.16 applies.

(b) If $H$ is dense, then $\widehat{i}$ is injective by Lemma 7.16. Conversely, assume that $\bar{H}$ is a proper subgroup of $G$ and let $q: G \rightarrow G / \bar{H}$ be the canonical projection. By Theorem 6.19 there exists $\chi \in \widehat{G / \bar{H}}$ not identically zero. Then $\xi=\chi \circ q \in \widehat{G}$ is non-zero and satisfies $\xi(H)=\{0\}$, i.e., $\widehat{i}(\xi)=0$. This implies that $\widehat{i}$ is not injective.

(c) According to Lemma $7.16 \widehat{\pi}$ is a monomorphism, since $\pi$ is surjective. We have that $\widehat{i} \circ \widehat{\pi}=\widehat{\pi \circ} i=0$. If $\xi \in \operatorname{ker} \widehat{i}=\{\chi \in \widehat{G}: \chi(H)=\{0\}\}$, then $\xi(H)=\{0\}$. So there exists $\xi_{1} \in \widehat{G / H}$ such that $\xi=\xi_{1} \circ \pi$ (i.e. $\left.\xi=\widehat{\pi}\left(\xi_{1}\right)\right)$ and we can conclude that $\operatorname{ker} \widehat{i}=\operatorname{im} \widehat{\pi}$. So the sequence is exact and $\operatorname{im} \widehat{\pi}=\operatorname{ker} \widehat{i}=A_{\widehat{G}}(H)$.

To show that $\widehat{\pi}$ is proper it suffices to apply Lemma 7.16 .

If $H$ is open or compact, (a) implies that $\widehat{i}$ is surjective. It remains to show that $\widehat{i}$ is open. If $H$ is compact then $\widehat{H}$ is discrete by Example 7.1(2), so $\widehat{i}$ is obviously open. If $H$ is open, let $K$ be a compact neighborhood of 0 in $G$ such that $K \subseteq H$. Then $W=W_{\widehat{G}}\left(K, \overline{\Lambda_{4}}\right)$ is a compact neighborhood of 0 in $\widehat{G}$. Since $\widehat{i}$ is surjective, $V=\widehat{i}(W)=W_{\widehat{H}}\left(K, \overline{\Lambda_{4}}\right)$ is a neighborhood of 0 in $\widehat{H}$. Now $M=\langle W\rangle$ and $M_{1}=\langle V\rangle$ are open compactly generated subgroups respectively of $\widehat{G}$ and $\widehat{H}$, and $\widehat{i}(M)=M_{1}$. Since $M$ is $\sigma$-compact by Lemma 4.12 , we can apply Theorem 4.9 to the continuous surjective homomorphism $\widehat{i} \uparrow_{M}: M \rightarrow M_{1}$ and so also $\widehat{i}$ is open. 
The lemma gives these immediate corollaries:

Corollary 7.29. Let $G$ be a locally compact abelian group and let $H$ be a closed subgroup of $G$. Then $\widehat{G / H} \cong$ $A_{\widehat{G}}(H)$. Moreover, if $H$ is open or compact, then $\widehat{H} \cong \widehat{G} / A_{\widehat{G}}(H)$.

The next corollary says that the duality functor preserves proper exactness for some sequences.

Corollary 7.30. If the sequence $0 \rightarrow G_{1} \stackrel{f}{\rightarrow} G \stackrel{h}{\rightarrow} G_{2} \rightarrow 0$ in $\mathcal{L}$ is proper exact, with $G_{1}$ compact or $G_{2}$ discrete, then $0 \rightarrow \widehat{G_{2}} \stackrel{\widehat{h}}{\rightarrow} \widehat{G} \stackrel{\widehat{f}}{\rightarrow} \widehat{G_{1}} \rightarrow 0$ is proper exact with the same property.

Now we can prove prove the Pontryagin-van Kampen duality theorem, namely $\omega$ is a natural equivalence from $i d_{\mathcal{L}}$ to $\widehat{\widehat{L}}: \mathcal{L} \rightarrow \mathcal{L}$

Theorem 7.31. If $G$ is a locally compact abelian group, then $\omega_{G}$ is a topological isomorphism of $G$ onto $\widehat{\widehat{G}}$.

Proof. We know by Proposition 7.23 that $\omega$ is a natural transformation from $i d_{\mathcal{L}}$ to $\widehat{\widehat{\varkappa}}: \mathcal{L} \rightarrow \mathcal{L}$. Our plan is to chase the given locally compact abelian group $G$ into an appropriately chosen proper exact sequence

$$
0 \rightarrow G_{1} \stackrel{f}{\rightarrow} G \stackrel{h}{\rightarrow} G_{2} \rightarrow 0
$$

in $\mathcal{L}$, with $G_{1}$ compact or $G_{2}$ discrete, such that $G_{1}$ and $G_{2}$ satisfy the duality theorem. By Corollary 7.30 the sequences

$$
0 \rightarrow \widehat{G}_{2} \stackrel{\widehat{h}}{\rightarrow} \widehat{G} \stackrel{\widehat{f}}{\rightarrow} \widehat{G}_{2} \rightarrow 0 \quad \text { and } \quad 0 \rightarrow \widehat{\widehat{G}}_{1} \stackrel{\widehat{\widehat{f}}}{\rightarrow} \widehat{\widehat{G}} \stackrel{\widehat{\hat{h}}}{\rightarrow} \widehat{\widehat{G}}_{2} \rightarrow 0
$$

are proper exact. According to Proposition 7.23 the following diagram commutes:

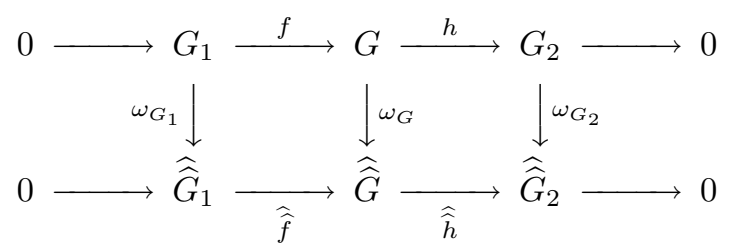

According to Theorem $6.19, \omega_{G_{1}}, \omega_{G}, \omega_{G_{2}}$ are injective. Moreover, $\omega_{G_{1}}$ and $\omega_{G_{2}}$ are surjective by our choice of $G_{1}$ and $G_{2}$. Then $\omega_{G}$ must be surjective too. (Indeed, if $x \in \operatorname{ker} \widehat{\widehat{h}}$, then there exists $y \in \omega_{G}(G)$ with $\widehat{\widehat{h}}(x)=\widehat{\widehat{h}}(y)$, because $\widehat{\widehat{h}}\left(\omega_{G}(G)\right)=\widehat{\widehat{G}}_{2}$. Now $y-x \in \operatorname{ker} \widehat{\widehat{h}} \subseteq \omega_{G}(G)$ and so $x \in y+\omega_{G}(G)=\omega_{G}(G)$.)

If $G$ is locally compact abelian and compactly generated, by Proposition 6.18 we can choose $G_{1}$ compact and $G_{2}$ elementary locally compact abelian. Then $G_{1}$ and $G_{2}$ satisfy the duality theorem by Theorems 7.25 and 7.26 , hence $\omega_{G}$ is surjective. Since $\omega_{G}$ is a continuous isomorphism and $G$ is $\sigma$-compact, we conclude with Theorem 4.9 that $\omega_{G}$ is a topological isomorphism.

In the general case of locally compact abelian group $G$, we can take an open compactly generated subgroup $G_{1}$ of $G$. This will produce a proper exact sequence $0 \rightarrow G_{1} \stackrel{f}{\rightarrow} G \stackrel{h}{\longrightarrow} G_{2} \rightarrow 0$ with $G_{1}$ compactly generated and $G_{2} \cong G / G_{1}$ discrete. By the previous case $\omega_{G_{1}}$ is a topological isomorphism and $\omega_{G_{2}}$ is an isomorphism thanks to Theorem 7.25. Therefore $\omega_{G}$ is a continuous isomorphism.

Moreover $\omega_{G} \uparrow_{f\left(G_{1}\right)}: f\left(G_{1}\right) \rightarrow \widehat{\widehat{f}}\left(\widehat{\widehat{G}}_{1}\right)$ is a topological isomorphism (as $\omega_{G_{1}}, f: G_{1} \rightarrow f\left(G_{1}\right)$ and $\widehat{\widehat{f}}: \widehat{\widehat{G}}_{1} \rightarrow$ $\widehat{\widehat{f}}\left(\widehat{\widehat{G}}_{1}\right)$ are topological isomorphisms) and $f\left(G_{1}\right)$ and $\widehat{\widehat{f}}\left(\widehat{\widehat{G}}_{1}\right)$ are open subgroups respectively of $G$ and $\widehat{\widehat{G}}$. Thus $\omega_{G}$ is a topological isomorphism.

Our last aim is to prove that the annihilators define an inclusion-inverting bijection between the family of all closed subgroups of a locally compact group $G$ and the family of all closed subgroups of $\widehat{G}$. We use that fact that one can identify $G$ and $\widehat{\widehat{G}}$ by the topological isomorphism $\omega_{G}$. In more precise terms:

Exercise 7.32. Let $G$ be a locally compact abelian group and $Y$ be a subset of $\widehat{G}$. Then $A_{\widehat{G}}(Y)=\omega_{G}\left(A_{G}(Y)\right)$.

Lemma 7.33. Let $G$ be a locally compact abelian group and $H$ a closed subgroup of $G$. If $a \in G \backslash H$ then there exists $\chi \in A(H)$ such that $\chi(x) \neq 0$.

Proof. Let $\rho: \widehat{G / H} \rightarrow A(H)$ be the topological isomorphism of Corollary 7.29. By Theorem 6.19 there exists $\psi \in \widehat{G / H}$ such that $\psi(a+H) \neq 0$. Therefore $\chi=\rho(\psi) \in A(H)$ and $\chi(a)=\rho(\psi)(a)=\psi(a+H) \neq 0$. 
Corollary 7.34. If $G$ is a locally compact abelian group and $H$ a closed subgroup of $G$, then

$$
\left.H=A_{G}\left(A_{\widehat{G}}(H)\right)\right)=\omega_{G}^{-1}\left(A_{\widehat{G}}\left(A_{\widehat{G}}(H)\right)\right) .
$$

Proof. The first equality follows immediately from the above lemma.

The last equality follows from the equality $\left.H=A_{G}\left(A_{\widehat{G}}(H)\right)\right)$ and Exercise 7.32 .

By Lemma 7.29 the equality $\left.H=A_{G}\left(A_{\widehat{G}}(H)\right)\right)$ holds if and only if $H$ is a closed subgroup of $G$.

Proposition 7.35. Let $G$ be a locally compact abelian group and $H$ a closed subgroup of $G$. Then $\widehat{H} \cong \widehat{G} / A(H)$. Proof. Since $H=\omega_{G}^{-1}\left(A_{\widehat{G}}\left(A_{\widehat{G}}(H)\right)\right)$ by Lemma 7.34 we have a topological isomorphism $\phi$ from $H$ to $\widehat{G} / A(H)$ given by $\phi(h)(\alpha+A(H))=\alpha(h)$ for every $h \in H$ and $\alpha \in \widehat{G}$. This gives rise to another topological isomorphism $\widehat{\phi}: \widehat{\bar{G} / A(H)} \rightarrow \widehat{H}$. By Pontryagin's duality theorem $7.31 \omega_{\widehat{G} / A(H)}$ is a topological isomorphism from $\widehat{G} / A(H)$ to $\widehat{G / A(H)}$. The composition gives the desired isomorphism.

Finally, let us resume for reader's benefit some of the most relevant points of Pontryagin-van Kampen duality theorem established so far:

Theorem 7.36. Let $G$ be a locally compact abelian group. Then $\widehat{G}$ is a locally compact abelian group and:

(a) the correspondence $H \mapsto A_{\widehat{G}}(H), N \mapsto A_{G}(N)$, where $H$ is a closed subgroup of $G$ and $N$ is a closed subgroup of $\widehat{G}$, defines an order-inverting bijection between the family of all closed subgroups of $G$ and the family of all closed subgroups of $\widehat{G}$;

(b) for every closed subgroup $H$ of $G$ the dual group $\widehat{H}$ is isomorphic to $\widehat{G} / A(H)$, while $A(H)$ is isomorphic to the dual $\widehat{G / H}$;

(c) $\omega_{G}: G \rightarrow \widehat{\widehat{G}}$ is a topological isomorphism;

(d) $G$ is compact (resp., discrete) if and only if $\widehat{G}$ is discrete (resp., compact);

Proof. The first sentence is proved in Theorem 7.2. (a) is Corollary 7.34 while (b) is Proposition 7.35. (c) is Theorem 7.31. To prove (d) apply Theorem 7.31 and Lemma 7.1.

Using the full power of the duality theorem one can prove the following structure theorem on compactly generated locally compact abelian groups.

Theorem 7.37. Let $G$ be a locally compact compactly generated abelian group. Prove that $G \cong \mathbb{R}^{n} \times \mathbb{Z}^{m} \times K$, where $n, m \in \mathbb{N}$ and $K$ is a compact abelian group.

Proof. According to Theorem 6.18 there exists a compact subgroup $K$ of $G$ such that $G / K$ is an elementary locally compact abelian group. Taking a bigger compact subgroup one can get the quotient $G / K$ to be of the form $\mathbb{R}^{n} \times \mathbb{Z}^{m}$ for some $n, m \in \mathbb{N}$. Now the dual group $\widehat{G}$ has an open subgroup $A(K) \cong \widehat{G / K} \cong \mathbb{R}^{n} \times \mathbb{T}^{m}$. Since this subgroup is divisible, one has $\widehat{G} \cong \mathbb{R}^{n} \times \mathbb{T}^{m} \times D$, where $D \cong \widehat{G} / A(K)$ is discrete and $D \cong \widehat{K}$. Taking duals gives $G \cong \widehat{\widehat{G}} \cong \mathbb{R}^{n} \times \mathbb{Z}^{m} \times K$.

Making sharp use of the annihilators one can prove the structure theorem on locally compact abelian groups (see $[67,36]$ for a proof).

Theorem 7.38. Let $G$ be a locally compact abelian group. Then $G \cong \mathbb{R}^{n} \times G_{0}$, where $G_{0}$ is a closed subgroup of $G$ containing an open compact subgroup $K$.

As a corollary one can prove:

Corollary 7.39. Every locally compact abelian group is isomorphic to a subgroup of a group of the form $\mathbb{R}^{n} \times D \times K$, where $n \in \mathbb{N}, D$ is a discrete abelian group and $K$ is a compact abelian group.

Exercise 7.40. Let $G$ be a locally compact abelian group. Prove that for $\chi_{1}, \ldots, \chi_{n} \in \widehat{G}$ and $\delta>0$ one has

$$
U_{G}\left(\chi_{1}, \ldots, \chi_{n} ; \delta\right)=\omega_{G}^{-1}\left(W_{\widehat{G}}\left(\left\{\chi_{1}, \ldots, \chi_{n}\right\}, U\right)\right.
$$

where $U$ is the neighborhood of 0 in $\mathbb{T} \cong \mathbb{S}$ determined by $|\operatorname{Arg} z|<\delta$. 
Exercise 7.41. Let $G$ be a compact connected abelian group. Prove that $t(G)$ is dense in $G$ iff $\widehat{G}$ is reduced. Deduce that every compact connected abelian group $G$ has the form $G \cong G_{1} \times \mathbb{Q}^{\alpha}$ for some cardinal $\alpha$, where the compact subgroup $G_{1}$ coincides with the closure of the subgroup $t(G)$ of $G$.

(Hint. Note first that $\widehat{G}$ is torsion-free. Deduce that $\widehat{G}$ is reduced iff $\bigcap_{n=1}^{\infty} n \widehat{G}=0$. Show that this equality is equivalent to density of $t(G)=\bigcup_{n=1}^{\infty} G[n]$ in $G$. To prove the second assertion consider the torsion-free dual $\widehat{G}$ and its decomposition $\widehat{G}=d(\widehat{G}) \times R$, where $R$ is a reduced subgroup of $\widehat{G}$. Now apply the first part and the isomorphism $G \cong \widehat{\widehat{G}}$.)

Exercise 7.42. Give example of a reduced abelian group $G$ such that $\bigcap_{n=1}^{\infty} n G \neq 0$.

(Hint. Fix a prime number $p$ and take an appropriate quotient of the group $\bigoplus_{n=1}^{\infty} \mathbb{Z}\left(p^{n}\right)$.

\section{Appendix}

\subsection{Uniqueness of Pontryagin-van Kampen duality}

For topological abelian groups $G, H$ denote by $\operatorname{Chom}(G, H)$ the group of all continuous homomorphisms $G \rightarrow H$ equipped with the compact-open topology. It was pointed out already by Pontryagin that the group $\mathbb{T}$ is the unique locally compact group $L$ with the property $\operatorname{Chom}(\operatorname{Chom}(\mathbb{T}, L), L) \cong \mathbb{T}$ (note that this is much weaker than asking $C h o m(-, L)$ to define a duality of $\mathcal{L})$. Much later Roeder [91] proved that Pontryagin-van Kampen dualityis the unique functorial duality of $\mathcal{L}$, i.e., the unique involutive contravariant endofunctor $\mathcal{L} \rightarrow \mathcal{L}$. Several years later Prodanov [85] rediscovered this result in the following much more general setting. Let $R$ be a locally compact commutative ring and $\mathcal{L}_{R}$ be the category of locally compact topological $R$-modules. A functorial duality $\#: \mathcal{L}_{R} \rightarrow \mathcal{L}_{R}$ is a contravariant functor such that \#. \# is naturally equivalent to the identity of $\mathcal{L}_{R}$ and for each morphism $f: M \rightarrow N$ in $\mathcal{L}_{R}$ and $r \in R(r f)^{\#}=r f^{\#}$ (where, as usual, $r f$ is the morphism $M \rightarrow N$ defined by $(r f)(x)=r f(x))$. It is easy to see that the restriction of the Pontryagin-van Kampen duality functor on $\mathcal{L}_{R}$ is a functorial duality, since the Pontryagin-van Kampen dual $\widehat{M}$ of an $M \in \mathcal{L}_{R}$ has a natural structure of an $R$-module. So there is always a functorial duality in $\mathcal{L}_{R}$. This stimulated Prodanov to raise the question how many functorial dualities can carry $\mathcal{L}_{R}$ and extend this question to other well known dualities and adjunctions, such as Stone duality ${ }^{13}$, the spectrum of a commutative rings [86], etc. at his Seminar on dualities (Sofia University, 1979/83). Uniqueness of the functorial duality was obtained by L. Stoyanov [93] in the case of a compact commutative ring $R$. In 1988 Gregorio [56] extended this result to the general case of compact (not necessarily commutative) ring $R$ (here left and right $R$-modules should be distinguished, so that the dualities are no more endofunctors). Later Gregorio jointly with Orsatti [58] offered another approach to this phenomenon.

Surprisingly the case of a discrete ring $R$ turned out to be more complicated. For each functorial duality \# $: \mathcal{L}_{R} \rightarrow \mathcal{L}_{R}$ the module $T=R^{\#}$ (the torus of the duality \#) is compact and for every $X \in \mathcal{L}_{R}$ the module $\Delta_{T}(X):=\operatorname{Chom}_{R}(X, T)$ of all continuous $R$-module homomorphisms $X \rightarrow T$, equipped with the compact-open topology, is algebraically isomorphic to $X^{\#}$. The duality \# is called continuous if for each $X$ this isomorphism is also topological, otherwise ${ }^{\#}$ is discontinuous. Clearly, continuous dualities are classified by their tori, which in turn can be classified by means of the Picard group $\operatorname{Pic}(R)$ of $R$. In particular, the unique continuous functorial duality on $\mathcal{L}_{R}$ is the Pontryagin-van Kampen duality if and only if $\operatorname{Pic}(R)=0([29$, Theorem 5.17]). Prodanov [85] (see also [36, §3.4]) proved that every functorial duality on $\mathcal{L}=\mathcal{L}_{\mathbb{Z}}$ is continuous, which in view of $\operatorname{Pic}(\mathbb{Z})=0$ gives another proof of Roeder's theorem of uniqueness. Continuous dualities were studied in the non-commutative context by Gregorio [57]. While the Picard group provides a good tool to measure the failure of uniqueness for continuous dualities, there is still no efficient way to capture it for discontinuous ones. The first example of a discontinuous duality was given in [29, Theorem 11.1]. Discontinuous dualities of $\mathcal{L}_{\mathbb{Q}}$ and its subcategories are discussed in [34]. It was conjectured by Prodanov that in case $R$ is an algebraic number ring uniqueness of dualities is available if and only if $R$ is a principal ideal domain. This conjecture was proved to be true for real algebraic number rings, but Prodanov's conjecture was shown to fail in case $R$ is an order in an imaginary quadratic number field [25].

We will not touch other well-known dualities for module categories such as Morita duality (see [76]) or more general setting of dualities of (representable dualities, adjunctions rather than involutions, etc. [40], [41] and $[83])$.

\footnotetext{
${ }^{13}$ his conjecture that the Stone duality is the unique functorial duality between compact totally disconnected Hausdorff spaces and Boolean algebras was proved to be true by Dimov [39].
} 


\subsection{Non-abelian or non-locally compact groups}

The Pontryagin-van Kampen duality theorem was extended to some non-locally compact abelian topological groups (e.g., infinite powers of the reals, the underlying additive groups of certain linear topological spaces, etc.). A characterization of the abelian topological groups admitting duality were proposed by Venkatamaran [95] and Kye [73], but they contained flaws. These gaps were removed in the recent paper of Hernández [63]. An important class of abelian groups (nuclear groups) were introduced and studied in the monograph [6] (see also [5]) in relation to the duality theorem. Further reference can be found also in [21, 51, 65]

We do not discuss here non-commutative versions of duality for locally compact groups. The difficulties arise already in the compact case - there is no appropriate (or at least, comfortable) structure on the set of irreducible unitary representations of a compact non-abelian group. The reader is referred to [67] for a historical panorama of this trend (Tanaka-KeIn duality, etc.). In the locally compact case one should see the pioneering paper of H. Chu [22], as well as the monograph of Heyer [68] (see also [69]). In the recent survey of Galindo, Hernández, and Wu [53] the reader can find the last achievements in this field (see also [64]).

\subsection{Relations to the topological theory of topological groups}

The Pontryagin-van Kampen dual of a compact abelian group $K$ carries a lot of useful information about the topology of $H$. For example,

- $w(K)=|\widehat{K}|$

- $d(K)=\log |\widehat{K}|=\min \left\{\kappa: 2^{\kappa} \geq|\widehat{K}|\right\}$,

- $K$ is connected iff $\widehat{K}$ is torsion-free,

- $K$ is totally connected iff $\widehat{K}$ is torsion,

- $c(K)=A(t(\widehat{K}))$, where $t(\widehat{K})$ is the torsion subgroup of $\widehat{K}$,

- $\operatorname{dim} K=r_{0}(\widehat{K})$,

- $H^{1}(K, \mathbb{Z}) \cong \widehat{K}$ if $K$ is connected (here $H^{1}(K, \mathbb{Z})$ denotes the first cohomology group),

- for two compact connected abelian groups $K_{1}$ and $K_{2}$ the following are equivalent: (i) $K_{1}$ and $K_{2}$ are homotopically equivalent as topological spaces; (ii) $K_{1}$ and $K_{2}$ are homeomorphic as topological spaces; (iii) $\widehat{K_{1}} \cong \widehat{K_{2}} ;$ (iv) $K_{1} \cong K_{2}$ as topological groups.

The first equality can be generalized to $w(K)=w(\widehat{K})$ for all locally compact abelian groups $K$.

The Pontryagin-van Kampen duality can be used to easily build the Bohr compactification bG of a locally compact abelian group $G$ (this is the reflection of $G$ into the subcategory of compact abelian groups). In the case when $G$ is discrete, $b G$ is simply the completion of $G^{\#}$, the group $G$ equipped with its Bohr topology. One can prove that $b G \cong \widehat{G}_{d}$, where $\widehat{G}_{d}$ denotes the group $\widehat{G}$ equipped with the discrete topology. For a comment on the non-abelian case see $[28,53]$.

Many nice properties of $\mathbb{Z}^{\#}$ can be found in Kunnen and Rudin [72]. For a fast growing sequence $\left(a_{n}\right)$ in $\mathbb{Z}^{\#}$ the range is a closed discrete set of $\mathbb{Z}^{\#}$ (see [53] for further properties of the lacunary sets in $\mathbb{Z}^{\#}$ ), whereas for a polynomial function $n \mapsto a_{n}=P(n)$ the range has no isolated points [72, 44, Theorem 5.4]. Moreover, the range $P(\mathbb{Z})$ is closed when $P(x)=x^{k}$ is a monomial. For quadratic polynomials $P(x)=a x^{2}+b x+c$, $(a, b, c, \in \mathbb{Z}, a \neq 0)$ the situation is already more complicated: the range $P(\mathbb{Z})$ is closed iff there is at most one prime that divides $a$, but does not divide $b[72,44$, Theorem 5.6]. This leaves open the general question [26, Problem 954].

Problem 8.1. Characterize the polynomials $P(x) \in \mathbb{Z}[x]$ such that $P(\mathbb{Z})$ is closed in $Z^{\#}$.

\subsection{Relations to dynamical systems}

Among the known facts relating the dynamical systems with the topic of these notes let us mention just two.

- A compact group $G$ admits ergodic translations $T_{a}(x)=a x$ iff $G$ is monothetic. The ergodic rotations $T_{a}$ of $\mathrm{G}$ are precisely those defined by a topological generator a of $\mathrm{G}$.

- A continuous surjective endomorphism $T: K \rightarrow K$ of a compact abelian group is ergodic iff the dual $\widehat{T}: \widehat{K} \rightarrow \widehat{K}$ has no periodic points except $x=0$.

The Pontryagin-van Kampen duality has an important impact also on the computation of the entropy of endomorphisms of (topological) abelian groups. Adler, Konheim, and McAndrew introduced the notion of topological entropy of continuous self-maps of compact topological spaces in the pioneering paper [1]. In 1975 Weiss [98] developed the definition of entropy for endomorphisms of abelian groups briefly sketched in [1]. He 
called it "algebraic entropy", and gave detailed proofs of its basic properties. His main result was that the topological entropy of a continuous endomorphism $\phi$ of a profinite abelian group coincides with the algebraic entropy of the adjoint map $\widehat{\phi}$ of $\phi$ (note that pro-finite abelian groups are precisely the Pontryagin duals of the torsion abelian groups).

In 1979 Peters [82] extended Weiss's definition of entropy for automorphisms of a discrete abelian group $G$. He generalized Weiss's main result to metrizable compact abelian groups, relating again the opological entropy of a continuous automorphism of such a group $G$ to the entropy of the adjoint automorphism of the dual group $\widehat{G}$. The definition of entropy of automorphisms given by Peters is easily adaptable to endomorphisms of Abelian groups, but it remains unclear whether his theorem can be extended to the computation of the topological entropy of a continuous endomorphism of compact abelian groups. 


\section{References}

[1] R. L. Adler, A. G. Konheim, M. H. McAndrew, Topological entropy, TAMS 114 (1965), 309-319.

[2] D. Alcaraz, D. Dikranjan, M. Sanchis, Infinitude of Bowen's entropy for group endomorphisms, Preprint.

[3] D. L. Armacost, The structure of locally compact Abelian groups, Monographs and Textbooks in Pure and Applied Mathematics, 68, Marcel Dekker, Inc., New York, 1981.

[4] G. Artico, V. Malykhin and U. Marconi, Some large and small sets in topological groups, Math. Pannon. 12 (2001), no. 2, 157-165.

[5] L. Aussenhofer, Contributions to the duality theory of abelian topological groups and to the theory of nuclear groups, Dissertationes Math. (Rozprawy Mat.) 384 (1999), 113 pp.

[6] W. Banaszczyk, Additive subgroups of topological vector spaces, Lecture Notes in Mathematics 1466 , Springer-Verlag, Berlin (1991).

[7] G. Barbieri, D. Dikranjan, C. Milan, H. Weber, Answer to Raczkowski's quests on convergent sequences, Top. Appl. 1321 (2003), 89-101.

[8] G. Barbieri, D. Dikranjan, C. Milan, H. Weber, Convergent sequences in precompact group topologies, Appl. Gen. Topol. 6 (2005), no. 2, 149-169.

[9] G. Barbieri, D. Dikranjan, C. Milan and H. Weber, Topological torsion related to some recursive sequences of integers, Math. Nachrichten, to appear.

[10] G. Baumslag, A.Myasnikov and V. Remeslennikov, Algebraic geometry over groups. I. Algebraic sets and ideal theory, J. Algebra 219 (1999), no. 1, 16-79..

[11] A. Bella and V. Malykhin, On certain subsets of a group, Questions Answers Gen. Topology, 17:183-197, 1999.

[12] A. Bíró, Characterizing sets for subgroups of compact groups, J, Number Theory, to appear.

[13] A. Bíró, J.-M. Deshouillers and V. Sós, Good approximation and characterization of subgroups of $\mathbf{R} / \mathbf{Z}$, Studia Sci. Math. Hungar. 38 (2001), 97-113.

[14] A. Bíró and V. Sós, Strong characterizing sequences in simultaneous Diophantine approximation, J. Number Theory 99 (2003), no. 2, 405-414.

[15] M. Beiglböck, Filters in number theory and combinatorics, PhD Dissertation, Technische Universität Wien, Wien, October 2004.

[16] M. Beiglböck, Strong characterizing sequences of countable subgroups, J. Number Theory, submitted.

[17] M. Beiglböck, C. Steineder and R. Winkler, Sequences and filters of characters characterizing subgroups of compact abelian groups, Topology Appl. 153 (2006), no. 11, 1682-1695.

[18] J. P. Borel, Sous-groupes de $\mathbf{R}$ liés à répartition modulo 1 de suites Ann. Fac. Sci. Toulouse Math. (5) 5 (1983), no. 3-4, 217-235.

[19] J. P. Borel, Sur certains sous-groupes de R liés à la suite des factorielles., Colloq. Math. 62 (1991), no. 1, 21-30.

[20] R. Bryant, The verbal topology of a group, J. Algebra 48 (1977), no. 2, 340-346.

[21] M.J. Chasco, Pontryagin duality for metrizable groups, Arch. Math. (Basel) 70 (1998), no. 1, 22-28.

[22] H. Chu, Compactification and duality of topological groups, Trans. Amer. Math. Soc. 123 (1966), 310-324.

[23] W.W. Comfort and Ross, Topologies induced by groups of characters, Fund. Math. 55 (1964) $283-291$.

[24] M. Cotlar and R. Ricabarra, On the existence of characters in topological groups, Amer. J. Math. 76 (1954), $375-388$. 
[25] D. Dikranjan, Uniqueness of dualities, in: A. Facchini and C. Menini ed., Abelian Groups and Modules, Proc. of the Padova Conference, Padova, Italy, June 23-July 1, 1994, Mathematics and its Applications, vol. 343, Kluwer Academic Publishers, Dordrecht-Boston-London, The Netherlands, 1995, pp. 123-133.

[26] D. Dikranjan, Topologically torsion elements of topological groups, Topology Proc., 26, 2001/2002, pp. 505-532.

[27] D. Dikranjan, Topological groups, in Klaas Pieter Hart, Jun-iti Nagata and Jerry E. Vaughan editors, Encyclopedia of general topology, Edited by Elsevier Science Publishers, B.V., Amsterdam, 2004 . x+526 pp.

[28] D. Dikranjan, Closure operators in topological groups related to von Neumann's kernel, Topology and its Applications, 153 no. 11, (2006), 1930-1955 .

[29] D. Dikranjan and A. Orsatti, On an unpublished manuscript of Ivan Prodanov concerning locally compact modules and their dualities, Comm. Algebra, 17 (1989) 2739-2771.

[30] D. Dikranjan and I. Protasov, Every infinite group can be generated by a P-small subset, Appl. Gen. Topol., Volume 7, No. 2 (2006) 265-268.

[31] D. Dikranjan and R. Di Santo, On Armacost's quest on topologically torsion elements, Communications Algebra, 32 (2004), 133-146.

[32] D. Dikranjan and R. Di Santo, A characterization of the circle group via uniqueness of roots, Topology and its Applications (2007), to appear.

[33] D. Dikranjan and K. Kunen, Characterizing Subgroups of Compact abelian Groups, J. Pure Appl. Algebra, 208 (2006) 285-291.

[34] D. Dikranjan and C. Milan, Dualities of locally compact modules over the rationals, J. Algebra, 256 (2002) pp. $433-466$.

[35] D. Dikranjan, C. Milan and A. Tonolo A characterization of the MAP abelian groups, J. Pure Appl. Algebra, 197 (2005) 23-41.

[36] D. Dikranjan, Iv. Prodanov and L. Stoyanov, Topological Groups: Characters, Dualities and Minimal Group Topologies, Pure and Applied Mathematics, Vol. 130, Marcel Dekker Inc., New York-Basel, 1989.

[37] D. Dikranjan and D. Shakhmatov, Reflection principle characterizing groups in which unconditionally closed sets are algebraic, J. Group Theory, to appear.

[38] D. Dikranjan and Umberto Zannier, On a property of the adele ring of the rationals, Rings, Modules, Algebras and Abelian Groups, Proceedings of the Algebra Conference - Venezia, "Lecture Notes in Pure and Applied Mathematics", vol. 236, Marcel Dekker 2004, pp. 133-137.

[39] G. Dimov, An axiomatic characterization of Stone duality, Serdika 10 (1984) 165-173.

[40] G. Dimov and W. Tholen, Characterization of representable dualities, Proceedings of the International Conference on Categorical Topology, Prague, 1988 (World Scientific, Singapore 1989) 336-357.

[41] G. Dimov and W. Tholen, Group of dualities, Trans. Amer. Math. Soc. 336, n.2 (1993), 901-913.

[42] H.G. Eggleston, Sets of fractional dimensions which occur in some problems of number theory, Proc. London Math. Soc. (2) 54, (1952) 42-93.

[43] R. Ellis and H. B. Keynes, Bohr compactifications and a result of Følner, Israel J. Math. 12 (1972), 314-330.

[44] R. Engelking, General Topology, Heldermann Verlag, Berlin 1989.

[45] E. Følner, Generalization of a theorem of Bogolioúboff to topological abelian groups. With an appendix on Banach mean values in non-abelian groups, Math. Scand. 2 (1954), 518.

[46] L. Fuchs, Infinite Abelian groups, vol. I, Academic Press New York and London, 1973.

[47] L. Fuchs and K. -H. Hofmann, Extensions of compact abelian groups by discrete ones and their duality theory. I, J. Algebra 196 (1997), no. 2, 578-594. 
[48] L. Fuchs and K. -H. Hofmann, Extensions of compact abelian groups by discrete ones and their duality theory. II, in: D. Dikranjan and L. Salce editors, Abelian groups, module theory, and topology (Padua, 1997), 205-225, Lecture Notes in Pure and Appl. Math., 201, Dekker, New York, 1998.

[49] H. Fujita and D. Shakhmatov, A characterization of compactly generated metric groups, Proc. Amer. Math. Soc. 131 (2003), no. 3, 953-961.

[50] H. Fujita and D. Shakhmatov, Topological groups with dense compactly generated subgroups, Appl. Gen. Topol. 3 (2002), no. 1, 85-89.

[51] J. Galindo and S. Hernández, Pontryagin-van Kampen reflexivity for free abelian topological groups, Forum Math. 11 (1999), no. 4, 399-415.

[52] J. Galindo and S. Hernández, Interpolation sets and the Bohr topology of locally compact groups, Adv. Math. 188 (2004), no. 1, 51-68.

[53] J. Galindo, S. Hernández, and T. S. Wu, Recent results and open questions relating Chu duality and Bohr compactifications of locally compact groups, Open problems in topology II (E. Pearl, ed.), Elsevier Science.

[54] E. Glasner, On minimal actions of Polish groups, Topology Appl. 85 (1998), 119-125.

[55] C. C. Graham and K. E. Hare, $\varepsilon$-Kronecker and $I_{0}$-sets in abelian groups. III. Interpolation by measures on small sets, Studia Math. 171 (2005), no. 1, 1532.

[56] E. Gregorio, Dualities over compact rings, Rend. Sem. Mat. Univ. Padova, 80 (1988), 151-174.

[57] E. Gregorio, Tori and continuous dualities, Rend. Accad. Naz. dei XL, Memorie di Mat., 107, vol. XIII, fasc. 13, (1989) 211-221.

[58] E. Gregorio and A. Orsatti, Uniqueness and existence of dualities over compact rings, Tsukuba J. Math. 18, n. 1 (1994) 39-61.

[59] D. Grow, Sidon sets and $I_{0}$-sets, Colloq. Math. 53 (1987), no. 2, 269270.

[60] K. E. Hare and L. T. Ramsey, $I_{0}$-sets in non-abelian groups, Math. Proc. Cambridge Philos. Soc. 135 (2003), no. 1, 81-98.

[61] J. Hart and K. Kunen, Limits in function speces, 151 (2005), no. 1-3, 157-168.

[62] J. Hart and K. Kunen, Limits in compact abelian groups, Topology Appl. 153 (2006), no. 7, 991-1002.

[63] S. Hernández, Pontryagin duality for topological abelian groups, Math. Z. Math. Z. 238 (2001), no. 3, 493-503.

[64] S. Hernández, Extension of continuous functions on product spaces, Bohr compactification and almost periodic functions, Proceedings of the 18th Summer Conference on Topology and its Applications. Topology Proc. 28 (2004), no. 2, 527-540.

[65] S. Hernández and V. Uspenskij, Pontryagin duality for spaces of continuous functions, J. Math. Anal. Appl. 242 (2000), no. 2, 135-144.

[66] G. Hesse, Zur Topologisierbarkeit von Gruppen, Dissertation, Univ. Hannover, Hannover, 1979.

[67] E. Hewitt and K. Ross, Abstract Harmonic Analysis, Volume I, Springer-Verlag, Berlin-GöttingenHeidelberg 1979.

[68] H. Heyer, Dualitïokalkompakter Gruppen, Lecture Notes in Math., vol. 150, Springer, Berlin, 1970.

[69] H. Heyer, Groups with Chu duality, Probability and information theory, II, Lecture Notes in Math., vol. 296, Springer, Berlin, 1973, pp. 181215.

[70] K. H. Hofmann and S. Morris, The structure of compact groups. A primer for the student-a handbook for the expert, de Gruyter Studies in Mathematics, 25. Walter de Gruyter \& Co., Berlin, 1998. xviii+835 pp.

[71] C. Kraaikamp and P. Liardet, Good approximations and continued fractions, Proc. Amer. Math. Soc. 112 (1991), no. 2, 303-309. 
[72] K. Kunen and W. Rudin, Lacunarity and the Bohr topology, Math. Proc. Cambridge Philos. Soc. 126 (1999), no. 1, 117-137.

[73] S.-H. Kye, Pontryagin duality in real linear topological spaces, Chinese J. Math. 12 (1984), no. 2, $129-136$.

[74] G. Larcher, A convergence problem connected with the continued fractions, Proc. Amer. Math. Soc., 103 (1988), no. 3, 718-722.

[75] G. W. Mackey, Harmonic analysis as the exploitation of symmetry-a historical survey, Bull. Amer. Math. Soc. (N.S.) 3 (1980), no. 1, part 1, 543-698.

[76] A. A. Markov, On unconditionally closed sets, In: Topology and Topological Algebra, Translations Series 1, vol. 8, pages 273-304. American Math. Society, 1962. Russian original in: Comptes Rendus Dokl. AN SSSR (N.S.) 44 (1944), 180-181.

[77] K. Morita, Duality for modules and its application to the theory of rings with minimum condition, Sci. Report Tokyo Kyoiku Daigaku, 6 (1958), 83-142.

[78] A. Yu. Olshanskii, A remark on a countable nontopologized group, Vestnik Moskov. Univ. Ser. I Mat. Mekh., No. 3, p. 103 (1980);

[79] A. Orsatti, Introduzione ai groups astratti e topologici, Quaderni dell'Unione Matematica Italiana, Pitagora Ed., Bologna, 1979.

[80] V. Pestov, Forty-plus annotated questions about large topological groups, in: Open Problems in Topology II, Elliott Pearl Editor, Elsevier B.V., March, 2007, pp. 439 - 450.

[81] J. Peters, Entropy on discrete Abelian groups, Adv. Math. 33 (1979), 1-13.

[82] L. Pontryagin, Topological groups, Transl. Russian, Arlen Brown Gordon and Breach Science Publishers, Inc., New York-London-Paris 1966.

[83] H. Porst and W. Tholen, Concrete dualities, Categiry theory at work (Bremen, 1990), Res. Exp. Math. 18, (Heldermann, Berlin, 1991), 111-136.

[84] Iv. Prodanov, Elementary proof of the Peter-Weyl theorem, (Russian) C. R. Acad. Bulgare Sci. 34 (1981), no. $3,315-318$.

[85] Iv. Prodanov, An axiomatic characterization of the Pontryagin duality, Preprint, 1979.

[86] Iv. Prodanov, An abstract approach to the algebraic notion of spectrum, (Russian) Topology (Moscow, 1979). Trudy Mat. Inst. Steklov, 154 (1983), 200-208, Amer. Math. Soc. Transl. Proceedings of the Steklov Institute of Mathematics 1984, Issue

[87] I. V. Protasov and T. Banakh, Ball Structures and Colorings of Graphs and Groups, Matem. Stud. Monogr. Series, Vol 11, Lviv, 2003.

[88] I. V. Protasov and E. G. Zelenyuk, Topologies on abelian groups, Math. USSR Izvestiya 37 (1991), 445-460. Russian original: Izvestia Akad. Nauk SSSR 54 (1990), 1090-1107.

[89] I. Protasov, E. Zelenyuk, Topologies on Groups Determined by Sequences, Math. Stud. Monogr. Ser.,4, 1999.

[90] D. W. Roeder, A characterization of unitary duality, Trans. Amer. Math. Soc. 148 (1970), 129-135.

[91] D. W. Roeder, Functorial characterization of Pontryagin duality, Trans. Amer. Math. Soc. 154 (1971), $151-175$.

[92] S. Shelah, On a problem of Kurosh, Jonsson groups, and applications, in Word Problems II, Ed. by S. I. Adian, W. W. Boone, and G. Higman (North-Holland, Amsterdam, 1980), pp. 373-394.

[93] L. Stoyanov, Dualities over compact commutative rings, Rend. Accad. Naz. delle Sci. detta dei XL, Memorie di Mat. 101, Vol. VII, fasc. 10 (1983), 155-176.

[94] W. A. Veech, The equicontinuous structure relation for minimal Abelian transformation groups, Amer. J. Math. 90 (1968), 723-732. 
[95] R. Venkataraman, A characterization of Pontryagin duality, Math. Z. 149 (1976), no. 2, 109-119.

[96] Yuan Wang (editor), The Goldbach conjecture, Second edition, Series in Pure Mathematics, 4. World Scientific Publishing Co., Inc., River Edge, NJ, 2002.

[97] A. Weil, Basic number theory, Die Grundlagen der mathematischen Wissenshaften in Einzeldarstellungen, Band 144, Springer-Verlag, Berlin Heidelberg New York, 1970.

[98] M. D. Weiss, Algebraic and other entropies of group endomorphisms, Math. system Theory, 8 (1974/75), no. $3,243-248$.

[99] R. Winkler, Ergodic group rotations, Hartman sets and Kronecker sequences, Dedicated to Edmund Hlawka on the occasion of his 85th birthday. Monatsh. Math. 135 (2002), no. 4, 333-343. 